\title{
2016 American Thyroid Association Guidelines for Diagnosis and Management of Hyperthyroidism and other causes of Thyrotoxicosis
}

*Douglas S. Ross ${ }^{1}, * *$ Henry B. Burch ${ }^{2}$, David S. Cooper ${ }^{3}$, M. Carol Greenlee ${ }^{4}$, Peter Laurberg, ${ }^{5}$ Ana Luiza Maia ${ }^{6}$, Scott A. Rivkees ${ }^{7}$, Mary Samuels ${ }^{8}$, Julie Ann Sosa ${ }^{9}$, Marius N. $\operatorname{Stan}^{10}$, and Martin A. Walter ${ }^{11}$

${ }^{1}$ Massachusetts General Hospital, Boston, Massachusetts.

${ }^{2}$ Endocrinology - Metabolic Service, Walter Reed National Military Medical Center, Bethesda, Maryland.

${ }^{3}$ Division of Endocrinology, Diabetes, \& Metabolism, The Johns Hopkins University School of Medicine, Baltimore, Maryland.

${ }^{4}$ Western Slope Endocrinology, Grand Junction, CO.

${ }^{5}$ Departments of Clinical Medicine and Endocrinology, Aalborg University and Aalborg University Hospital, Aalborg, Denmark.

${ }^{6}$ Thyroid Section, Hospital de Clinicas de Porto Alegre, Federal University of Rio Grande do Sul, Porto Alegre, Brazil.

${ }^{7}$ Pediatrics - Chairman's Office, University of Florida College of Medicine, Gainesville, Florida.

${ }^{8}$ Division of Endocrinology, Diabetes and Clinical Nutrition, Oregon Health \& Science University, Portland, Oregon.

${ }^{9}$ Section of Endocrine Surgery, Duke University School of Medicine, Durham, North Carolina.

${ }^{10}$ Division of Endocrinology, Mayo Clinic, Rochester.

${ }^{11}$ Institute of Nuclear Medicine, University Hospital Bern, Switzerland.

*Authorship listed in alphabetical order following the Chairperson.

**One or more of the authors are military service members (or employees of the U.S. Government). The views expressed in this manuscript are those of the authors and do not reflect the official policy of the Department of the Army, the Department of Defense or the United States Government. This work was prepared as part of the service member's official duties. 
47 Background: Thyrotoxicosis has multiple etiologies, manifestations, and potential

48 therapies. Appropriate treatment requires an accurate diagnosis and is influenced by

49 coexisting medical conditions and patient preference. This article describes evidence-

50 based clinical guidelines for the management of thyrotoxicosis that would be useful to

51 generalist and subspecialty physicians and others providing care for patients with this

52 condition.

53 Methods: The American Thyroid Association (ATA) previously co-sponsored guidelines

54 for the management of thyrotoxicosis that were published in 2011. Considerable new

55 literature has been published since 2011, the ATA felt updated evidence-based guidelines

56 were needed, and assembled a task force of expert clinicians who authored this report.

57 The task force examined relevant literature using a systematic PubMed search

58 supplemented with additional published materials. An evidence-based medicine approach

59 that incorporated the knowledge and experience of the panel was used to update the 2011

60 text and recommendations. The strength of the recommendations and the quality of

61 evidence supporting each was rated according to the approach recommended by the

62 Grading of Recommendations, Assessment, Development, and Evaluation Group.

63 Results: Clinical topics addressed include the initial evaluation and management of

64 thyrotoxicosis; management of Graves' hyperthyroidism using radioactive iodine,

65 antithyroid drugs, or surgery; management of toxic multinodular goiter or toxic adenoma

66 using radioactive iodine or surgery; Graves' disease in children, adolescents, or pregnant

67 patients; subclinical hyperthyroidism; hyperthyroidism in patients with Graves'

68 orbitopathy; and management of other miscellaneous causes of thyrotoxicosis. New 
69 paradigms since publication of the 2011 guidelines are presented for the evaluation of the

70 etiology of thyrotoxicosis, the management of Graves' hyperthyroidism with antithyroid

71 drugs, the management of pregnant hyperthyroid patients, and the preparation of patients

72 for thyroid surgery. The sections on less common causes of thyrotoxicosis have been

73 expanded.

74 Conclusions: One hundred twenty-four evidence-based recommendations were

75 developed to aid in the care of patients with thyrotoxicosis and to share what the task

76 force believes is current, rational, and optimal medical practice.

\section{Introduction}

Thyrotoxicosis is a condition having multiple etiologies, manifestations, and

79 potential therapies. The term "thyrotoxicosis" refers to a clinical state that results from

80 inappropriately high thyroid hormone action in tissues generally due to inappropriately

81 high tissue thyroid hormone levels. The term "hyperthyroidism," as used in these

82 guidelines, is a form of thyrotoxicosis due to inappropriately high synthesis and secretion

83 of thyroid hormone(s) by the thyroid. Appropriate treatment of thyrotoxicosis requires an

84 accurate diagnosis. For example, thyroidectomy is an appropriate treatment for some

85 forms of thyrotoxicosis and not for others. Additionally, beta blockers may be used in

86 almost all forms of thyrotoxicosis, whereas antithyroid drugs (ATDs) are useful in only

87 some.

In the United States, the prevalence of hyperthyroidism is approximately $1.2 \%$

89 ( $0.5 \%$ overt and $0.7 \%$ subclinical $)$; the most common causes include Graves' disease 
90 (GD), toxic multinodular goiter (TMNG), and toxic adenoma (TA) (1). Scientific

91 advances relevant to this topic are reported in a wide range of literature, including

92 subspecialty publications in endocrinology, pediatrics, nuclear medicine, and surgery,

93 making it challenging for clinicians to keep abreast of new developments. Although

94 guidelines for the diagnosis and management of patients with thyrotoxicosis were

95 published previously by the American Thyroid Association (ATA) and the American

96 Association of Clinical Endocrinologists (AACE) in 2011, the ATA determined that

97 thyrotoxicosis represents a priority area in need of updated evidence-based practice

98 guidelines.

The target audience for these guidelines includes general and subspecialty

100 physicians and others providing care for patients with thyrotoxicosis. In this document,

101 we outline what we believe is current, rational, and optimal medical practice. It is not the

102 intent of these guidelines to replace clinical judgment, individual decision making, or the

103 wishes of the patient or family. Rather, each recommendation should be evaluated in light

104 of these elements in order that optimal patient care is delivered. In some circumstances, it

105 may be apparent that the level of care required may be best provided in centers where

106 there is specific expertise, and that referral to such centers should be considered.

107 Methods of Development of Evidence-Based Guidelines

108 Administration

109 The ATA Executive Council selected a chairperson to lead the task force and this

110 individual (D.S.R.) identified the other 10 members of the panel in consultation with the 
111 ATA board of directors. Membership on the panel was based on clinical expertise,

112 scholarly approach, and representation of adult and pediatric endocrinology, nuclear

113 medicine, and surgery. The task force included individuals from North America, South

114 America, and Europe. Panel members declared whether they had any potential conflict of

115 interest at the initial meeting of the group and periodically during the course of

116 deliberations. Funding for the guidelines was derived solely from the general funds of the

117 ATA and thus the task force functioned without commercial support.

118 The task force reviewed the 2011 guidelines and published editorials regarding

119 those guidelines, and developed a revised list of the most common causes of

120 thyrotoxicosis and the most important questions that a practitioner might pose when

121 caring for a patient with a particular form of thyrotoxicosis or special clinical condition.

122 One task force member was assigned as the primary writer for each topic. One or more

123 task force members were assigned as secondary writers for each topic, providing their

124 specific expertise and critical review for the primary writer. The relevant literature was

125 reviewed using a systematic PubMed search for primary references and reviews

126 published after the submission of the 2011 guidelines, supplemented with additional

127 published materials found on focused PubMed searches. Recommendations were based

128 on the literature and expert opinion where appropriate. A preliminary document and a

129 series of recommendations concerning all of the topics were generated by each primary

130 writer and then critically reviewed by the task force at large. The panel agreed

131 recommendations would be based on consensus of the panel and that voting would be

132 used if agreement could not be reached. Task force deliberations took place between 
1332014 and 2016 during several lengthy committee meetings, and through electronic

134 communication.

\section{Rating of the recommendations}

136 These guidelines were developed to combine the best scientific evidence with the

137 experience of seasoned clinicians and the pragmatic realities inherent in implementation.

138 The task force elected to rate the recommendations according to the system developed by

139 the Grading of Recommendations, Assessment, Development, and Evaluation Group (3-

140 6). The balance between benefits and risks, quality of evidence, applicability, and

141 certainty of the baseline risk are all considered in judgments about the strength of

142 recommendations (7). Grading the quality of the evidence takes into account study

143 design, study quality, consistency of results, and directness of the evidence. The strength

144 of a recommendation is indicated as a strong recommendation (for or against) that applies

145 to most patients in most circumstances with benefits of action clearly outweighing the

146 risks and burdens (or vice versa), or a weak recommendation or a suggestion that may not

147 be appropriate for every patient, depending on context, patient values, and preferences.

148 The quality of the evidence is indicated as low-quality evidence, moderate-quality

149 evidence, or high-quality evidence, based on consistency of results between studies and

150 study design, limitations, and the directness of the evidence. In several instances, the

151 evidence was insufficient to recommend for or against a test or a treatment, and the task

152 force made a statement labeled "no recommendation." Table 1 describes the criteria to

153 be met for each rating category. Each recommendation is preceded by a description of the 
154 evidence and, in some cases, followed by a remarks section including technical

155 suggestions on issues such as dosing and monitoring.

\section{Presentation of recommendations}

157 The organization of the task force's recommendations is presented in Table 2. The 158 page numbers and the location key can be used to locate specific topics and

159 recommendations. Specific recommendations are presented within boxes in the main

160 body of the text. Location keys can be copied into the Find or Search function in a file or

161 Web page to rapidly navigate to a particular section. A listing of the recommendations

162 without text is provided as Appendix A.

\section{Results}

\section{4 [A] Background}

165 [A1] Causes of thyrotoxicosis

166 In general, thyrotoxicosis can occur if (i) the thyroid is excessively stimulated by

167 trophic factors; (ii) there is constitutive activation of thyroid hormone synthesis and

168 secretion leading to autonomous release of excess thyroid hormone; (iii) thyroid stores of

169 preformed hormone are passively released in excessive amounts owing to autoimmune,

170 infectious, chemical, or mechanical insult; or (iv) there is exposure to extra-thyroidal

171 sources of thyroid hormone, which may be either endogenous (struma ovarii, metastatic

172 differentiated thyroid cancer) or exogenous (factitious thyrotoxicosis). 
174 biochemical severity of the hyperthyroidism, although in reality the disease represents a 175 continuum of overactive thyroid function. Overt hyperthyroidism is defined as a

176 subnormal (usually undetectable) serum thyroid-stimulating hormone (TSH) with

177 elevated serum levels of triiodothyronine $\left(\mathrm{T}_{3}\right)$ and/or free thyroxine estimates (free $\left.\mathrm{T}_{4}\right)$.

178 Subclinical hyperthyroidism is defined as a low or undetectable serum TSH with values

179 within the normal reference range for both $\mathrm{T}_{3}$ and free $\mathrm{T}_{4}$. Both overt and subclinical

180 disease may lead to characteristic signs and symptoms, although subclinical

181 hyperthyroidism is usually considered more mild. Overzealous or suppressive thyroid

182 hormone administration may cause either type of thyrotoxicosis, particularly subclinical

183 thyrotoxicosis. Endogenous overt or subclinical thyrotoxicosis is caused by excess

184 thyroid hormone production and release or by inflammation and release of hormone by 185 the gland.

Endogenous hyperthyroidism is most commonly due to Graves' Disease (GD) or

187 nodular thyroid disease. GD is an autoimmune disorder in which thyrotropin receptor

188 antibodies (TRAb) stimulate the TSH receptor, increasing thyroid hormone production

189 and release. The development of nodular thyroid disease includes growth of established

190 nodules, new nodule formation, and development of autonomy over time (8). In toxic

191 adenomas (TA), autonomous hormone production can be caused by somatic activating

192 mutations of genes regulating thyroid growth and hormone synthesis. Germline

193 mutations in the gene encoding the TSH receptor can cause sporadic or familial

194 nonautoimmune hyperthyroidism associated with a diffuse enlargement of the thyroid

195 gland (9). Autonomous hormone production may progress from subclinical to overt 
196 hyperthyroidism, and the administration of pharmacologic amounts of iodine to such

197 patients may result in iodine-induced hyperthyroidism (10). GD is the most common

198 cause of hyperthyroidism in the United States $(11,12)$. Although toxic nodular goiter is

199 less common than GD, its prevalence increases with age and in the presence of dietary

200 iodine deficiency. Therefore, toxic nodular goiter may actually be more common than

201 GD in older patients, especially in regions of iodine deficiency $(13,14)$. Unlike toxic

202 nodular goiter, which is progressive (unless triggered by excessive iodine intake),

203 remission of mild GD has been reported in up to $30 \%$ of patients without treatment (15).

204 Less common causes of thyrotoxicosis include the entities of painless and

205 subacute thyroiditis, which occur due to inflammation of thyroid tissue with release of

206 preformed hormone into the circulation. Painless thyroiditis caused by lymphocytic

207 inflammation appears to occur with a different frequency depending on the population

208 studied: in Denmark it accounted for only $0.5 \%$ of thyrotoxic patients, it was $6 \%$ of

209 patients in Toronto and $22 \%$ of patients in Wisconsin (16-18).

$210 \quad$ Painless thyroiditis may occur during lithium (19), cytokine (e.g., interferon-

211 alpha) (20), or tyrosine kinase inhibitor therapy (21), and in the postpartum period it is

212 referred to as postpartum thyroiditis (22). A painless destructive thyroiditis (not usually

213 lymphocytic) occurs in 5-10\% of amiodarone-treated patients (23). Subacute thyroiditis

214 is thought to be caused by viral infection and is characterized by fever and thyroid pain

215 (24). 
219 thyroid hormone. $\mathrm{T}_{3}$ binds to a specific nuclear receptor that regulates the expression of 220 many genes. Non-genomic actions of thyroid hormone also regulate numerous important 221 physiologic functions.

Thyroid hormone influences almost every tissue and organ system. It increases

223 tissue thermogenesis and basal metabolic rate (BMR) and reduces serum cholesterol

224 levels and systemic vascular resistance. Some of the most profound effects of increased

225 thyroid hormone levels are on the cardiovascular system (25). Untreated or partially

226 treated thyrotoxicosis is associated with loss of weight, osteoporosis, atrial fibrillation,

227 embolic events, muscle weakness, tremor, neuropsychiatric symptoms and rarely

228 cardiovascular collapse and death $(26,27)$. There is only moderate correlation between

229 the degree of thyroid hormone elevation and clinical signs and symptoms. Symptoms

230 and signs that result from increased adrenergic stimulation include tachycardia and

231 anxiety and may be more pronounced in younger patients and those with larger goiters

232 (28). The signs and symptoms of mild, or subclinical, thyrotoxicosis are similar to those

233 of overt thyrotoxicosis, but differ in magnitude. Measurable changes in basal metabolic

234 rate, cardiovascular hemodynamics, and psychiatric and neuropsychological function can

235 be present in mild thyrotoxicosis (29). 
241 and neuromuscular complications, is essential in formulating an appropriate treatment

242 plan. While it might be anticipated that the severity of thyrotoxic symptoms is

243 proportional to the elevation in the serum levels of free $\mathrm{T}_{4}$ and $\mathrm{T}_{3}$, in one small study of

24425 patients with GD, the Hyperthyroid Symptom Scale did not strongly correlate with

245 free $\mathrm{T}_{4}$ or $\mathrm{T}_{3}$ and was inversely correlated with age (28). The importance of age as a

246 determinant of the prevalence and severity of hyperthyroid symptoms has been recently

247 confirmed (30). Cardiac evaluation may be necessary, especially in the older patient, and

248 may require an echocardiogram, electrocardiogram, Holter monitor, or myocardial

249 perfusion studies (31). This should not postpone therapy of the thyrotoxicosis. In addition

250 to the administration of beta-blockers (31), treatment may be needed for concomitant

251 myocardial ischemia, congestive heart failure, or atrial arrhythmias (25). Anticoagulation

252 may be necessary in patients in atrial fibrillation (32). Goiter size, obstructive symptoms,

253 and the severity of Graves' orbitopathy (GO), the inflammatory disease that develops in

254 the orbit in association with autoimmune thyroid disorders, can be discordant with the

255 degree of hyperthyroidism or hyperthyroid symptoms.

All patients with known or suspected hyperthyroidism should undergo a

257 comprehensive history and physical examination, including measurement of pulse rate,

258 blood pressure, respiratory rate, and body weight. In addition, one should assess thyroid 
259 size, tenderness, symmetry, and nodularity; pulmonary, cardiac, and neuromuscular

260 function $(29,31,33)$; and presence or absence of peripheral edema, eye signs, or pretibial

261 myxedema.

262 [B2] Biochemical evaluation

263 Serum TSH measurement has the highest sensitivity and specificity of any single

264 blood test used in the evaluation of suspected thyrotoxicosis and should be used as an

265 initial screening test (34). However, when thyrotoxicosis is strongly suspected, diagnostic

266 accuracy improves when a serum $\mathrm{TSH}$, free $\mathrm{T}_{4}$, and total $\mathrm{T}_{3}$ are assessed at the initial

267 evaluation. The relationship between free $\mathrm{T}_{4}$ and $\mathrm{TSH}$, when the pituitary-thyroid axis is

268 intact, is an inverse log-linear relationship; therefore, small changes in free $\mathrm{T}_{4}$ result in

269 large changes in serum TSH concentrations. Serum TSH levels are considerably more

270 sensitive than direct thyroid hormone measurements for assessing thyroid hormone

271 excess (35).

In overt hyperthyroidism, serum free $\mathrm{T}_{4}$ and/or $\mathrm{T}_{3}$ are elevated, and serum TSH is

273 subnormal (usually $<0.01 \mathrm{mU} / \mathrm{L}$ in a third generation assay). In mild hyperthyroidism,

274 serum $\mathrm{T}_{4}$ and free $\mathrm{T}_{4}$ can be normal, only serum $\mathrm{T}_{3}$ may be elevated, and serum TSH will

275 be low or undetectable. These laboratory findings have been called " $\mathrm{T}_{3}$-toxicosis" and

276 may represent the earliest stages of hyperthyroidism caused by Graves' disease or an

277 autonomously functioning thyroid nodule. As with $\mathrm{T}_{4}$, total $\mathrm{T}_{3}$ measurements are

278 impacted by protein binding. Assays for estimating free $\mathrm{T}_{3}$ are less widely validated and

279 less robust than those for free $\mathrm{T}_{4}$. Therefore, measurement of total $\mathrm{T}_{3}$ is frequently

280 preferred over free $\mathrm{T}_{3}$ in clinical practice. Subclinical hyperthyroidism is defined as a 
normal serum free $\mathrm{T}_{4}$ and normal total $\mathrm{T}_{3}$ or free $\mathrm{T}_{3}$, with subnormal serum TSH concentration. Laboratory protocols that store sera and automatically retrieve the sample and add on free $\mathrm{T}_{4}$ and total $\mathrm{T}_{3}$ measurements when the initial screening serum TSH concentrations are low, avoid the need for subsequent blood draws. resistance, or spurious assay results due to interfering antibodies, a normal serum

287 TSH level precludes the diagnosis of thyrotoxicosis. The term "euthyroid

288 hyperthyroxinemia' ' has been used to describe a number of entities, primarily thyroid 289 hormone-binding protein disorders, which cause elevated total serum $\mathrm{T}_{4}$ concentrations

290 (and frequently elevated total serum $\mathrm{T}_{3}$ concentrations) in the absence of hyperthyroidism 291 (36). These conditions include elevations in $\mathrm{T}_{4}$ binding globulin (TBG) or transthyretin 292 (TTR) (37); the presence of an abnormal albumin which binds $\mathrm{T}_{4}$ with high capacity 293 (familial dysalbuminemic hyperthyroxinemia); a similarly abnormal TTR; and, rarely, 294 immunoglobulins which directly bind $\mathrm{T}_{4}$ or $\mathrm{T}_{3}$. TBG excess may occur as a hereditary $\mathrm{X}$ 295 linked trait, or be acquired as a result of pregnancy or estrogen administration, hepatitis, 296 acute intermittent porphyuria, or during treatment with 5-flourouracil, perphenazine, or 297 some narcotics. Other causes of euthyroid hyperthyroxinemia include those drugs that 298 inhibit $\mathrm{T}_{4}$ to $\mathrm{T}_{3}$ conversion, such as amiodarone (23) or high-dose propranolol (31), acute 299 psychosis (38), extreme high altitude (39), and amphetamine abuse (40). Estimates of 300 free thyroid hormone concentrations frequently also give erroneous results in these 301 disorders. Spurious free $\mathrm{T}_{4}$ elevations may occur from heterophilic antibodies or in the 302 setting of heparin therapy, due to in vitro activation of lipoprotein lipase and release of 303 free fatty acids that displace $\mathrm{T}_{4}$ from its binding proteins. 
305 be ruled out by repeating the TSH in another assay, measurement of TSH in serial

306 dilution, or direct measurement of human anti-mouse antibodies (HAMA).

308 steptavidin-biotin separation technique $(41,42)$. In immunometric assays, frequently used

309 to measure TSH, excess biotin displaces biotinylated antibodies and causes spuriously

310 low results, while in competitive binding assays, frequently used to measure free $\mathrm{T}_{4}$,

311 excess biotin competes with biotinylated analogue and results in falsely high results.

312 Patients taking high doses of biotin or supplements containing biotin, who have elevated

$313 \mathrm{~T}_{4}$ and suppressed TSH should stop taking biotin and have repeat measurements at least

3142 days later.

315 After excluding euthyroid hyperthyroxinemia, TSH-mediated hyperthyroidism

316 should be considered when thyroid hormone concentrations are elevated and TSH is

317 normal or elevated. A pituitary lesion on MRI and a disproportionately high ratio of the

318 serum level of the alpha-subunit of the pituitary glycoprotein hormones to TSH supports

319 the diagnosis of a TSH-producing pituitary adenoma (43). A family history and genetic

320 testing for mutations in the $\mathrm{T}_{3}$-receptor supports the diagnosis of thyroid hormone

321 resistance (44).

322 [B3] Determination of etiology 
324 The etiology of thyrotoxicosis should be determined. If the diagnosis is not apparent

325 based on the clinical presentation and initial biochemical evaluation, diagnostic testing

326 is indicated and can include, depending on available expertise and resources, (1)

327 measurement of TRAb, (2) determination of the radioactive iodine uptake (RAIU), or

328 (3) measurement of thyroidal blood flow on ultrasonography. An iodine-123 or

329 technetium-99m pertechnetate scan should be obtained when the clinical presentation

330 suggests a toxic adenoma or toxic multinodular goiter. Strong recommendation,

$331 \quad$ moderate-quality evidence.

In a patient with a symmetrically enlarged thyroid, recent onset of orbitopathy,

333 and moderate to severe hyperthyroidism, the diagnosis of GD is likely and further

334 evaluation of hyperthyroidism causation is unnecessary. In a thyrotoxic patient with a

335 non-nodular thyroid and no definite orbitopathy, measurement of TRAb or a radioactive

336 iodine uptake can be used to distinguish GD from other etiologies. In a study using a

337 model of a theoretical population of 100,000 enrollees in a managed care organization in

338 the United States, the use of TRAb measurements to diagnose GD compared to RAIU

339 measurements reduced costs by $47 \%$ and resulted in a $46 \%$ quicker diagnosis (45).

340 A radioactive iodine uptake measures the percentage of administered radioiodine

341 (RAI) that is concentrated into thyroid tissue after a fixed interval, usually 24 hours.

342 Technetium uptake measurements utilize pertechnetate that is trapped by the thyroid, but

343 not organified. A technetium $\left(\mathrm{TcO}_{4}\right)$ uptake measures the percentage of administered

344 technetium that is trapped by the thyroid after a fixed interval, usually 20 minutes. 
346 during pregnancy and usually during lactation (see section [T4]) and distinguishes causes

347 of thyrotoxicosis having elevated or normal uptake over the thyroid gland from those

348 with near-absent uptake (Table 3). Uptake is usually elevated in patients with GD and

349 normal or high in toxic nodular goiter, unless there has been a recent exposure to iodine

350 (e.g., radiocontrast). The RAIU will be near zero in patients with painless, postpartum, or

351 subacute thyroiditis, factitious ingestion of thyroid hormone or recent excess iodine

352 intake. The RAIU may be low after exposure to iodinated contrast in the preceding 1-2

353 months or with ingestion of a diet unusually rich in iodine such as seaweed soup or kelp.

354 However, RAIU is rarely $<\mathbf{1 \%}$ unless the iodine exposure is reoccurring as during

355 treatment with amiodarone. When exposure to excess iodine is suspected (e.g., when the

356 RAIU is lower than expected from the clinical history), assessment of urinary iodine

357 concentration (spot urine iodine adjusted for urine creatinine concentration or a 24-

358 hour urine iodine concentration) may be helpful. The uptake over the neck will also be

359 absent in a patient with struma ovarii, where the abnormal thyroid tissue is located in an

360 ovarian teratoma.

Thyroid scans provide a planar image of the thyroid gland using a gamma camera,

362 to assess potential variability in the concentration of the radioisotope within thyroid

363 tissue. RAI scans may be obtained coincident with the RAIU and technetium scans may

364 be obtained coincident with the technetium uptake. While technetium scans result in a

365 low range of normal uptake and high background activity, total body radiation exposure

366 is less than for ${ }^{123}$ I scans; either type of scan can be useful in determining the etiology of

367 hyperthyroidism in the presence of thyroid nodularity. 
A thyroid scan should be obtained if the clinical presentation suggests a toxic

369 adenoma or toxic multinodular goiter. The pattern of RAIU in GD is diffuse unless there

370 are coexistent nodules or fibrosis. The pattern of uptake in a patient with a single TA

371 generally shows focal uptake in the adenoma with suppressed uptake in the surrounding

372 and contralateral thyroid tissue. The image in TMNG demonstrates multiple areas of

373 focal increased and suppressed uptake. If autonomy is extensive, the image may be

374 difficult to distinguish from that of GD (46). Additionally, GD and non-toxic nodular

375 goiter may coincide resulting in positive TRAb levels and a nodular ultrasound or

376 heterogeneous uptake images (47).

Where expertise is available, ultrasonography with color Doppler flow can

378 distinguish thyroid hyperactivity (increased flow) from destructive thyroiditis (48).

379 Quantitative Doppler evaluation requires careful adjustments to prevent artifacts and

380 measures the peak systolic velocity from intrathyroidal arteries or the inferior thyroidal

381 artery (49). This test may be particularly useful when radioactive iodine is

382 contraindicated, such as during pregnancy or breastfeeding. Doppler flow has also been

383 used to distinguish between subtypes of amiodarone-induced thyrotoxicosis (see Section

384 [V2]), and between GD and destructive thyroiditis (see Section [W2]).

The ratio of total $\mathrm{T}_{3}$ to total $\mathrm{T}_{4}$ can also be useful in assessing the etiology of thyrotoxicosis when scintigraphy is contraindicated. Because a hyperactive gland produces more $T_{3}$ than $T_{4}, T_{3}$ will be elevated above the upper limit of normal more than $T_{4}$ in thyrotoxicosis caused by hyperthyroidism, whereas $T_{4}$ is elevated more than $T_{3}$ in thyrotoxicosis caused by thyroiditis (50); in one study the ratio of total $\mathrm{T}_{3} /$ total $\mathrm{T}_{4}$ 
(ng/mcg) was $>20$ in GD and toxic nodular goiter, and $<20$ in painless or postpartum thyroiditis (51). A high $\mathbf{T}_{\mathbf{4}}$ to $\mathbf{T}_{3}$ ratio may be seen in thyrotoxicosis factitia (from exogenous levothyroxine).

The choice of initial diagnostic testing depends on cost, availability, and local expertise. TRAb is cost-effective because if it is positive it confirms the diagnosis of the most common cause of thyrotoxicosis, but if negative it does not distinguish among other etiologies, and it can be negative in very mild GD. If third generation TRAb assays are not readily available, RAIU is preferred for initial testing.

Diagnostic testing may be influenced by the choice of therapy (see Section [C]). For example, measuring TRAb in a patient with GD who plans on taking methimazole (MMI) with the hope of achieving a remission will provide a baseline measurement for disease activity. Obtaining a RAIU in a patient who prefers radioactive iodine treatment will provide both diagnostic information and facilitate the calculation of the radioactive iodine dose (see Section [D2]).

In most patients, distinction between subacute and painless thyroiditis is not difficult. Subacute thyroiditis is generally painful, the gland is firm to hard on palpation, and the erythrocyte sedimentation rate (ESR) is usually $>50$ and sometimes over 100 $\mathrm{mm} / \mathrm{h}$. Patients with painless thyroiditis presenting within the first year after childbirth (postpartum thyroiditis), often have a personal or family history of autoimmune thyroid disease, and typically have measurable serum concentrations of antithyroid peroxidase antibodies (52). 
419 palpation thyroiditis (following manipulation of the thyroid gland during surgery),

420 whereas its release is suppressed in the setting of exogenous thyroid hormone

421 administration. If not elucidated by the history, factitious ingestion of thyroid hormone

422 can be distinguished from other causes of thyrotoxicosis by a low serum thyroglobulin

423 level, a near-zero RAIU, and a $\mathbf{T}_{3}$ to $\mathbf{T}_{\mathbf{4}}$ ratio $(\mathbf{n g} / \mathbf{m c g})<20$ if due to exogenous

424 levothyroxine. (53). In patients with antithyroglobulin antibodies, which interfere with

425 thyroglobulin measurement, an alternative but not widely available approach is

426 measurement of fecal $\mathrm{T}_{4}(\mathbf{5 4})$; mean values were $1.03 \mathrm{mmol} / \mathrm{g}$ in euthyroid patients,

$427 \quad 1.93 \mathbf{~ n m o l} / \mathrm{g}$ in Graves' hyperthyroidism, and $\mathbf{1 2 - 2 4} \mathbf{~ n m o l} / \mathrm{g}$ in factitious

428 throtoxicosis.

Technical remarks: There are two methods for measuring TRAb (55). Third-

430 generation TBI assays are competition-based assays which measure inhibition of binding

431 of a labeled monoclonal anti-human TSHR antibody (or labeled TSH) to recombinant

432 TSHR. Such assays detect blocking as well as neutral and stimulating immunoglobulins.

433 The newer TSI assays detect increased cAMP production, e.g. from Chinese hamster

434 ovary cells transfected with hTSHR, and are positive in 96\% of patients with GD (56).

435 Older TSI assays were more specific but less sensitive than TBI assays. In the setting of 436 thyrotoxicosis, third generation TBI assays have a sensitivity of $97 \%$ and a specificity of $437 \quad 99 \%(57)$

$438 \quad[B 4]$ Symptomatic management 
440 Beta-adrenergic blockade is recommended in all patients with symptomatic

441 thyrotoxicosis, especially elderly patients and thyrotoxic patients with resting heart

442 rates in excess of $90 \mathrm{bpm}$ or coexistent cardiovascular disease. Strong

443 recommendation, moderate-quality evidence.

444 In a randomized controlled trial of MMI alone versus MMI and a beta-adrenergic

445 blocking agent, after 4 weeks, patients taking beta-adrenergic blockers had lower heart

446 rates, less shortness of breath and fatigue and had improved "physical functioning" on the

$447 \quad$ SF-36 health questionnaire (58).

$448 \quad$ Technical remarks: Since there is not sufficient $\beta-1$ selectivity of the available

449 beta-blockers at the recommended doses, these drugs are generally contraindicated in

450 patients with bronchospastic asthma. In patients with quiescent bronchospastic asthma in

451 whom heart rate control is essential, or in patients with mild obstructive airway disease or

452 symptomatic Raynaud's phenomenon, a relative $\beta-1$ selective agent can be used

453 cautiously, with careful monitoring of pulmonary status (Table 4). Occasionally, very

454 high doses of beta-blockers are required to manage symptoms of thyrotoxicosis and to

455 reduce the heart rate to near the upper limit of normal (31), but most often low to

456 moderate doses (Table 4) give sufficient symptom relief. Oral administration of calcium

457 channel blockers, both verapamil and diltiazem, have been shown to effect rate control in

458 patients who do not tolerate or are not candidates for $\beta$-adrenergic blocking agents.

$460 \quad$ [C] How should overt hyperthyroidism due to GD be managed? 


\section{- RECOMMENDATION 3}

462 Patients with overt Graves' hyperthyroidism should be treated with any of the

463 following modalities: Radioactive iodine therapy, antithyroid drugs, or thyroidectomy.

464 Strong recommendation, moderate-quality evidence.

Once it has been established that the patient is hyperthyroid and the cause is GD,

466 the patient and physician must choose between three effective and relatively safe initial

467 treatment options: RAI therapy, ATDs, or thyroidectomy (59). In the United States, RAI

468 has been the therapy most preferred by physicians but a trend has been present in recent

469 years to increase use of ATDs and reduce the use of RAI. A 2011 survey of clinical

470 endocrinologists showed that $59.7 \%$ of respondents from the United States selected RAI

471 as primary therapy for an uncomplicated case of GD, compared to $69 \%$ in a similar

472 survey performed 20 years earlier (60). In Europe, Latin America, and Japan, there has

473 been a greater physician preference for ATDs (61). The long-term quality of life (QoL)

474 following treatment for GD was found to be the same in patients randomly allocated to

475 one of the three treatment options (62). Currently, there is no scientific evidence to

476 support the recommendation of alternative therapies for the treatment of hyperthyroidism

477 (63).

Technical remarks: Once the diagnosis has been made, the treating physician and

479 patient should discuss each of the treatment options, including the logistics, benefits,

480 expected speed of recovery, drawbacks, potential side effects, and costs (64). This sets

481 the stage for the physician to make recommendations based on best clinical judgment and

482 allows the final decision to incorporate the personal values and preferences of the patient. 
483 The treatment selection should also take into account the local availability and the

484 associated costs. Whenever surgery is selected as treatment one should consider the use

485 of expert high-volume thyroid surgeons with on average lower risk of complications; lack

486 of that expertise should be considered against the known risk of alternative choices.

487 Long term continuous treatment of hyperthyroidism with ATDs may be considered in

488 selected cases $(65,66)$

Clinical situations that favor a particular modality as treatment for Graves'

490 hyperthyroidism (Table 5):

a. RAI therapy: Women planning a pregnancy in the future (in more than 6 months following RAI administration, provided thyroid hormone levels are normal), individuals with comorbidities increasing surgical risk, and patients with previously operated or externally irradiated necks, or lack of access to a highvolume thyroid surgeon, and patients with contraindications to ATD use or failure to achieve euthyroidism during treatment with ATDs. Patients with periodic thyrotoxic hypokalemic paralysis, right heart failure pulmonary hypertension, or congestive heart failure should also be considered good candidates for RAI therapy.

b. ATDs: Patients with high likelihood of remission (patients, especially women, with mild disease, small goiters, and negative or low-titer TRAb); pregnancy; the elderly or others with comorbidities increasing surgical risk or with limited life expectancy; individuals in nursing homes or other care facilities who may have limited longevity and are unable to follow radiation safety regulations; patients 
with previously operated or irradiated necks; patients with lack of access to a high-volume thyroid surgeon; patients with moderate to severe active GO; and patients who need more rapid biochemical disease control. levels are normal ( i.e., possibly before thyroid hormone levels would be normal if RAI were chosen as therapy); symptomatic compression or large goiters ( $\geq 80$ $\mathrm{g}$ ); relatively low uptake of radioactive iodine; when thyroid malignancy is documented or suspected (e.g., suspicious or indeterminate cytology); large thyroid nodules especially if greater than $\mathbf{4} \mathbf{~ c m}$ or if nonfunctioning, or hypofunctioning on iodine-123 or technetium-99m pertechnetate scanning; coexisting hyperparathyroidism requiring surgery; especially if TRAb levels are

517 Contraindications to a particular modality as treatment for Graves'

\section{8 hyperthyroidism:}

a. RAI therapy: Definite contraindications include pregnancy, lactation, coexisting thyroid cancer, or suspicion of thyroid cancer, individuals unable to comply with radiation safety guidelines and used with informed caution in women planning a pregnancy within 4-6 months.

b. ATDs: Definite contraindications to ATD therapy include previous known major adverse reactions to ATDs. 

comorbidity such as cardiopulmonary disease, end-stage cancer, or other debilitating disorders, or lack of access to a high volume thyroid surgeon. Pregnancy is a relative contraindication and surgery should only be used in the circumstance when rapid control of hyperthyroidism is required and antithyroid medications cannot be used. Thyroidectomy is best avoided in the first and third trimesters of pregnancy because of teratogenic effects associated with anesthetic agents and increased risk of fetal loss in the first trimester and increased risk of preterm labor in the third. Optimally, thyroidectomy is performed in the second trimester. Although it is the safest time, it is not without risk $(4.5 \%-5.5 \%$ risk of preterm labor) $(67,68)$. Thyroid surgery in pregnancy is also associated with a higher rate of complications, including hypoparathyroidism and recurrent laryngeal nerve injury (68).

\section{Patient values that may impact choice of therapy:}

a. RAI therapy: Patients choosing RAI therapy as treatment for GD would likely resolution of hyperthyroidism, and potential worsening or development of GO

b. ATDs: Patients choosing ATD as treatment for GD would place relatively higher value on the possibility of remission and the avoidance of lifelong thyroid 

the possibility of disease recurrence. relatively higher value on prompt and definitive control of hyperthyroidism, avoidance of exposure to radioactivity, and the potential side effects of ATDs and a relatively lower value on potential surgical risks, and need for lifelong thyroid hormone replacement.

[D] If RAI therapy is chosen, how should it be accomplished?

559 Because RAI treatment of GD can cause a transient exacerbation of hyperthyroidism,

560 beta-adrenergic blockade should be considered even in asymptomatic patients who are

561 at increased risk for complications due to worsening of hyperthyroidism, i.e., elderly

562 patients and patients with co-morbidities. Weak recommendation, low-quality

$563 \quad$ evidence. 
565 In addition to beta-adrenergic blockade (see Recommendation 2 and 4), pretreatment

566 with MMI prior to RAI therapy for GD should be considered in patients who are at

567 increased risk for complications due to worsening of hyperthyroidism. MMI should be

568 discontinued 2-3 days prior to RAI. Weak recommendation, moderate-quality

$569 \quad$ evidence.

570 - RECOMMENDATION 6

571 In patients who are at increased risk for complications due to worsening of

572 hyperthyroidism, resuming MMI 3-7 days after RAI administration should be

573 considered. Weak recommendation, low-quality evidence.

- RECOMMENDATION 7

575 Medical therapy of any comorbid conditions should be optimized prior to RAI

576 therapy. Strong recommendation, low-quality evidence.

RAI has been used to treat hyperthyroidism for more than seven decades. It is

578 well tolerated and complications are rare, except for those related to orbitopathy (see

579 section [U]). Thyroid storm occurs only rarely following the administration of RAI (70-

580 72). In one study of patients with thyrotoxic cardiac disease treated with RAI as the sole

581 modality, no clinical worsening in any of the cardinal symptoms of thyrotoxicosis was

582 seen (73). However, RAI can induce a short-term increase of thyroid hormone levels

583 (74,75). To prevent a clinical exacerbation of hyperthyroidism, the use of MMI or

584 carbimazole, the latter of which is not marketed in the United States, before and after

585 RAI treatment may be considered in patients with severe hyperthyroidism, the elderly, 
and those with substantial comorbidity that puts them at greater risk for complications of

587 worsening thyrotoxicosis $(75,76)$. The latter includes patients with cardiovascular

588 complications such as atrial fibrillation, heart failure, or pulmonary hypertension and

589 those with renal failure, infection, trauma, poorly controlled diabetes mellitus, and

590 cerebrovascular or pulmonary disease (70). These comorbid conditions should be

591 addressed with standard medical care and the patient rendered medically stable before the

592 administration of RAI if possible. If possible iodinated radiocontrast should be

593 avoided. In addition, beta-adrenergic blocking drugs should be used judiciously in these

594 patients in preparation for RAI therapy (25,77). MMI (75) and carbimazole (78) have

595 shown to reduce thyroid hormone levels after RAI treatment in randomized controlled

596 trials. However, a recent meta-analysis of randomized controlled trials also found that

597 MMI, carbimazole and propylthiouracil reduce the success rate if given in the week

598 before or after RAI treatment (71). Use of higher activities of RAI may offset the reduced

599 effectiveness of RAI therapy following antithyroid medication $(75,76)$.

A special diet is not required before RAI therapy, but nutritional supplements that

601 may contain excess iodine and seaweeds should be avoided for at least 7 days. A low-

602 iodine diet may be useful for those with relatively low RAIU to increase the proportion of

603 RAI trapped.

Technical remarks: Patients that might benefit from adjunctive MMI or

605 carbimazole may be those who are poorly tolerating hyperthyroid symptoms. Such

606 patients frequently have free $\mathrm{T}_{4} 2-3$ times the upper limit of normal. Young and middle

607 aged, otherwise healthy patients who are clinically well-compensated despite significant 
608 biochemical hyperthyroidism can generally receive RAI without pretreatment. If given as 609 pretreatment, MMI and carbimazole should be discontinued before the administration of

610 RAI. Discontinuation of ATDs for 2-3 days prevents a short-term increase of thyroid 611 hormone levels (79), which is found after 6 days $(75,76)$. In elderly patients or in those

612 with underlying cardiovascular disease, resuming MMI or carbimazole 3-7 days after

613 RAI administration should be considered, and generally tapered as thyroid function

614 normalizes. In one study, if MMI was restarted 7 days after RAI, the free $\mathrm{T}_{4}$ measured 3

615 weeks after RAI was $6 \%$ lower than the values at the time of RAI administration, and if

616 MMI was not restarted after RAI, the free $\mathrm{T}_{4}$ values were $36 \%$ higher than the values at

617 the time of RAI administration (80). Over several decades, there have been reports that

618 pretreatment with lithium reduces the activity of RAI necessary for cure of Graves'

619 hyperthyroidism and may prevent the thyroid hormone increase seen upon ATD

620 withdrawal (81-83). However, this is not used widely, and there is insufficient evidence

621 to recommend the practice. In selected patients with Graves' hyperthyroidism who would

622 have been candidates for pretreatment with ATDs due to comorbidities or excessive

623 symptoms, but who are allergic to ATDs, the duration of hyperthyroidism may be

624 shortened by administering iodine (e.g. SSKI) beginning one week after RAI

625 administration (84).

626 [D2] Administration of RAI in the treatment of GD 
628 Sufficient activity of RAI should be administered in a single application, typically $\mathbf{a}$

629 mean dose of $10-15 \mathrm{mCi}(370-555 \mathrm{MBq})$, to render the patient with GD hypothyroid.

630 Strong recommendation, moderate-quality evidence.

631 - RECOMMENDATION 9

632 A pregnancy test should be obtained within 48 hours prior to treatment in any woman

633 with childbearing potential who is to be treated with RAI. The treating physician

634 should obtain this test and verify a negative result prior to administering RAI. Strong

635 recommendation, low-quality evidence

The goal of RAI therapy in GD is to control hyperthyroidism by rendering the

637 patient hypothyroid; this treatment is very effective, provided sufficient radiation dose is

638 deposited in the thyroid. This can be accomplished equally well by either administering a

639 fixed activity or by calculating the activity based on the size of the thyroid and its ability

640 to trap RAI (85).

641 The first method is simple, while the second method requires two unknowns to be

642 determined: the uptake of RAI and the size of the thyroid. The therapeutic RAI activity

643 can then be calculated using these two factors and the quantity of radiation $(\mu \mathrm{Ci}$ or $\mathrm{Bq})$ to

644 be deposited per gram (or cc) of thyroid (e.g., activity $(\mu \mathrm{Ci})=$ gland weight $(\mathrm{g}) \times 150-200$

$645 \mu \mathrm{Ci} / \mathrm{g} \times[1 / 24$ hour uptake in $\%$ of administered activity]). The activity in $\mu \mathrm{Ci}$ or $\mathrm{Bq}$ is

646 converted to $\mathrm{mCi}$ or $\mathrm{MBq}$ by dividing the result by 1000 . The most frequently used

647 uptake is calculated at 24 hours, and the size of the thyroid is determined by palpation or

648 ultrasound. One study found that this estimate by experienced physicians is accurate 
649 compared with anatomic imaging (86); however, other investigators have not confirmed 650 this observation (87).

651 Alternately, a more detailed calculation can be made to deposit a specific 652 radiation dose (in rad or Gy) to the thyroid. Using this approach, it is also necessary to 653 know the effective half-life of RAI (88). This requires additional time and computation 654 and, because the outcome has not shown to be better, this method is seldom used in the 655 United States. Evidence shows that to achieve a hypothyroid state, $>150 \mu \mathrm{Ci} / \mathrm{g}(5.55$ $656 \mathrm{MBq} / \mathrm{g}$ ) needs to be delivered (88-90). Patients who are on dialysis or who have 657 jejunostomy or gastric feeding tubes require special care and management when being 658 administered RAI treatment (91).

The success of RAI therapy in GD strongly depends on the administered 660 activities. In patients without adjunctive ATD, randomized controlled trials found $61 \%$ 661 success with $5.4 \mathrm{mCi}(200 \mathrm{MBq})(92), 69 \%$ with $8.2 \mathrm{mCi}(302 \mathrm{MBq})(93), 74 \%$ with 10 $662 \mathrm{mCi}(370 \mathrm{MBq})(94), 81 \%$ with $15 \mathrm{mCi}$ (555 MBq) (94) and 86\% with $15.7 \mathrm{mCi}$ (580 $663 \mathrm{MBq}$ ) (95) RAI. Due to the high rate of patients requiring retreatment, RAI therapy with 664 low activities is generally not recommended. A long-term increase in cardiovascular and cerebrovascular deaths has been

666 reported after RAI therapy not resulting in hypothyroidism as opposed to unchanged

667 mortality in RAI-treated patients on levothyroxine therapy, reflecting the role of 668 persistent hyperthyroidism as opposed to that of RAI therapy on mortality $(96,97)$. A 669 recent meta-analysis found no increase in the overall cancer risk after RAI treatment for 670 hyperthyroidism; however, a trend towards increased risk of thyroid, stomach and kidney 
671 cancer was seen, requiring further research (98). In some men, there is a modest fall in

672 the testosterone to luteinizing hormone ( $\mathrm{LH})$ ratio after RAI therapy that is subclinical

673 and reversible (99). Conception should be delayed in women until stable euthyroidism

674 is established (on thyroid hormone replacement following successful thyroid ablation).

675 This typically takes 4-6 months or longer. Conception should be delayed 3-4 months

676 in men to allow for turnover of sperm production. However, once the patient (both

677 genders) is euthyroid, there is no evidence of reduced fertility and offspring of treated

678 patients show no congenital anomalies compared to the population at large (100).

Technical remarks: Rendering the patient hypothyroid can be accomplished

680 equally well by administering either a sufficient fixed activity or calculating an activity

681 based on the size of the thyroid and its ability to trap iodine. Fetuses exposed to RAI after

682 the $10^{\text {th }}$ to $11^{\text {th }}$ week of gestation may be born athyreotic $(101,102)$ and are also at a

683 theoretical increased risk for reduced intelligence and/or cancer. In breast-feeding

684 women, RAI therapy should not be administered for at least 6 weeks after lactation stops

685 to ensure that RAI will no longer be actively concentrated in the breast tissues. A delay of

6863 months will more reliably ensure that lactation-associated increase in breast sodium

687 iodide symporter activity has returned to normal (103). Breast feeding should not be

688 resumed after RAI therapy.

- RECOMMENDATION 10

690 The physician administering RAI should provide written advice concerning radiation

691 safety precautions following treatment. If the precautions cannot be followed, 
692 alternative therapy should be selected. Strong recommendation, low-quality

693 evidence.

694 All national and regional radiation protection rules regarding RAI treatment

695 should be followed $(104,105)$. In the United States, the treating physician must ensure

696 and document that no adult member of the public is exposed to $0.5 \mathrm{mSv}$ (500 milli-

697 roentgen equivalent in man [mrem]) when the patient is discharged with a retained

698 activity of $33 \mathrm{mCi}(1.22 \mathrm{GBq})$ or greater, or emits $\geq 7 \mathrm{mrem} / \mathrm{h}(70 \mu \mathrm{Sv} / \mathrm{h})$ at $1 \mathrm{~m}$.

699 Technical remarks: Continuity of follow-up should be provided and can be

700 facilitated by communication between the referring physician and the treating physician,

701 including a request for therapy from the former and a statement from the latter that the

702 treatment has been administered.

703 [D3] Patient follow-up after RAI therapy for GD

704 - RECOMMENDATION 11

705 Follow-up within the first 1-2 months after RAI therapy for GD should include an

706 assessment of free $\mathrm{T}_{4}$, total $\mathrm{T}_{3}$, and $\mathrm{TSH}$. Biochemical monitoring should be continued

707 at 4-6 week intervals for 6 months, or until the patient becomes hypothyroid and is

708 stable on thyroid hormone replacement. Strong recommendation, low-quality

709 evidence.

Most patients respond to RAI therapy with a normalization of thyroid function

711 tests and improvement of clinical symptoms within 4-8 weeks. Hypothyroidism may 
712 occur from 4 weeks on, with $40 \%$ of patients being hypothyroid by 8 weeks and $>80 \%$ by

71316 weeks (106). This transition can occur rapidly but more commonly between 2 and 6

714 months, and the timing of thyroid hormone replacement therapy should be determined by

715 results of thyroid function tests, clinical symptoms, and physical examination. Transient

716 hypothyroidism following RAI therapy can rarely occur, with subsequent complete

717 recovery of thyroid function or recurrent hyperthyroidism (107). In such patients the

718 thyroid gland often remains palpable.

719 Beta-blockers that were instituted prior to RAI treatment should be tapered when

720 free $T_{4}$ and total $T_{3}$ have returned to the reference range. As free $T_{4}$ and total $T_{3}$ improve,

721 MMI can usually be tapered, which allows an assessment of the response to RAI.

722 Most patients eventually develop hypothyroidism following RAI, which is

723 indicated by a free $\mathrm{T}_{4}$ below normal range. At this point, levothyroxine should be

724 instituted. TSH levels may not rise immediately with the development of hypothyroidism,

725 and should not be used initially to determine the need for levothyroxine. When thyroid

726 hormone replacement is initiated, the dose should be adjusted based on an assessment of

727 free $\mathrm{T}_{4}$. The required dose may be less than the typical full replacement, and careful

728 titration is necessary owing to nonsuppressible residual thyroid function. Overt

729 hypothyroidism should be avoided, especially in patients with active GO (see section

730 U2). Once euthyroidism is achieved, lifelong annual thyroid function testing is

731 recommended at least annually, or if the patietn experiences symptoms of

732 hypothyroidism or hyperthyroidism. 
734 longer after hyperthyroidism resolves, the levels should be interpreted cautiously and 735 only in concert with free $\mathrm{T}_{4}$ and total $\mathrm{T}_{3}$.

736 [D4] Treatment of persistent Graves' hyperthyroidism following RAI therapy

737 - RECOMMENDATION 12

738 When hyperthyroidism due to GD persists after 6 months following RAI therapy,

739 retreatment with RAI is suggested. In selected patients with minimal response 3

740 months after therapy additional RAI may be considered. Weak recommendation,

$741 \quad$ low-quality evidence.

Technical remarks: Response to RAI therapy can be assessed by monitoring the

743 size of the gland, thyroid function, and clinical signs and symptoms. The goal of

744 retreatment is to control hyperthyroidism with certainty by rendering the patient

745 hypothyroid. Patients who have persistent, suppressed TSH with normal total $\mathrm{T}_{3}$ and free

$746 \mathrm{~T}_{4}$ may not require immediate retreatment but should be monitored closely for either

747 relapse or development of hypothyroidism. In the small percentage of patients with

748 hyperthyroidism refractory to several applications of RAI, surgery should be considered 749 (108). 
ATDs have been employed for seven decades (109). The goal of the therapy is to

754 render the patient euthyroid as quickly and safely as possible. These medications do not

755 cure Graves' hyperthyroidism. However, when given in adequate doses, they are very

756 effective in controlling the hyperthyroidism; when they fail to achieve euthyroidism, the

757 usual cause is nonadherence (110). The treatment itself might have a beneficial

758 immunosuppressive role, either to primarily decrease thyroid specific autoimmunity, or

759 secondarily, by ameliorating the hyperthyroid state, which may restore the dysregulated

760 immune system back to normal (111). In fact, the rate of remission with ATD therapy is

761 much higher (112) than the historical rates of spontaneous remission (113).

762 [E1] Initiation of antithyroid drug therapy for the treatment of GD

- RECOMMENDATION 13

764 Methimazole should be used in virtually every patient who chooses ATD therapy for

765 GD, except during the first trimester of pregnancy when propylthiouracil (PTU) is

766 preferred, in the treatment of thyroid storm, and in patients with minor reactions to

767 MMI who refuse radioactive iodine therapy or surgery. Strong recommendation,

768 moderate-quality evidence.

769 - RECOMMENDATION 14

770 Patients should be informed of side effects of ATDs and the necessity of informing the 771 physician promptly if they should develop pruritic rash, jaundice, acolic stools or dark 772 urine, arthralgias, abdominal pain, nausea, fatigue, fever, or pharyngitis. Preferably, 773 this should be in writing. Before starting ATDs and at each subsequent visit, the 
patient should be alerted to stop the medication immediately and call their physician

Prior to initiating ATD therapy for GD, we suggest that patients have a baseline complete blood count, including white count with differential, and a liver profile including bilirubin and transaminases. Weak recomendation, low-quality evidence.

In the United States, MMI and PTU are available, and in some countries,

782 carbimazole, a precursor of MMI, is widely used. Carbimazole is rapidly converted to

783 MMI in the serum (10 $\mathrm{mg}$ of carbimazole is metabolized to approximately $6 \mathrm{mg}$ of

784 MMI). They work in an identical fashion and both will be referred to as MMI in this text.

785 Both are effective as a single daily dose. At the start of MMI therapy, initial doses of 10-

$78630 \mathrm{mg}$ daily are used to to restore euthyroidism, and then the dose can be titrated down to

787 a maintenance level (generally 5-10 mg daily) (109,114). The dose of MMI should be

788 targeted to the degree of thyroid dysfunction, as too low a dose will not restore a

789 euthyroid state in patients with severe disease (115), and an excessive dose can cause

790 iatrogenic hypothyroidism in patients with mild disease (116). In addition, adverse drug

791 reactions are more frequent with higher MMI doses. Thus, it is important to use an MMI

792 dose that will achieve the clinical goal of normalization of thyroid function reasonably

793 rapidly, while minimizing adverse drug effects. The task force suggests the following

794 as a rough guide to initial MMI daily dosing: 5-10 $\mathrm{mg}$ if free $\mathrm{T}_{4}$ is $1-1.5$ times the upper

795 limit of normal (ULN); 10-20 mg for free $\mathrm{T}_{4}$ 1.5-2 times the ULN; 30-40 mg for free $\mathrm{T}_{4}$ 
2-3 times the ULN. These rough guidelines should be tailored to the individual patient,

797 incorporating additional information on symptoms, gland size and total $\mathrm{T}_{3}$ levels where

798 relevant. Serum $T_{3}$ levels are important to monitor initially, as some patients normalize

799 their free $\mathrm{T}_{4}$ levels with MMI but have persistently elevated serum $\mathrm{T}_{3}$, indicating

800 continuing thyrotoxicosis (117).

801

MMI has the benefit of once-a-day administration and a reduced risk of major

803 side effects compared to PTU. PTU has a shorter duration of action and is usually

804 administered two or three times daily, starting with 50-150 mg three times daily,

805 depending on the severity of the hyperthyroidism. As the clinical findings and thyroid

806 function tests return to normal, reduction to a maintenance PTU dose of $50 \mathrm{mg}$ two or

807 three times daily is usually possible. When more rapid biochemical control is needed in

808 patients with severe thyrotoxicosis, an initial split dose of MMI (e.g., 15 or $20 \mathrm{mg}$ twice a

809 day) may be more effective than a single daily dose, as the duration of action of MMI

810 may be less than 24 hours (118). Higher doses of antithyroid medication are sometimes

811 administered continuously and combined with L-thyroxine in doses to maintain euthyroid

812 levels (so-called block and replace therapy). However, this approach is not generally

813 recommended, as it has been shown to result in a higher rate of ATD side effects

$814(109,119)$.

815 The use of potassium iodine (KI) as a beneficial adjunct to ATD therapy for

816 Graves' disease has been investigated in previous studies (120). Indeed, a recent RCT

817 described the administration of $38 \mathrm{mg}$ of potassium iodide (KI) together with $15 \mathrm{mg}$ of 
818 MMI daily, which resulted in better control of hyperthyroidism and fewer adverse

819 reactions compared to $30 \mathrm{mg}$ of MMI given alone (121).

820 [E2] Adverse effects of Antithyroid Drugs

821 In general, adverse effects of ATDs can be divided into common, minor allergic

822 side effects and rare but serious allergic/toxic events such as agranulocytosis, vasculitis,

823 or hepatic damage. In a recent systematic review of eight studies that included 667 GD

824 patients receiving MMI or PTU, 13\% of patients experienced adverse events (122). The

825 minor allergic reactions included pruritus or a limited, minor rash, in $6 \%$ of patients

826 taking MMI and 3\% of patients taking PTU (122). Hepatocellular injury occurred in

$8272.7 \%$ of patients taking PTU and $0.4 \%$ of patients taking MMI. In a separate study of

828449 GD patients receiving MMI or PTU, 24\% developed a cutaneous reaction, 3.8\%

829 developed transaminase elevations more than 3-fold above normal, and $0.7 \%$ developed

830 agranulocytosis (absolute neutrophil count (ANC) < 500) (123). Cutaneous reactions

831 were more common with PTU or higher dose MMI (30 mg/day), compared to lower dose

832 MMI (15 mg per day). Hepatotoxicity was more common with PTU. Cutaneous

833 reactions appeared after a median of 18-22 days of treatment, significantly earlier than

834 transaminase elevations (median 28 days). The percentage of patients discontinuing

835 ATD therapy was $17 \%$ in the low dose MMI group, $29 \%$ in the high dose MMI group,

836 and $34 \%$ in the PTU group (123).

837 [E3] Agranulocytosis 
Although ATD associated agranulocytosis is uncommon, it is life-threatening.

839 PTU at any dose appears to be more likely to cause agranulocytosis, compared to low

840 doses of MMI (124-126). Three recent reports of large numbers of ATD-treated patients

841 who developed hematologic complications provide information on risk factors, treatment,

842 and outcomes (127-129). Two studies were from Japan and one was from Denmark. In

843 both countries the majority of patients are treated with MMI, so data are more limited for

844 PTU-associated agranulocytosis. In the first study, a retrospective cohort analysis of over

845 50,000 GD patients, 55 developed agranulocytosis, of whom 5 had pancytopenia, for an

846 estimated cumulative incidence of $0.3 \%$ in 100 days (127), with a median interval to

847 onset of 69 days. All 50 patients with agranulocytosis alone were successfully treated

848 with granulocyte colony stimulating factor (G-CSF), steroids, or supportive care, but one

849 of five patients with pancytopenia died. No predictive risk factors for the development of

850 agranulocytosis could be identified. The second study was based on a national database

851 for adverse drug reactions, which may have included some patients reported in the first

852 study (128). Seven-hundred-fifty-four GD patients who developed ATD-induced

853 hematologic complications were reported, for an estimated incidence of $0.1-0.15 \%$. Of

854 them, 725 patients received MMI, 28 received PTU, and one received both drugs.

855 Eighty-nine percent developed agranulocytosis and $11 \%$ developed pancytopenia or

856 aplastic anemia. At the onset of agranulocytosis, the average MMI dose was $25 \mathrm{mg}$ /day

857 and the average PTU dose was $217 \mathrm{mg} / \mathrm{day}$. The average age of patients developing

858 agranulocytosis was slightly older (45 vs 40 years), an observation that has been made by

859 others. Seventy-two percent developed agranulocytosis within 60 days of starting ATD,

860 and $85 \%$ within 90 days. In $7 \%$ of patients, agranulocytosis occured later than 4 months 
861 after starting ATD, but some of these patients had discontinued the medication for long

862 periods of time and developed agranulocytosis after a second or subsequent exposure.

863 Thirty of the events (4\%) were fatal. In the third study from Denmark, the frequency of

864 agranulocytosis was $0.27 \%$ with PTU and $0.11 \%$ with MMI (129). As in prior studies,

865 the median duration of therapy prior to the development of agranulocytosis was 36 and

86638 days for MMI and PTU, respectively.

867 [E4] Hepatotoxicity

868 Hepatotoxicity is another major adverse effect of ATD therapy. MMI

869 hepatotoxicity has been described as typically cholestatic, but hepatocellular disease may

870 be seen $(130,131)$. In contrast, PTU can cause fulminant hepatic necrosis that may be

871 fatal; liver transplantation has been necessary in some patients taking PTU (132). It is for

872 this reason that in $\mathbf{2 0 1 0}$ the FDA issued a safety alert regarding the use of PTU, and an

873 analysis of FDA Medwatch data (133) concluded that children are more susceptible to

874 hepatotoxic reactions from PTU than are adults.

A recent pharmacoepidemiologic study from Taiwan challenges the concept that

876 MMI hepatotoxicity is usually cholestatic, while PTU hepatotoxicity is most often

877 hepatocellular (134). Among 71,379 new users of ATDs with a median follow-up of

878196 days, MMI was associated with a higher rate of a diagnosis of non-infectious

879 hepatitis than PTU (0.25\% versus $0.08 \%$, respectively), whereas cholestasis was not

880 different $(\mathbf{0 . 0 1 9 \%}$ versus $\mathbf{0 . 0 1 6 \%})$. A diagnosis of liver failure was more common

881 after PTU (0.048\% versus $0.026 \%$ in MMI treated patients). Similar findings were

882 also recently reported from China (135). These surprising results from Asia, which are in 
883 contrast to other data from the U.S. $(133,136)$, suggest that prior data on MMI-related

884 hepatotoxicity from small case series may need to be reconsidered. In the study from

885 Denmark (129), hepatotoxic reactions were not classified as cholestatic or hepatocellular,

886 but the frequency of "liver failure" was similar for MMI $(0.03 \%)$ and PTU $(0.03 \%)$.

[E5] Vasculitis

Aside from hematologic and hepatic adverse effects, there are other rare side

890 effects with ATDs. PTU, and rarely MMI can cause causes antineutrophil cytoplasmic

891 antibody (pANCA)-positive small vessel vasculitis $(137,138)$ as well as drug induced

892 lupus (139). The risk appears to increase with duration of therapy as opposed to other

893 adverse effects seen with ATDs that typically occur early in the course of treatment

894 (140,141). Typically, granulocyte myeloperoxidase is the targeted antigen of the ANCA,

895 but antibodies to many other proteins are seen as well (142). ANCA positive vasculitis is

896 more common in patients of Asian ethnicity, and the majority of reports come from that

897 part of the world (143). While up to $40 \%$ of patients taking PTU develop ANCA

898 positivity, the vast majority of such individuals do not develop clinical vascutitis (144).

899 When the drug is discontinued, the ANCA slowly disappear in most individuals (144).

900 Children seem to be more likely to develop PTU-related ANCA positive vasculitis (133).

901 In most cases, the vasculitis resolves with drug discontinuation, although

902 immunosuppressive therapy may be necessary (145). 
Rare cases of insulin autoimmune syndrome with symptomatic hypoglycemia

904 have been reported in patients treated with MMI $(146,147)$.

906 laboratory values should be considered before initiating ATD therapy. This is suggested

907 in part because low white cell counts are common in patients with GD and in African

908 Americans (10\% of whom have a neutrophil count under 2000 (148)), and abnormal liver

909 enzymes are frequently seen in patients with thyrotoxicosis (149). While there is no

910 evidence that neutropenia or liver disease increases the risk of complications from

911 ATDs, the opinion of the task force is that a baseline absolute neutrophil count

$912<1000 / \mathrm{mm}^{3}$ or liver transaminase enzyme levels elevated more than fivefold above the

913 upper limit of normal should prompt serious reconsideration of initiating ATD therapy. It

914 is advisable to provide information concerning side effects of ATDs to the patient both

915 verbally and in writing to assure their comprehension, and document that this has been

916 done. This information can be found online $(150,151)$.

917 [E6] Monitoring of patients taking antithyroid drugs

918 There is a need for periodic clinical and biochemical evaluation of thyroid status

919 in patients taking ATDs, and it is essential that the patient understand its importance. An

920 assessment of serum free $T_{4}$ and total $T_{3}$ should be obtained about 2 to 6 weeks after

921 initiation of therapy, depending on the severity of the thyrotoxicosis, and the dose of

922 medication adjusted accordingly. Serum $\mathrm{T}_{3}$ should be monitored because the serum free

$923 \mathrm{~T}_{4}$ levels may normalize despite persistent elevation of serum total $\mathrm{T}_{3}$. Serum TSH may 
924 remain suppressed for several months after starting therapy and is therefore not a good

925 parameter for monitoring therapy early in the course.

926 Once the patient is euthyroid, the dose of MMI can usually be decreased by 30-

$92750 \%$, and biochemical testing repeated in 4-6 weeks. Once euthyroid levels are achieved

928 with the minimal dose of medication, clinical and laboratory evaluation can be

929 undertaken at intervals of 2-3 months. If a patient is receiving long-term MMI (> 18

930 months), this interval can be increased to 6 months (see below).

931 - RECOMMENDATION 16

932 A differential white blood cell count should be obtained during febrile illness and at

933 the onset of pharyngitis in all patients taking antithyroid medication. Strong

934 recommendation, low-quality evidence.

- RECOMMENDATION 17

936 There is insufficient evidence to recommend for or against routine monitoring of white

937 blood cell counts in patients taking ATD's. No recommendation, insufficient

938 evidence to assess benefits and risks.

There is no consensus concerning the utility of periodic monitoring of white blood

940 cell counts and liver function tests in predicting early onset of adverse reaction to the

941 medication (152). While routine monitoring of white blood cell counts may detect early

942 agranulocytosis, this practice is not likely to identify cases, as the frequency is quite low

$943(0.2 \%-0.5 \%)$ and the condition is usually sudden in onset. In a recent analysis of 211 
944 patients with ATD-induced agranulocytosis who had at least one prior granulocyte count

945 measured, $21 \%$ had a normal white blood count within a week, and 53\% within two

946 weeks, before developing agranulocytosis (128). However, other patients did display a

947 gradual decline in white blood cell count prior to developing agranulocytosis, suggesting

948 that monitoring might have been useful in some affected patients (152). Because patients

949 are typically symptomatic, measuring white blood cell counts during febrile illnesses and

950 at the onset of pharyngitis has been the standard approach to monitoring. If monitoring is

951 employed, the maximum benefit would be for the first 90 days of therapy, when the vast

952 majority of agranulocytosis occurs. In a patient developing agranulocytosis or other

953 serious side effects while taking either MMI or PTU, use of the other medication is

954 contraindicated owing to risk of cross-reactivity between the two medications (153). The

955 contraindication to use PTU might be reconsidered in life-threatening thyrotoxicosis (i.e.,

956 thyroid storm) in a MMI-treated patient who has developed agranulocytosis, especially if

957 the duration of therapy is brief (154).

- RECOMMENDATION 18

959 Liver function and hepatocellular integrity should be assessed in patients taking MMI

960 or PTU who experience pruritic rash, jaundice, light-colored stool or dark urine, joint

961 pain, abdominal pain or bloating, anorexia, nausea, or fatigue. Strong

962 recommendation, low-quality evidence.

963 Hyperthyroidism can itself cause mildly abnormal liver function tests in up to

$96430 \%$ of patients (149). PTU itself may cause transient elevations of serum transaminases

965 in up to one-third of patients. Significant elevations to threefold above the upper limit of 
normal are seen in up to $4 \%$ of patients taking PTU (155), a prevalence higher than with

967 MMI. As noted above, PTU can also cause fatal hepatic necrosis, leading to the

968 suggestion by some that patients taking this ATD have routine monitoring of their liver

969 function, especially during the first 6 months of therapy. A 2009 review of the literature

970 (136) found that PTU hepatotoxicity occurred after a median of 120 days after initiation

971 of therapy. It is difficult to distinguish these abnormalities from the effect of persistent

972 thyrotoxicosis unless they are followed prospectively. In patients with improving

973 thyrotoxicosis, a rising alkaline phosphatase with normalization of other liver function

974 does not indicate worsening hepatic toxicity (156), as the origin of the alkaline

975 phosphatase is from bone, not liver. The onset of PTU-induced hepatotoxicity may be

976 acute, difficult to appreciate clinically, and rapidly progressive. If not recognized, it can

977 lead to liver failure and death (115,157-159). Routine monitoring of liver function in all

978 patients taking ATDs has not been found to prevent severe hepatotoxicity. If monitoring

979 is employed, the maximum benefit would be for the first 120 days of therapy, when the

980 vast majority of instances of hepatotoxicity occur.

$981 \quad$ Technical remarks: PTU should be discontinued if transaminase levels (found

982 incidentally or measured as clinically indicated) reach $>3$ times the upper limit of normal

983 or if levels elevated at the onset of therapy increase further. After discontinuing the

984 drug, liver function tests should be monitored weekly until there is evidence of

985 resolution. If resolution is not evident, prompt referral to a gastroenterologist or

986 hepatologist for specialty care is warranted. Except in cases of severe PTU-induced

987 hepatotoxicity, MMI can be used to control the thyrotoxicosis without ill effect

$988(160,161)$. 
990 There is insufficient information to recommend for or against routine monitoring of

991 liver function tests in patients taking ATD's. No recommendation, insufficient

992 evidence to assess benefits and risks.

993 [E7] Management of allergic reactions

994

1003 safe in the case of minor side effects, although patients may develop similar side effects

1004 with the second ATD (123). In this study, 71 patients with an adverse event from either

1005 MMI or PTU switched to the other ATD, with doses individually determined. Median

1006 dose of the second ATD was $15 \mathrm{mg} / \mathrm{d}$ for MMI (range $10-30$ ) and $300 \mathrm{mg} /$ day for PTU

1007 (range 150 - 450). Thirty-four percent of patients switched to PTU and 30\% of patients

1008 switched to MMI developed side effects, generally the same type as occurred on the 
1009 original ATD, while the remaining patients tolerated the second ATD without

1010 complications (123). There is also one recent case report of a more severe reaction to

1011 MMI consisting of rash, pruritis, tongue and throat swelling that was successfully

1012 managed with antihistamine therapy, but this is not generally recommended due to the

1013 risk of analphylaxis (162).

1014 [E8] Duration of antithyroid drug therapy for GD

1015 - RECOMMENDATION 21

1016 Measurement of TRAb levels prior to stopping ATD therapy is suggested, as it aids in

1017 predicting which patients can be weaned from the medication, with normal levels

1018 indicating greater chance for remission. Strong recommendation, moderate-quality

1019 evidence.

1020 - RECOMMENDATION 22

1021 If MMI is chosen as the primary therapy for GD, the medication should be continued

1022 for approximately $12-18$ months, then discontinued if the TSH and TRAb levels are

1023 normal at that time. Strong recommendation, high-quality evidence.

1024 - RECOMMENDATION 23

1025 If a patient with GD becomes hyperthyroid after completing a course of MMI,

1026 consideration should be given to treatment with RAI or thyroidectomy. Continued

1027 low-dose MMI treatment for longer than 12-18 months may be considered in patients 
1028 not in remission who prefer this approach. Weak recommendation, low-quality

1029 evidence.

1030 A patient is considered to be in remission if they have had a normal serum TSH,

1031 free $\mathrm{T}_{4}$, and total $\mathrm{T}_{3}$ for 1 year after discontinuation of ATD therapy. The remission rate

1032 varies considerably between geographical areas. In earlier studies in the United States,

1033 about $20 \%-30 \%$ of patients were reported to have a lasting remission after $12-18$ months

1034 of medication, (59), but more recent data are not available. The remission rate may be

1035 higher in Europe and Japan; a long-term European study indicated a 50-60\% remission

1036 rate after 5-6 years of treatment (163), and a study in Japan reported a 68\% remission

1037 rate after 2 years of treatment (164). A meta-analysis shows the remission rate in adults is

1038 not improved by a course of ATDs longer than 18 months (119). A lower remission rate

1039 has been described in men, smokers (especially men), and those with large goiters $(\geq 80$

1040 g) (165-169). Higher initial doses of MMI (60-80 mg/day) do not improve remission

1041 rates, but increase the risk of side effects, and are not recommended (170).

1042 TRAb assessment at the end of the course of ATD therapy is a useful method of

1043 dividing patients into 2 groups: one with persistent elevations who are unlikely to be in

1044 remission, and another group with low or undetectable TRAb, who have a higher

1045 probability of permanent remission $(171,172)$. In the group with elevated TRAb, relapse

1046 rates approach $80-100 \%$, while in the latter group, relapse rates are in the $20-30 \%$ range

$1047(171,172)$.

1048 [E9] Persistently elevated TRAb 
1049 Patients with persistently high TRAb could continue ATD therapy (and repeat

1050 TRAb after an additional 12-18 months) or opt for alternate definitive therapy with RAI

1051 or surgery. In selected patients (i.e., younger patients with mild stable disease on a low

1052 dose of MMI), long-term MMI is a reasonable alternative approach $(65,173)$. Another

1053 study by the same author reported that MMI doses of $2.5-10 \mathrm{mg} /$ day for a mean of 14

1054 years were safe and effective for the control of GD in 59 patients (174). A recent

1055 retrospective analysis compared long term outcomes (mean follow up period of 6-7

1056 years) of patients who had relapsed after a course of ATDs, who were treated with either

1057 RAI and levothyroxine or long-term ATD therapy (175). Those patients treated with RAI

$1058(\mathrm{n}=114)$ more often had persistent thyroid eye disease, continuing thyroid dysfunction,

1059 and experienced more weight gain compared to those patients receiving long-term ATD

1060 treatment $(n=124)$.

1061 If continued MMI therapy is chosen, TRAb levels might be monitored every 1-2

1062 years, with consideration of MMI discontinuation if TRAb levels become negative over

1063 long term follow up. For patients choosing long-term MMI therapy, monitoring of

1064 thyroid function every 4-6 months is reasonable, and patients can be seen for follow up

1065 visits every 6-12 months.

1066 [E10] Negative TRAb

1067 If TRAb is negative and thyroid function is normal at the end of 12-18 months

1068 of MMI therapy, it is reasonable to discontinue the drug. If a patient experiences a

1069 relapse in follow up, RAI therapy or surgery should be considered. 
1071 relatively later than those that develop in patients whose MMI is stopped when TRAb is 1072 still positive $(171,176)$, although 5\% occurred within the first 2 months (167). Therefore,

1073 in this population, thyroid function testing should be monitored at 2 to 3 -month intervals

1074 for the first 6 months, then at 4 to 6-month intervals for the next 6 months, then every 6

1075 to 12 months, in order to detect relapses as early as possible. The patient should be

1076 counseled to contact the treating physician if symptoms of hyperthyroidism are

1077 recognized. Should a relapse occur, patients should be counseled about alternatives for

1078 therapy, which would include another course of MMI, RAI, or surgery. If ATD therapy

1079 is chosen, patients should be aware that agranulocytosis can occur with a second

1080 exposure to a drug, even many years later, despite an earlier uneventful course of therapy

$1081(177,178)$. If the patient remains euthyroid for more than 1 year (i.e., they are in

1082 remission), thyroid function should be monitored at least annually, as relapses can occur

1083 years later (171), and some patients eventually become hypothyroid (179).

1085 [F] If thyroidectomy is chosen for treatment of GD, how should it be accomplished?

1086 [F1] Preparation of patients with GD for thyroidectomy

1087 - RECOMMENDATION 24

1088 If surgery is chosen as treatment for GD, patients should be rendered euthyroid prior 1089 to the procedure with ATD pretreatment, with or without beta-adrenergic blockade. A 
1090 potassium iodide containing preparation should be given in the immediate

1091 preoperative period. Strong recommendation, low-quality evidence.

1092

1093

1094

1095

1096

1097

1098

1099

1100

1101

1102

1103

1104

1105

1106

1107 MMI before undergoing surgery (180). Preoperative potassium iodide, saturated solution

1108 of potassium iodide (SSKI), or Lugol's solution should be used before surgery in most

1109 patients with GD. This treatment is beneficial as it decreases thyroid blood flow,

1110 vascularity, and intraoperative blood loss during thyroidectomy $(181,182)$. In a recent 
1111 series of 162 patients with GD and 102 patients with TMNG, none of whom received

1112 SSKI preoperatively, there were no significant differences in operative times, blood loss,

1113 or postoperative complications between the two groups; the authors concluded that

1114 omitting preoperative SSKI for GD patients does not impair patient outcomes (183).

1115 Given that this study was performed at a single high-volume institution, its findings may

1116 not be generalizable; comparison was made between two different pathologies, and there

1117 was no comparison group of patients with GD who received SSKI. It is unclear also

1118 whether it was adequately powered to detect a significant difference, if one existed.

1119 However this study mitigates concern when thyroidectomy is scheduled and SSKI is not

1120 given because of shortages, scheduling issues, patient allergy or patient intolerance. In

1121 addition, rapid preparation for emergent surgery can be facilitated by the use of

1122 corticosteroids (184), and potentially cholestyramine (185-187).

1123 Technical remarks: Potassium iodide can be given as 5-7 drops $(0.25-0.35 \mathrm{~mL})$

1124 of Lugol's solution (8 mg iodide/drop) or 1-2 drops (0.05-0.1 mL) of SSKI (50 mg

1125 iodide/drop) three times daily mixed in water or juice for 10 days before surgery.

1126 Recent data suggest that supplementing oral calcium and/or vitamin D

1127 preoperatively may reduce the risk of postoperative hypocalcemia due to parathyroid

1128 injury or increased bone turnover (188). Oltmann et al compared 45 Graves' patients

1129 treated with $1 \mathrm{~g}$ oral calcium carbonate three times a day for two weeks prior to surgery

1130 to 38 Graves' patients who underwent thyroidectomy without treatment, as well as 38

1131 euthyroid controls; rates of biochemical and symptomatic hypocalcemia were

1132 significantly higher in non-treated Graves' patients compared to the two other treatment 
1133 groups (189). Another study that focused on postoperative hypocalcemia after thyroid

1134 surgery for thyroid cancer, not hyperthyroidism, identified a reduction in postoperative

1135 symptomatic hypocalcemia when patients have preoperative serum 25-hydroxy vitamin

1136 D levels $>20 \mathrm{ng} / \mathrm{ml}(>8 \mathrm{nmol} / \mathrm{l})$ prior to the operating room (190). A meta-analysis of risk

1137 factors for postoperative hypocalcemia identified preoperative vitamin D deficiency as a

1138 risk factor for postoperative hypocalcemia, as well as GD itself (188). In two studies

1139 included in another meta-analysis, supplementing calcitriol for a brief period

1140 preoperatively helped reduce transient post-thyroidectomy hypocalcemia (191-193).

$1141[\mathrm{~F} 2]$ The surgical procedure and choice of surgeon

\section{2 - RECOMMENDATION 27}

1143 If surgery is chosen as the primary therapy for GD, near-total or total thyroidectomy is

1144 the procedure of choice. Strong recommendation, moderate-quality evidence.

1145 Thyroidectomy has a high cure rate for the hyperthyroidism of GD. Total

1146 thyroidectomy has a nearly $0 \%$ risk of recurrence, whereas subtotal thyroidectomy may

1147 have an 8\% chance of persistence or recurrence of hyperthyroidism at 5 years (194-197).

1148 The most common complications following near-total or total thyroidectomy are

1149 hypocalcemia due to hypoparathyroidism (which can be transient or permanent),

1150 recurrent or superior laryngeal nerve injury (which can be temporary or permanent),

1151 postoperative bleeding, and complications related to general anesthesia.

1152 - RECOMMENDATION 28 
1153 If surgery is chosen as the primary therapy for GD, the patient should be referred to a

1154 high-volume thyroid surgeon. Strong recommendation, moderate-quality evidence.

1155 Improved patient outcome has been shown to be independently associated with

1156 high thyroidectomy surgeon volume; specifically, average complication rates, length of

1157 hospital stay, and cost are reduced when the operation is performed by a surgeon who

1158 conducts many thyroidectomies. A significant association is seen between increasing

1159 thyroidectomy volume and improved patient outcome; the association is robust and is

1160 more pronounced with an increasing number of thyroidectomies $(198,199)$. Data show

1161 that surgeons who perform more than 25 thyroid surgeries per year have superior patient

1162 clinical and economic outcomes compared to those who perform fewer; complication

1163 rates are $51 \%$ higher on average when surgery is performed by low-volume

1164 surgeons $(62,199,200)$. The surgeon should be thoroughly trained in the procedure,

1165 have an active practice in thyroid surgery, and have conducted a significant number of

1166 thyroidectomies with a low frequency of complications. Following thyroidectomy for GD

1167 in the hands of high-volume thyroid surgeons, the rate of permanent hypoparathyroidism

1168 has been determined to be $<2 \%$, and permanent recurrent laryngeal nerve (RLN) injury

1169 occurs in $<1 \%$ (201). The frequency of bleeding necessitating reoperation is $0.3 \%-0.7 \%$

1170 (202). Mortality following thyroidectomy is between 1 in 10,000 and 5 in 1,000,000

1171 (203).

$1172 \quad[\mathrm{~F} 3]$ Postoperative care

1173 — RECOMMENDATION 29 
1174 Following thyroidectomy for GD, alternative strategies may be undertaken for

1175 management of calcium levels: serum calcium \pm intact parathyroid hormone levels can

1176 be measured, and oral calcium and calcitriol supplementation administered based on

1177 these results, or prophylactic calcium with or without calcitriol prescribed empirically.

1178 Weak recommendation, low-quality evidence.

1179 Successful prediction of calcium status after total thyroidectomy can be achieved

1180 using the slope of 6- and 12-hour postoperative calcium levels (204-210). Postoperative

1181 routine supplementation with oral calcium and calcitriol decreases development of

1182 hypocalcemic symptoms and intravenous calcium requirement, allowing for safer early

1183 discharge (211). Low iPTH levels $(<10-15 \mathrm{pg} / \mathrm{mL})$ in the immediate postoperative

1184 setting appear to predict symptomatic hypocalcemia and need for calcium and calcitriol

1185 (1,25 vitamin D) supplementation (212,213). However, normal levels of serum iPTH may

1186 not predict eucalcemia for GD patients (214). Vitamin D insufficiency may serve as an

1187 underlying cause.

1189 Patients can be discharged if they are asymptomatic and their serum calcium

1190 levels corrected for albumin are $8.0 \mathrm{mg} / \mathrm{dL}(2.0 \mathrm{mmol} / \mathrm{L})$ or above and are not falling

1191 over a 24-hr period. The use of ionized calcium measurements are preferred by some, and

1192 are helpful if the patient has abnormal levels of serum proteins. Intravenous calcium

1193 gluconate should be readily available and may be administered if patients have worsening

1194 hypocalcemic symptoms despite oral supplementation and/or their concomitant serum

1195 calcium levels are falling despite oral repletion. In patients with severe hypocalcemia, 
1196 teriparatide administration has yielded encouraging preliminary results (elimination of

1197 symptoms and earlier hospital discharge), but more data are needed before it can be

1198 considered for clinical practice (215). Persistent hypocalcemia in the postoperative period

1199 should prompt measurement of serum magnesium and possible magnesium repletion

1200 (216,217). In addition to reduced serum calcium levels, reduced serum phosphate and

1201 increased serum potassium levels may be observed in hungry bone syndrome. Following

1202 discharge, serum iPTH levels should be measured in the setting of persistent

1203 hypocalcemia to determine if permanent hypoparathyroidism is truly present or whether

1204 "bone hunger" is ongoing. As the patient reaches eucalcemia, calcium and calcitriol

1205 therapy can be tapered.

1206 Technical remarks: Calcium supplementation can be accomplished with oral

1207 calcium (usually calcium carbonate, $1250-2500 \mathrm{mg}$, equivalent to 500-1000 mg

1208 elemental calcium) four times daily, tapered by $500 \mathrm{mg}$ of elemental calcium every 2

1209 days, or $1000 \mathrm{mg}$ every 4 days as tolerated. In addition, calcitriol may be started at a dose

1210 of $0.5 \mathrm{mcg}$ daily and continued for 1-2 weeks (218) and increased or tapered according to

1211 the calcium and/or iPTH level. Patients can be discharged if they are asymptomatic and

1212 have stable serum calcium levels. Postoperative evaluation is generally conducted 1-2

1213 weeks following discharge with continuation of supplementation based on clinical

1214 parameters.

1215 - RECOMMENDATION 30 
1216 ATD should be stopped at the time of thyroidectomy for GD, and beta-adrenergic

1217 blockers should be weaned following surgery. Strong recommendation, low-quality

$1218 \quad$ evidence.

1220 Following thyroidectomy for GD, L-thyroxine should be started at a daily dose

1221 appropriate for the patient's weight $(0.8 \mu \mathrm{g} / \mathrm{lb}$ or $1.6 \mu \mathrm{g} / \mathrm{kg})$, with elderly patients

1222 needing somewhat less, and serum TSH measured 6-8 weeks postoperatively. Strong

1223 recommendation, low-quality evidence.

Technical remarks: If TSH was suppressed preoperatively, a free $\mathbf{T}_{\mathbf{4}}$ and TSH

1225 should be measured 6-8 weeks postoperatively, since recovery of the pituitary-

1226 thyroid axis is occasionally delayed. The appropriate dosing of L-thyroxine will vary

1227 with patient BMI (219), and the percent of levothyroxine absorbed from the gut. Once

1228 stable and normal, TSH should be measured annually or more frequently if clinically

1229 indicated.

1230 - RECOMMENDATION 32

1231 Communication among different members of the multidisciplinary team is essential,

1232 particularly during transitions of care in the pre- and postoperative settings. Strong

1233 recommendation, low-quality evidence.

1234 It is important to assure that adequate communication occurs between the medical

1235 team and the treating surgeon to assure that euthyroidism is achievable prior to surgical 
1236 intervention; in addition, if the patient is noted to have significant vitamin D deficiency,

1237 preoperative vitamin D repletion could be performed and surgery scheduled to permit

1238 this. Important intraoperative findings and details of postoperative care, including

1239 calcium supplementation needs and management of surgical hypothyroidism, should be

1240 communicated by the surgeon to the patient and the other physicians who will be

1241 important in the patient's postoperative care (220).

1242

1243 [G] How should thyroid nodules be managed in patients with GD?

1244 — RECOMMENDATION 33

1245 If a thyroid nodule is discovered in a patient with GD, the nodule should be evaluated

1246 and managed according to recently published guidelines regarding thyroid nodules in

1247 euthyroid individuals. Strong recommendation, moderate-quality evidence.

1248 Thyroid cancer occurs in GD with a frequency of $2 \%$ or less (221). Thyroid

1249 nodules larger than $1-1.5 \mathrm{~cm}$ should be evaluated before RAI therapy. If a radioactive

1250 iodine scan is performed, any nonfunctioning or hypo-functioning nodules should be

1251 considered for fine needle aspiration (FNA), as these may have a higher probability of

1252 being malignant (62). If the cytopathology is suspicious or diagnostic of malignancy,

1253 surgery is advised after normalization of thyroid function with ATDs. Surgery should

1254 also be considered for indeterminate cytology. Disease-free survival at 20 years is

1255 reported to be $99 \%$ after thyroidectomy for GD in patients with small $(\leq 1 \mathrm{~cm})$ coexisting

1256 thyroid cancers (222). 
1258 identify more nodules and cancer than does palpation and ${ }^{123} \mathrm{I}$ scintigraphy. However,

1259 since most of these cancers are papillary microcarcinomas with minimal clinical impact,

1260 further study is required before routine ultrasound (which may lead to surgery) can be

1261 recommended $(223,224)$.

1263 for patients with thyroid nodules and differentiated thyroid cancer (225).

\section{- RECOMMENDATION 34}

1267 The diagnosis of thyroid storm should be made clinically in a severely thyrotoxic

1268 patient with evidence of systemic decompensation. Adjunctive use of a sensitive

1269 diagnostic system should be considered. Patients with a Burch-Wartofsky Point Scale

1270 (BWPS) of $\geq 45$ or Japanese Thyroid Association (JTA) categories of TS1 or TS2

1271 with evidence of systemic decompensation require aggressive therapy. The decision

1272 to use aggressive therapy in patients with a BWPS of 25-44 should be based on

1273 clinical judgment. Strong recommendation, moderate-quality evidence.

\section{4 - RECOMMENDATION 35}

1275 A multimodality treatment approach to patients with thyroid storm should be used,

1276 including beta-adrenergic blockade, antithyroid drug therapy, inorganic iodide, 
corticosteroid therapy, cooling with acetaminophen and cooling blankets, volume

1278 resuscitation, nutritional support, respiratory care and monitoring in an intensive care

1279 unit, as appropriate for an individual patient. Strong recommendation, low-quality

$1280 \quad$ evidence.

1281 Life-threatening thyrotoxicosis or thyroid storm is a rare disorder characterized by

1282 multisystem involvement and mortality rates in the range of $8-25 \%$ in modern series

$1283(25,72,226,227)$. A high index of suspicion for thyroid storm should be maintained in

1284 patients with thyrotoxicosis associated with any evidence of systemic decompensation.

1285 Diagnostic criteria for thyroid storm in patients with severe thyrotoxicosis were first

1286 proposed in 1993 and subsequently widely adopted as the Burch-Wartofsky Point Scale

1287 for thyroid storm $(26,72,186,226,228)$. These criteria (Table 6) include hyperpyrexia,

1288 tachycardia, arrhythmias, congestive heart failure, agitation, delirium, psychosis, stupor

1289 and coma, as well as nausea, vomiting, diarrhea, hepatic failure, and the presence of an

1290 identified precipitant (26). Points are awarded in the BWPS system based on the severity

1291 of individual manifestations, with a point total of $\geq 45$ consistent with thyroid storm, 25-

129244 points classified as impending thyroid storm, and $<25$ points making thyroid storm

1293 unlikely. Recently, an additional empirically defined diagnostic system has been

1294 proposed by the Japanese Thyroid Association (JTA) (72). The JTA system uses

1295 combinations of similar clinical features to assign patients to the diagnostic categories

1296 thyroid storm 1 (TS1) or thyroid storm 2 (TS2).

1297 Data comparing these two diagnostic systems suggest an overall agreement, but a

1298 tendency toward under-diagnosis using the JTA categories of TS1 and TS2, compared to 
1299 a BWPS $\geq 45(72,186,226,227)$. In a recent study including 25 patients with a clinical

1300 diagnosis of thyroid storm, the BWPS was $\geq 45$ in 20 patients and $25-44$ in the remaining

1301 five, but these latter five patients (20\%) were not identified using the JTA system (226).

1302 Importantly, in the same series, among 125 patients hospitalized with a clinical

1303 diagnosis of compensated thyrotoxicosis but not in thyroid storm, 27 (21.6\%) had a

1304 BWPS $\geq 45$, and $21(16.8 \%)$ were either TS1 or TS2, suggesting similar rates of over-

1305 diagnosis with these two systems. However, an additional 50 patients (40\%) hospitalized

1306 with a clinical diagnosis of thyrotoxicosis without thyroid storm would have been

1307 diagnosed as having impending thyroid storm by the BWPS, which reinforces that a

1308 BWPS in the 25-44 range does not supplant clinical judgment in the selection of patients

1309 for aggressive therapy.

1310 In summary, the diagnosis of thyroid storm remains a clinical one that is

1311 augmented by current diagnostic systems. A BWPS $\geq 45$ appears more sensitive than a

1312 JTA classification of TS1 or TS2 in detecting patients with a clinical diagnosis of thyroid

1313 storm, but patients with a BWPS of 25-44 represent a group in whom the decision to use

1314 aggressive therapy should be based on sound clinical judgment, and not based solely on

1315 diagnostic category, in order to avoid over-treatment and the resultant risk of drug

1316 toxicity. At a minimum, patients in this intermediate category should be observed closely

1317 for deterioration. Care should be taken with either system to avoid inappropriate

1318 application to patients without severe thyrotoxicosis as each of the manifestations of

1319 thyroid storm, with the possible exception of severe hyperpyrexia, may also be seen in 
1320 the presence of any major illness, many of which are also known precipitants of thyroid 1321 storm (186).

1322 Precipitants of thyroid storm in a patient with previously compensated

1323 thyrotoxicosis include abrupt cessation of ATDs, thyroidectomy, or nonthyroidal surgery

1324 in a patient with unrecognized or inadequately treated thyrotoxicosis, and a number of

1325 acute illnesses unrelated to thyroid disease $(72,186,228)$. Thyroid storm occasionally

1326 occurs following RAI therapy.

1327 Aggressive treatment for thyroid storm involves the early targeting of each

1328 pharmacologically accessible step in thyroid hormone production and action (Table 7).

1329 Treatment strategy for thyroid storm can be broadly divided into 1) therapy directed

1330 against thyroid hormone secretion; 2) measures directed against the peripheral action of

1331 thyroid hormone at the tissue level; 3) reversal of systemic decompensation; 4) treatment

1332 of the precipitating event or intercurrent illness; and 5) definitive therapy (26). A number

1333 of therapeutic measures are specifically intended to decrease $\mathrm{T}_{4}$-to- $\mathrm{T}_{3}$ conversion, such as

1334 the preferential use of PTU over MMI $(229,230)$, glucocorticoid therapy $(231)$, and the

1335 use of beta adrenergic blocking agents such as propranolol, with selective ability to

1336 inhibit type 1 deiodinase (232). For example, an early article comparing acute changes in

1337 thyroid hormone level after initiation of PTU or MMI found that $\mathrm{T}_{3}$ levels dropped by

1338 approximately $45 \%$ in the first 24 hours of PTU therapy compared to an approximately

1339 10-15\% decrease after starting MMI (229). Both plasmapheresis/ plasma exchange and

1340 emergency surgery have been used to treat thyroid storm in patients who respond poorly

1341 to traditional therapeutic measures $(233,234)$. 
1343 precipitants, patient education to avoid abrupt discontinuation of ATD therapy, and

1344 ensuring that patients are euthyroid prior to elective surgery, labor and delivery, or other

1345 acute stressors.

1346 Technical remarks: Treatment with inorganic iodine (SSKI/Lugol's solution), or

1347 oral cholecystographic agents (235) leads to rapid decreases in both $\mathrm{T}_{4}$ and $\mathrm{T}_{3}$ levels.

1348 Combined with ATDs in patients with severe thyrotoxicosis, these agents result in rapid

1349 clinical improvement (120). Unfortunately, the oral radiographic contrast agents ipodate

1350 and iopanoic acid are not currently available in many countries.

\section{[I] Is there a role for iodine as primary therapy in the treatment of GD?}

Prior to the introduction of ATDs, iodine was commonly reported to ameliorate

1354 the hyperthyroidism associated with GD $(236,237)$. Iodine acutely lowers thyroid

1355 hormone concentrations by reducing hormone secretion $(238,239)$, and inhibits its own

1356 organification (the Wolff-Chaikoff effect) (240). However, reports of escape from these

1357 beneficial effects of iodine (241), as well as reports of iodine induced hyperthyroidism in

1358 patients with nodular goiter (242), discouraged the use of iodine in GD. Recent studies

1359 have suggested a potential role for iodine in patients who have had adverse reactions to

1360 ATD and who also have a contraindication or aversion to RAI or surgery $(243,244)$. 


\section{RECOMMENDATION 36}

1363 Potassium iodide may be of benefit in select patients with hyperthyroidism due to GD,

1364 who have adverse reactions to ATDs, and have a contraindication or aversion to RAI

1365 therapy (or aversion to repeat RAI therapy) or surgery. Treatment may be more

1366 suitable for patients with mild hyperthyroidism, or a prior history of RAI therapy. No

1367 recommendation, insufficient evidence to assess benefits or risks.

Among 44 Japanese patients who had adverse reactions to ATD and who were

1369 treated with KI alone, 66\% were well-controlled for an average of 18 years (range 9-28

1370 years), and 39\% achieved a remission after 7 years (range 2-23 years) (243). Among the

1371 responders, the doses used were between 13 and $100 \mathrm{mg}$, and were adjusted depending

1372 upon biochemical response. Among 15 nonresponders, 11 (25\% of all patients) escaped

1373 the inhibitory effects of iodine and 4 patients did not respond at all to KI. None of the

1374 patients had side effects. Initial free $\mathrm{T}_{4}$ concentration and goiter size did not predict a

1375 response to therapy. Among 20 Japanese patients with mild hyperthyroidism initially

1376 treated with KI alone, and matched using propensity score analysis with patients treated

1377 with MMI alone, $85 \%$ of the patients treated with KI alone had normal thyroid function at

13786 months and 1 year, comparable to that of the matched controls treated with MMI (244).

1379 Most patients were treated with $50 \mathrm{mg}$ KI daily.

1380 The inhibitory effects of iodine are greater in patients with a prior history of RAI

1381 exposure (245) suggesting a role for $\mathrm{KI}$ in patients who remain hyperthyroid after one

1382 dose of RAI and prefer to avoid a second dose. The use of KI prior to thyroidectomy for

1383 GD is discussed in section [F1], the use of KI as adjunctive therapy following RAI is 
1384 discussed in section [D1], the use of KI in combination with MMI for treating GD is

1385 discussed in section [E1], and the use of KI in hyperthyroidism complicating pregnancy

1386 is discussed in section [T].

1388 [J] How should overt hyperthyroidism due to TMNG or TA be managed?

1389 - RECOMMENDATION 37

1390 We suggest that patients with overtly TMNG or TA be treated with RAI therapy or

1391 thyroidectomy. On occasion, long-term, low-dose treatment with MMI may be

1392 appropriate. Weak recommendation, moderate-quality evidence.

There are two effective and relatively safe definitive treatment options for

1394 TMNG and TA, RAI therapy and thyroid surgery. The decision regarding treatment

1395 should take into consideration a number of clinical and demographic factors, as well as

1396 patient preference. The goal of therapy is the rapid and durable elimination of the

1397 hyperthyroid state.

1398 For patients with TMNG, the risk of treatment failure or need for repeat

1399 treatment is $<1 \%$ following near-total and/total thyroidectomy $(246,247)$, compared

1400 with a $20 \%$ risk of the need for retreatment following RAI therapy $(246,248)$.

1401 Euthyroidism is achieved within days after surgery $(246,247)$. On the other hand, the

1402 risk of hypothyroidism and the requirement for exogenous thyroid hormone therapy is

$1403100 \%$ after near-total/total thyroidectomy. For patients with TMNG who receive RAI 
1404 therapy, the response is $50-60 \%$ by 3 months, and $80 \%$ by 6 months $(246,248,249)$. In

1405 a large study of patients with TMNG treated with RAI, the prevalence of

1406 hypothyroidism was $3 \%$ at 1 year and $64 \%$ at 24 years (250). Hypothyroidism was

1407 more common among patients under 50 years of age, compared with those over 70

1408 years (61\% vs. $36 \%$ after 16 years). In a more recent study, the prevalence of

1409 hypothyroidism was $4 \%$ at 1 year and $16 \%$ at 5 years (251).

1410 In large retrospective series of patients with TMNG presenting with

1411 compressive symptoms, all patients undergoing total thyroidectomy had resolution of

1412 these symptoms after treatment, whereas only $46 \%$ of patients undergoing RAI had

1413 improvement in such symptoms (252). This may be due in part to the fact that very

1414 large goiters treated with high-activity radioactive iodine only decrease in size by $30 \%-$

$141550 \%(253)$

1416 For patients with TA, the risk of treatment failure is $<1 \%$ after surgical resection

1417 (ipsilateral thyroid lobectomy or isthmusectomy) (254). Typically, euthyroidism is

1418 achieved within days after surgery. The prevalence of hypothyroidism varies from 2-

$14193 \%$ following lobectomy for TA, although rates of hypothyroidism after lobectomy for

1420 non-toxic nodules have been reported to be as high as $20 \%$ (254-256), and lower after

1421 isthmusectomy in the unique circumstance where the TA is confined to the thyroid

1422 isthmus. For patients with TA who receive RAI therapy there is a $6 \%-18 \%$ risk of

1423 persistent hyperthyroidism and a 3\%- 5.5\% risk of recurrent hyperthyroidism

1424 (254,257). There is a 75\% response rate by 3 months and $89 \%$ rate by 1 year following

1425 RAI therapy for TA $(225,257,258)$. The prevalence of hypothyroidism after RAI is 
1426 progressive and hastened by the presence of antithyroid antibodies or a nonsuppressed

1427 TSH at the time of treatment $(257,259,260)$. A study following 684 patients with TA

1428 treated with RAI reported a progressive increase in overt and subclinical

1429 hypothyroidism (259). At 1 year, the investigators noted a $7.6 \%$ prevalence, with $28 \%$

1430 at 5 years, $46 \%$ at 10 years, and $60 \%$ at 20 years. They observed a faster progression to

1431 hypothyroidism among patients who were older and who had incomplete TSH

1432 suppression (correlating with only partial extranodular parenchymal suppression) due to

1433 prior therapy with ATDs. The nodule is rarely eradicated in patients with TA

1434 undergoing RAI therapy, which can lead to the need for continued surveillance

$1435(225,257,260)$.

1436 Potential complications following near-total/total thyroidectomy include the risk 1437 of permanent hypoparathyroidism $(<2.0 \%)$ or RLN injury $(<2.0 \%)(261,262)$. There is 1438 a small risk of permanent RLN injury with surgery for TA (254). Following RAI

1439 therapy, there have been reports of new-onset GD (up to 4\% prevalence) (263), as well

1440 as concern for thyroid malignancy $(254,264,265)$ and a very minimal increase in late

1441 non-thyroid malignancy (265). Overall, the success rate of RAI (definitive

1442 hypothyroidism or euthyroidism) is high: $93.7 \%$ in TA and $81.1 \%$ in TMNG patients 1443 (266).

1444 Technical remarks: Once the diagnosis has been made, the treating physician

1445 and patient should discuss each of the treatment options, including the logistics,

1446 benefits, expected speed of recovery, drawbacks, side effects, and costs. This sets the

1447 stage for the physician to make a recommendation based upon best clinical judgment 
1448 and for the final decision to incorporate the personal values and preferences of the

1449 patient. Autonomy is an uncommon cause of hyperthyroidism in pregnancy and there

1450 is a lack of studies in this setting. However, considering the theoretical risks associated

1451 with surgery or ATD therapy (has to be used throughout pregnancy and there is a

1452 tendency to overtreat the fetus), the optimal therapy might be definitive therapy with

1453 RAI or surgery in advance of a planned pregnancy. Most experts prefer to avoid the use

1454 of RAI within 6 months of a pregnancy; it should be used with caution if at all.

1455 The panel agreed that TMNG and TA with high nodular RAI uptake and widely

1456 suppressed RAI uptake in the perinodular thyroid tissue are especially suitable for RAI

1457 therapy. However, there are insufficient data to make a recommendation based on these 1458 findings.

1460 Factors that favor a particular modality as treatment for TMNG or TA (Table

$14618)$

1462 a. RAI therapy: Advanced patient age, significant comorbidity, prior surgery or

1463 scarring in the anterior neck, small goiter size, RAIU sufficient to allow therapy,

1464 and lack of access to a high-volume thyroid surgeon (the latter factor is more

1465 important for TMNG than for TA).

1466 b. Surgery: Presence of symptoms or signs of compression within the neck, concern

1467 for coexisting thyroid cancer, coexisting hyperparathyroidism requiring surgery, 

decreased life-expectancy, and not good candidates for ablative therapy.

\section{Contraindications to a particular modality as treatment for TMNG or TA:}

1473

a. RAI therapy: Definite contraindications to the use of radioactive iodine include pregnancy, lactation, coexisting thyroid cancer, individuals unable to comply with radiation safety guidelines and used with caution in women planning a pregnancy within 4-6 months.

b. Surgery: Factors weighing against the choice of surgery include significant comorbidity such as cardiopulmonary disease, end-stage cancer, or other debilitating disorders, or lack of access to a high volume thyroid surgeon. Pregnancy is a relative contraindication and should only be used in this circumstance when rapid control of hyperthyroidism is required and ATDs cannot be used. Thyroidectomy is best avoided in the first and third trimesters of pregnancy because of teratogenic effects associated with anesthetic agents and increased risk of fetal loss in the first trimester, and increased risk of preterm labor in the third. Optimally, thyroidectomy should be performed in the latter portion of the second trimester. Although it is the safest time, it is not without risk $(4.5 \%-5.5 \%$ risk of preterm labor) $(67,68)$.

c. Definite contraindications to ATD therapy include previous known major adverse reactions to ATDs. 


\section{Factors that may impact patient preference:}

a. RAI therapy: Patients with either TMNG or TA choosing RAI therapy would likely place relatively higher value on the avoidance of surgery and attendant hospitalization or complications arising from either surgery or anesthesia; also, patients with TMNG would place greater value on the possibility of remaining euthyroid after RAI treatment.

b. Surgery: Patients choosing surgery would likely place a relatively higher value on definitive control of hyperthyroid symptoms, avoidance of exposure to radioactivity and a lower value on potential surgical and anesthetic risks; patients with TMNG choosing surgery would place a lower value on the certain need for lifelong thyroid hormone replacement whereas patients with TA who choose avoidance of exposure to radioactivity and on potential surgical and anesthetic risks and a lower value on the certain need for lifelong thyroid ATD therapy. 
1510 Because RAI treatment of TMNG or TA can cause a transient exacerbation of

1511 hyperthyroidism, beta-adrenergic blockade should be considered even in

1512 asymptomatic patients who are at increased risk for complications due to worsening of

1513 hyperthyroidism, i.e. elderly patients and patients with co-morbidities. Weak

1514 recommendation, low-quality evidence.

1515 Medical management before RAI therapy should be tailored to the patient's

1516 risk for complications if hyperthyroidism worsens, based on the severity of the

1517 hyperthyroidism, patient age, and comorbid conditions. Worsened chemical

1518 hyperthyroidism with increased heart rate and rare cases of supraventricular

1519 tachycardia, including atrial fibrillation and atrial flutter, have been observed in patients

1520 treated with RAI for either TMNG or nontoxic multindoular goiter (MNG) (267-269).

1521 In susceptible patients with pre-existing cardiac disease or in the elderly, this may

1522 produce significant clinical worsening (268). Therefore, the use of beta-blockers to

1523 prevent post-treatment tachyarrhythmias should be considered in all patients with

1524 TMNG or TA who are older than 60 years of age and those with cardiovascular disease

1525 or severe hyperthyroidism (31). The decision regarding the use of MMI pretreatment is

1526 more complex and is discussed below.

1527 - RECOMMENDATION 39

1528 In addition to beta-adrenergic blockade (see Recommendation 2 and 38) pretreatment

1529 with MMI prior to RAI therapy for TMNG or TA should be considered in patients

1530 who are at increased risk for complications due to worsening of hyperthyroidism, 
1531 including the elderly and those with cardiovascular disease or severe hyperthyroidism.

1532 Weak recommendation, low-quality evidence.

1534 In patients who are at increased risk for complications due to worsening of

1535 hyperthyroidism, resuming ATDs 3-7 days after RAI administration should be

1536 considered. Weak recommendation, low quality evidence.

1537 Young and middle-aged patients with TMNG or TA generally do not require

1538 pretreatment with ATDs (MMI) before receiving RAI, but may benefit from beta-

1539 blockade if symptoms warrant and contraindications do not exist.

Technical remarks: If an ATD is used in preparation for RAI therapy in patients

1541 with TMNG or TA, caution should be taken to avoid RAI therapy when the TSH is

1542 normal or elevated to prevent direct RAI treatment of perinodular and contralateral

1543 normal thyroid tissue, which increases the risk of developing hypothyroidism. However,

1544 if volume reduction is a goal, at the expense of an increased risk of hypothyroidism,

1545 pretreatment with MMI, allowing the TSH to rise slighty prior to RAI administration,

1546 results in greater volume reduction after fixed doses of RAI (270). Similarly, a recent

1547 meta-analysis indicated that the application of rhTSH before RAI therapy in non-toxic or

1548 TMNG results in greater thyroid volume reduction but higher hypothyroidism rates than

1549 RAI therapy alone (271). Unless volume reduction is an important goal, rhTSH

1550 administration before RAI therapy of TMNG is not generally recommended as it could 
1551 possibly exacerbate hyperthyroidism (272), it represents an off-label use, and mainly

1552 stimulates RAI uptake in TSH-sensitive perinodular tissues (273).

1553 [K2] Evaluation of thyroid nodules before RAI therapy

1554 - RECOMMENDATION 41

1555 Nonfunctioning nodules on radionuclide scintigraphy or nodules with suspicious

1556 ultrasound characteristics should be managed according to published guidelines

1557 regarding thyroid nodules in euthyroid individuals. Strong recommendation,

1558 moderate-quality evidence.

1559 Thorough assessment of suspicious nodules within a TMNG, according to the 1560 published guidelines $(225,274)$, should be completed before selection of RAI as the 1561 treatment of choice. The prevalence of thyroid cancer in TMNG historically has been 1562 estimated to be about 3\% (247). More recently, it has been estimated to be as high as $15639 \%$, which is similar to the $10.6 \%$ prevalence noted in nontoxic MNG (275).

1564 Technical remarks: Both the ATA and AACE, the latter in conjunction with the

1565 European Thyroid Association and Associazione Medici Endocrinologi, and the Latin

1566 American Thyroid Society have published management guidelines for patients with 1567 thyroid nodules $(225,274,276,277)$.

1568 [K3] Administration of RAI in the treatment of TMNG or TA 
1570 Sufficient activity of RAI should be administered in a single application to alleviate

1571 hyperthyroidism in patients with TMNG. Strong recommendation, moderate-

1572 quality evidence.

1573 The goal of RAI therapy, especially in older patients, is elimination of the

1574 hyperthyroid state. Higher activities of RAI, even when appropriately calculated for the

1575 specific volume or mass of hyperthyroid tissue, result in more rapid resolution of

1576 hyperthyroidism and less need for retreatment, but a higher risk for early

1577 hypothyroidism. One study showed a $64 \%$ prevalence of hypothyroidism 24 years after

1578 RAI therapy for TMNG, with a higher prevalence among patients who required more

1579 than one treatment (250). The prevalence of hypothyroidism following RAI therapy is

1580 increased by normalization or elevation of TSH at the time of treatment resulting from

1581 ATD pretreatment or use of rhTSH, and by the presence of antithyroid antibodies (278).

1582 The activity of RAI used to treat TMNG, calculated on the basis of goiter size to 1583 deliver 150-200 $\mu \mathrm{Ci}$ (5.55-7.4 MBq) per gram of tissue corrected for 24-hour RAIU, is 1584 usually higher than that needed to treat GD. In addition, the RAIU values for TMNG may

1585 be lower, necessitating an increase in the applied activity of RAI. Radiation safety

1586 precautions may be onerous if high activities of RAI are needed for large goiters. Both

1587 pretreatment with MMI allowing the TSH to rise slightly (270), or the off-label use of

1588 rhTSH (271), may reduce the total activity of RAI needed, but increase the risk of

1589 hypothyroidism (see prior discussion section [K1]).

Technical remarks: Enlargement of the thyroid is very rare after RAI treatment.

1591 However, patients should be advised to immediately report any tightening of the neck, 
1592 difficulty breathing, or stridor following the administration of RAI. Any compressive

1593 symptoms, such as discomfort, swelling, dysphagia, or hoarseness, which develop

1594 following RAI therapy, should be carefully assessed and monitored, and if clinically

1595 necessary, corticosteroids can be administered. Respiratory compromise in this setting is

1596 extremely rare and requires management as any other cause of acute tracheal

1597 compression.

- RECOMMENDATION 43

1599 Sufficient activity of RAI should be administered in a single application to alleviate

1600 hyperthyroidism in patients with TA. Strong recommendation, moderate-quality $1601 \quad$ evidence.

RAI administered to treat TA can be given either as a fixed activity of

1603 approximately $10-20 \mathrm{mCi}(370-740 \mathrm{MBq})$ or an activity calculated on the basis of nodule

1604 size using 150-200 $\mu \mathrm{Ci}$ (5.5-7.4 MBq) RAI per gram corrected for 24-hour RAIU (278).

1605 A long-term follow-up study of patients with TA, where patients with nodules $<4 \mathrm{~cm}$

1606 were administered an average of $13 \mathrm{mCi}(481 \mathrm{MBq})$ and those with larger nodules an

1607 average of $17 \mathrm{mCi}(629 \mathrm{MBq})$, showed a progressive increase in hypothyroidism over

1608 time in both groups, suggesting that hypothyroidism develops over time regardless of

1609 activity adjustment for nodule size (259). A randomized trial of 97 patients with TA

1610 compared the effects of high $(22.5 \mathrm{mCi} / 833 \mathrm{MBq})$ or low $(13 \mathrm{mCi} / 481 \mathrm{MBq})$ fixed

1611 activity RAI, with a calculated activity that was either high (180-200 $\mu \mathrm{Ci} / \mathrm{g} / 6.7-7.4 \mathrm{~Bq})$

1612 or low (90-100 $\mu \mathrm{Ci} / \mathrm{g} / 3.3-3.7 \mathrm{~Bq})$ and corrected for 24-hour RAIU (279). This study

1613 confirmed previous reports showing an earlier disappearance of hyperthyroidism and 
1614 earlier appearance of hypothyroidism with higher RAI activity. Use of a calculated

1615 activity allowed for a lower RAI activity to be administered for a similar efficacy in the

1616 cure of hyperthyroidism.

1617 [K4] Patient follow-up after RAI therapy for TMNG or TA

1618 - RECOMMENDATION 44

1619 Follow-up within the first 1-2 months after RAI therapy for TMNG or TA should

1620 include an assessment of free $\mathrm{T}_{4}$, total $\mathrm{T}_{3}$ and TSH. Biochemical monitoring should be

1621 continued at 4-6 week intervals for 6 months, or until the patient becomes

1622 hypothyroid and is stable on thyroid hormone replacement. Strong recommendation,

$1623 \quad$ low-quality evidence.

1624 RAI therapy for TMNG results in resolution of hyperthyroidism in approximately

$162555 \%$ of patients at 3 months and $80 \%$ of patients at 6 months, with an average failure rate

1626 of $15 \%$ (246-248). Goiter volume is decreased by 3 months, with further reduction

1627 observed over 24 months, for a total size reduction of $40 \%$ (248). For TA, $75 \%$ of

1628 patients were no longer hyperthyroid at 3 months, with nodule volume decreased by $35 \%$

1629 at 3 months and by $45 \%$ at 2 years (257). Risk of persistent or recurrent hyperthyroidism

1630 ranged from $0 \%$ to $30 \%$, depending on the series $(246-248,257)$. Long-term follow-up

1631 studies show a progressive risk of clinical or subclinical hypothyroidism of about $8 \%$ by

16321 year and $60 \%$ by 20 years for TA (259), and an average of 3\% by 1 year and $64 \%$ by 24

1633 years for TMNG (250). 
1635 Such patients develop worsening hyperthyroidism within a few months of RAI therapy.

1636 Treatment with additional RAI is effective.

1637 Technical remarks: If thyroid hormone therapy is necessary, the dose required

1638 may be less than full replacement due to underlying persistent autonomous thyroid

1639 function.

1640 [K5] Treatment of persistent or recurrent hyperthyroidism following RAI therapy for

1641 TMNG or TA

1642 - RECOMMENDATION 45

1643 If hyperthyroidism persists beyond 6 months following RAI therapy for TMNG or

1644 TA, retreatment with RAI is suggested. In selected patients with minimal response 3

1645 months after therapy additional RAI may be considered. Weak recommandation,

$1646 \quad$ low-quality evidence.

1647 Technical remarks: In severe or refractory cases of persistent hyperthyroidism

1648 due to TMNG or TA, following treatment with RAI, surgery may be considered. As

1649 some patients with mild hyperthyroidism following RAI administration will continue to

1650 improve over time, use of MMI with close monitoring may be considered to allow

1651 control of the hyperthyroidism until the RAI is effective. 


\section{- RECOMMENDATION 46}

1656 If surgery is chosen as treatment for TMNG or TA, patients with overt

1657 hyperthyroidism should be rendered euthyroid prior to the procedure with MMI

1658 pretreatment, with or without beta-adrenergic blockade. Preoperative iodine should not

1659 be used in this setting. Strong recommendation, low-quality evidence.

1660 Risks of surgery are increased in the presence of thyrotoxicosis. Thyrotoxic crisis

1661 during or after the operation, can result in extreme hypermetabolism, hyperthermia,

1662 tachycardia, hypertension, coma, or death. Therefore, prevention with careful preparation

1663 of the patient is of paramount importance $(281,282)$. The literature reports a very low risk

1664 of anesthesia-related mortality associated with thyroidectomy $(254,283)$. Preoperative

1665 iodine therapy is not indicated due to the risk of exacerbating the hyperthyroidism (284).

1666 Usually hyperthyroidism is less severe in patients with TMNG, so that in most cases,

1667 patients with allergy to ATDs can be prepared for surgery, when necessary, with beta1668 blockers alone.

1669 [L2] The surgical procedure and choice of surgeon

1670 - RECOMMENDATION 47

1671 If surgery is chosen as treatment for TMNG, near-total or total thyroidectomy should 1672 be performed. Strong recommendation, moderate-quality evidence. 
1674 performed initially (285). This procedure can be performed with the same low rate of

1675 complications as a subtotal thyroidectomy (286-289). Reoperation for recurrent or

1676 persistant goiter results in a 3- to 10-fold increase in the risk of permanent vocal cord

1677 paralysis or hypoparathyroidism $(290,291)$.

1678 - RECOMMENDATION 48

1679 Surgery for TMNG should be performed by a high-volume thyroid surgeon. Strong 1680 recommendation, moderate-quality evidence.

TMNG is more common in older patients. Data regarding outcomes following

1682 thyroidectomy in elderly patients have shown conflicting results. Overall, however,

1683 studies conducted at the population level have demonstrated significantly higher rates

1684 of postoperative complications, longer length of hospital stay, and higher costs among

1685 elderly patients (198). Data showing equivalent outcomes among the elderly usually

1686 have come from high-volume centers (292). There are robust data demonstrating that

1687 surgeon volume of thyroidectomies is an independent predictor of patient clinical and

1688 economic outcomes (i.e., in-hospital complications, length of stay, and total hospital

1689 charges) following thyroid surgery $(198,199,293)$. The recommendation for referral to a

1690 high-volume surgeon is essentially the same as that described in section [F2] for the

1691 choice of surgeon in GD. 
1693 If surgery is chosen as the treatment for TA, a thyroid ultrasound should be done to

1694 evalutate the entire thyroid gland. An ipsilateral thyroid lobectomy, or isthmusectomy

1695 if the adenoma is in the thyroid isthmus, should be performed for isolated TAs. Strong

1696 recommendation, moderate-quality evidence.

A preoperative thyroid ultrasound is useful, as it will detect the presence of

1698 contralateral nodularity that is suspicious in appearance or that will necessitate future

1699 surveillance, both circumstances in which a total thyroidectomy may be more

1700 appropriate. Lobectomy removes the TA while leaving normal thyroid tissue, allowing

1701 residual normal thyroid function in the majority of patients. One large clinical series for

1702 TA demonstrated no surgical deaths and low complication rates (254). In patients who

1703 wish to avoid general anesthesia or who have significant comorbidities, the risk of

1704 anesthesia can be lowered further when cervical block analgesia with sedation is

1705 employed by thyroid surgeons and anesthesiologists experienced in this approach (294).

1706 Patients with positive antithyroid antibodies preoperatively have a higher risk of

1707 postoperative hypothyroidism $(256,278)$.

1708 - RECOMMENDATION 50

1709 We suggest that surgery for TA be performed by a high-volume surgeon. Weak

1710 recommendation, moderate-quality evidence.

1711 While surgeon experience in the setting of TA is of somewhat less importance

1712 than in TMNG, it remains a factor to consider in deciding between surgery and RAI

1713 therapy. High-volume thyroid surgeons tend to have better outcomes following 
1714 lobectomy than low-volume surgeons, but the differences are not statistically

1715 significant (198). High-volume surgeons may be more comfortable with performing the

1716 thyroid lobectomy under cervical block analgesia with sedation.

1717 [L3] Postoperative care

1718 - RECOMMENDATION 51

1719 Following thyroidectomy for TMNG, serum calcium \pm iPTH levels should be

1720 measured, and oral calcium and calcitriol supplementation administered based on the

1721 results. Weak recommendation, low-quality evidence.

1722 Technical remarks: The management of hypocalcemia following thyroidectomy

1723 for TMNG is essentially the same as that described in section [F3] for postoperative

1724 management in GD. Severe or prolonged preoperative hyperthyroidism, and larger size

1725 and greater vascularity of the goiter (more typically seen in GD) increases the risk of

1726 postoperative hypocalcemia.

1727 - RECOMMENDATION 52

1728 MMI should be stopped at the time of surgery for TMNG or TA. Beta-adrenergic

1729 blockade should be slowly discontinued following surgery. Strong recomendation,

$1730 \quad$ low-quality evidence.

Technical remarks: The duration over which beta-adrenergic blockade should

1732 be tapered should take into account the preoperative free $\mathbf{T}_{4}$ concentration, the 
1733 heart rate, and the week-long half-life of $\mathbf{T}_{\mathbf{4}}$. Additionally, patients taking

1734 higher doses of beta-blockers will require a longer taper.

1736 Following thyroidectomy for TMNG, thyroid hormone replacement should be started

1737 at a dose appropriate for the patient's weight $(0.8 \mathrm{mcg} / \mathrm{lb}$ or $1.6 \mathrm{mcg} / \mathrm{kg})$ and age, with

1738 elderly patients needing somewhat less. TSH should be measured every 1-2 months

1739 until stable, and then annually. Strong recommendation, low-quality evidence.

1740 Technical remarks: The appropriate dosing of L-thyroxine will vary with patient

1741 BMI (219). If a significant thyroid remnant remains following thyroidectomy,

1742 because such a remnant may demonstrate autonomous production of thyroid

1743 hormone, immediate postoperative doses of thyroid hormone should be initiated at

1744 somewhat less than full replacement doses and subsequently adjusted based on

1745 thyroid function testing.

1746 - RECOMMENDATION 54

1747 Following lobectomy for TA, TSH and estimated free $\mathrm{T}_{4}$ levels should be obtained 4-

17486 weeks after surgery, and thyroid hormone supplementation started if there is a

1749 persistent rise in TSH above the normal range. Strong recommendation, low-quality

$1750 \quad$ evidence.

1751

Technical remarks: After lobectomy for TA, serum calcium levels do not need to

1752 be obtained, and calcium and calcitriol supplements do not need to be administered. 
1753 Thyroid hormone replacement is required in about $15-20 \%$ of patients following thyroid

1754 lobectomy (295). Serum TSH levels may have been suppressed or normal prior to

1755 lobectomy, depending on the degree of preoperative preparation with ATDs. TSH levels

1756 may remain in the high normal range for 3-6 months following lobectomy; therefore,

1757 continued monitoring in an asymptomatic patient for 4-6 months postoperatively is

1758 reasonable, since there may be eventual recovery of normal thyroid function (296).

1759 [L4] Treatment of persistent or recurrent disease following surgery for TMNG or TA

1760 - RECOMMENDATION 55

1761 RAI therapy should be used for retreatment of persistent or recurrent hyperthyroidism

1762 following inadequate surgery for TMNG or TA. Strong recommendation, low-

1763 quality evidence.

1764 Persistent or recurrent hyperthyroidism following surgery is indicative of

1765 inadequate surgery. As remedial thyroid surgery comes at significantly increased risk of

1766 hypoparathyroidism and RLN injury, it should be avoided, if possible, in favor of RAI

1767 therapy $(290,291)$. If this is not an option, it is essential that the surgery be performed by

1768 a high-volume thyroid surgeon.

1769

1770 [M] If ATDs are chosen as treatment of TMNG or TA, how should the therapy be

$1771 \quad$ managed? 
1773 discontinuation of treatment results in relapse $(262,297)$. However, prolonged (life-long)

1774 ATD therapy may be the best choice for some individuals with limited life-expectancy

1775 and increased surgical risk, including residents of nursing homes or other care facilities

1776 where compliance with radiation safety regulations may be difficult.

1777 — RECOMMENDATION 56

1778 Long-term MMI treatment of TMNG or TA might be indicated in some elderly or

1779 otherwise ill patients with limited life-expectancy, in patients who are not good

1780 candidates for surgery or ablative therapy, and in patients who prefer this option.

1781 Weak recommendation, low-quality evidence.

1783 Technical remarks: The required dose of MMI to restore the euthyroid state in

1784 TMNG or TA patients is usually low (5-10 mg/day). Because long-term, low-dose ATD

1785 treatment in nodular hyperthyroidism can be difficult to regulate, frequent (every 3

1786 months) monitoring is recommended initially, especially in the elderly (298), until

1787 stability has been documented after which testing frequency can be decreased.

1788

1789 [N] Is there a role for ethanol or radiofrequency ablation in the management of TA or $1790 \quad$ TMNG? 
1792 Alternative therapies such as ethanol or radiofrequency ablation of TA and TMNG can

1793 be considered in select patients where RAI, surgery or long-term ATD are

1794 inappropriate, contraindicated, or refused, and expertise in these procedures is

1795 available. No recommendation, insufficient evidence to assess benefits and risks.

$1796 \quad[N 1]$ Ethanol ablation

Reports that support the efficacy of percutaneous ethanol injection (PEI) under

1798 sonographic guidance to treat TA and TMNG come largely from Europe (299-301).

1799 Experience in the United States is limited. A typical protocol involves the injection of

1800 ethanol (average dose $10 \mathrm{ml}$, depending on size of the area to be ablated) into the TA or

1801 autonomous area of a TMNG. In one study, the average patient required 4 sessions at 2

1802 week intervals (299). One hundred twenty-five patients with TA were followed for an

1803 average of 5 years; $2.4 \%$ refused further treatment due to pain, and $3.2 \%$ had

1804 complications including transient recurrent laryngeal nerve palsy, abscess or hematoma

1805 (299). Ninety-three percent of patients achieved a functional cure (no uptake on RAI

1806 scintigraphy), and $92 \%$ had a $>50 \%$ reduction in nodule size (299). In another study of

1807 both TA and TMNG, 78 percent achieved a functional cure, all nodules regressed, and

1808 there was no recurrent hyperthyroidism during 5-years of follow-up (300). Ethanol

1809 ablation also has been used following RAI to reduce nodule size (301). However, its use

1810 has been limited due to pain associated with extravasation of the ethanol to extranodular

1811 locations, and other adverse effects which have included transient thyrotoxicosis,

1812 permanent ipsilateral facial dysethesia, paranodular fibrosis interfering with subsequent

1813 surgery (302), and toxic necrosis of the larynx and adjacent skin (303). 
1815 Both radiofrequency ablation (RFA) and laser therapy have been used to treat 1816 thyroid nodules. A meta-analysis demonstrated that RFA resulted in larger reductions in 1817 nodule size with fewer sessions than laser (304). A retrospective multi-center report of 1818 RFA for TA in 44 patients utilized an $18 \mathrm{~g}$ electrode under ultrasound guidance with a 1819 mean follow-up of 20 months (305). An 82\% reduction in nodule volume was achieved, 1820 but $20 \%$ of nodules remained autonomous on scintigraphy, and $18 \%$ of patients remained 1821 hyperthyroid. All patients complained of pain during the procedure, but there were no 1822 complications (305). A Korean study compared the use of RFA to surgery for non-toxic 1823 nodules (306). RFA was associated with an $85 \%$ reduction in nodule size, the cost was

1824 similar to surgery, there were fewer complications (recurrent laryngeal nerve injury or 1825 hypoparathyroidism: $6 \%$ for surgery and $1 \%$ for RFA), and no patient who received RFA 1826 became hypothyroid (306). Advocates of RFA argue that it preserves normal thyroid 1827 function compared to surgery or RAI (307). However, additional data are needed to 1828 determine the success at correcting hyperthyroidism in patients with TA and TMNG. The 1829 use of RFA should be limited to centers where clinicians have received adequate training 1830 in the technique.

$1833 \quad[\mathrm{O} 1]$ General approach 
1835 Children with GD should be treated with MMI, RAI therapy, or thyroidectomy. RAI

1836 therapy should be avoided in very young children ( $<5$ years). RAI therapy in children

1837 is acceptable if the activity is $>150 \mu \mathrm{Ci} / \mathrm{g}(5.55 \mathrm{MBq} / \mathrm{g})$ of thyroid tissue, and for

1838 children between 5 and 10 years of age if the calculated RAI administered activity is

$1839<10 \mathrm{mCi}(<473 \mathrm{MBq})$. Thyroidectomy should be chosen when definitive therapy is

1840 required, the child is too young for RAI, and surgery can be performed by a high-

1841 volume thyroid surgeon. Strong recommendation, moderate-quality evidence.

1842 The treatment of pediatric patients with GD varies considerably among

1843 institutions and practitioners. It is important to recognize that lasting remission after

1844 ATD therapy occurs in only a minority of pediatric patients with GD, including

1845 children treated with ATDs for many years. In determining the initial treatment

1846 approach, the patient's age, clinical status, and likelihood of remission should be

1847 considered. Patient and parent values and preferences should also be strongly

1848 considered when choosing one of the three treatment modalities.

1849 Because some children will go into remission, MMI therapy for 1 year is still

1850 considered first-line treatment for most children. However, the majority of pediatric

1851 patients with GD will eventually require either RAI or surgery. When ATDs are used in

1852 children, only MMI should be used, except in exceptional circumstances. If clinical

1853 characteristics suggest a low chance of remission at initial presentation (see [P6] below)

1854 MMI, RAI, or surgery may be considered initially. If remission is not achieved after a

1855 course of therapy with ATDs, RAI or surgery should be considered. Alternatively, MMI 
1856 therapy may be continued long-term, or until the child is considered old enough for 1857 surgery or RAI.

1858 Properly administered, RAI is an effective treatment for GD in the pediatric 1859 population (308-310). RAI is widely used in children, but still viewed as controversial by 1860 some practitioners owing primarily to concern over cancer risks $(311,312)$. Although 1861 there are sparse clinical data relating to RAI use in children with GD and subsequent 1862 thyroid cancer (313), it is known that risks of thyroid cancer after external irradiation are 1863 highest in children $<5$ years of age, and they decline with advancing age $(314,315)$; see 1864 discussion of RAI therapy and cancer risk in [P3] below. In comparison, activities of RAI 1865 used with contemporary therapy are not known to be associated with an increased risk of 1866 thyroid neoplasm in children.

1867 Thyroidectomy is an effective treatment for GD, but is associated with a higher 1868 complication rate in children than adults (316-318). Thyroidectomy should be performed 1869 in those children who are too young for RAI, provided that surgery can be performed by 1870 a high-volume thyroid surgeon, preferably with experience in conducting thyroidectomies 1871 in children. Technical remarks: There may be circumstances in which RAI therapy is 1873 indicated in young children, such as when a child has developed a reaction to ATDs, 1874 proper surgical expertise is not available, or the patient is not a suitable surgical 1875 candidate. 

recommendation, moderate-quality evidence.

1884 daily, even in patients with severe hyperthyroidism. Although many practitioners give 1885 MMI in divided doses, data in adults do not support a need for such and show that 1886 compliance with once-daily MMI therapy is superior to multiple daily doses of PTU 1887 (83\% vs. 53\%) (319). The MMI dose typically used is $0.2-0.5 \mathrm{mg} / \mathrm{kg}$ daily, with a range 1888 from $0.1-1.0 \mathrm{mg} / \mathrm{kg}$ daily (320-322). One approach is to prescribe the following whole 1889 tablet or quarter to half-tablet doses: infants, $1.25 \mathrm{mg} /$ day; $1-5$ years, $2.5-5.0 \mathrm{mg} /$ day; 5189010 years, 5-10 mg/day; and 10-18 years, 10-20 mg/day. With severe clinical or 1891 biochemical hyperthyroidism, doses that are 50-100\% higher than the above can be used. 
1898 more to maintain a euthyroid state (112). Alternatively, some physicians elect not to

1899 reduce the MMI dose and add levothyroxine to make the patient euthyroid, a practice

1900 referred to as “block and replace.” However, because meta-analyses suggest a higher

1901 prevalence of adverse events using block-and-replace regimens than dose titration

1902 (119,323), likely due to higher doses of MMI and the dose-related complications

1903 associated with MMI (324), we suggest that this practice be avoided. However, it may

1904 have utility in rare patients, after addressing compliance, who are inadequately

1905 controlled on one dose of MMI, then become hypothyroid after a minimal dose

1906 increase.

Practitioners should also monitor the weight of children treated with ATDs.

1908 Excessive weight gain within 6 months of treatment is seen in children treated for GD,

1909 and the gain in weight can persist (325). Parents and patients should be counseled about

1910 this possibility and nutrition consultation considered if excessive weight gain occurs.

\section{2 — RECOMMENDATION 60}

1913 Pediatric patients and their caretakers should be informed of side effects of ATD

1914 preferably in writing, and the necessity of stopping the medication immediately and

1915 informing their physician if they develop pruritic rash, jaundice, acolic stools or dark

1916 urine, arthralgias, abdominal pain, nausea, fatigue, fever, or pharyngitis. Strong

1917 recommendation, low-quality evidence. 
1919 Prior to initiating ATD therapy, we suggest that pediatric patients have, as a baseline, 1920 complete blood cell count, including white blood cell count with differential, and a 1921 liver profile including bilirubin, transaminases, and alkaline phosphatase. Weak 1922 recommendation, low-quality evidence. PTU is associated with an unacceptable risk of hepatotoxicity in children, with a 1924 risk of liver failure of 1 in $2000-4000$ children taking the medication (326,327). PTU can 1925 cause fulminant hepatic necrosis that may be fatal; liver transplantation has been 1926 necessary in some patients taking PTU (326). It is for this reason that the FDA issued a 1927 black box warning regarding the use of PTU (328), noting that 32 (22 adult and 10 1928 pediatric) cases of serious liver injury have been associated with PTU use $(326,328)$. 1929 Furthermore, since the recommendation was issued that PTU use in children be avoided, 1930 we are unaware of any published cases of PTU-related liver failure (327).

1931 Because PTU-induced liver injury is of rapid onset and can be rapidly

1932 progressive, biochemical monitoring of liver function tests and transaminase levels has 1933 not been shown to be useful in surveillance for PTU-related liver injury. When neither 1934 prompt surgery nor RAI therapy are options, and ATD therapy is necessary in a patient 1935 who has developed a minor toxic reaction to MMI, a short course of PTU use can be 1936 considered. When surgery is the planned therapy and MMI cannot be administered, if the 1937 patient is not too thyrotoxic (and the hyperthyroidism is due to GD), the hyperthyroid 1938 state can be controlled before surgery with beta blockade and SSKI (50 mg iodide/ drop) $19393-7$ drops $(0.15-0.35 \mathrm{~mL})$ by mouth, given three times a day for 10 days before surgery. 
1940 Prior to surgery it is desirable to have the free $T_{4}$, or total $T_{4}$ and total $T_{3}$ levels in the

1941 normal or subnormal range. Alternatively, if the surgery cannot be performed within a

1942 few weeks, a short course of PTU may be administered with the child closely monitored

1943 clinically for signs of hepatic dysfunction including nausea, anorexia, malaise and

1944 abdominal pain.

MMI may also be associated with hepatotoxicity in children, but this tends to be

1946 milder and is typically cholestatic rather than hepatocellular (326). There is at least one

1947 reported case of cholestatic jaundice in a child (326). However, there have been reports of

1948 hepatocellular toxicity with MMI in adults (134).

1949 MMI may also be associated with antineutrophil cytoplasmic antibody (ANCA)

1950 positive vasculitis (329), although this occurs far less frequently than with PTU. Patients

1951 of Asian origin seem to be more susceptible to this adverse reaction, and it can develop

1952 after months to years of therapy. Many PTU-treated patients also develop ANCA

1953 positivity on treatment, but remain asymptomatic (330). Typical manifestations of

1954 ANCA positive vasculitis are: polyarthritis, purpuric skin lesions, and occasionally

1955 pulmonary and/or renal involvement. Discontinuation of the drug generally results in

1956 resolution of the symptoms, but in more severe cases, glucocorticoids or other

1957 immunosuppressive therapy may be needed.

Technical remarks: It is advisable to provide information concerning side effects

1959 of ATDs to the patient or caretaker in writing. See Recommendation 14 Technical

1960 remarks for a discussion regarding the utility of obtaining complete blood count and liver

1961 profile before initiating MMI therapy. 


\section{- RECOMMENDATION 62}

1964 Beta adrenergic blockade is recommended for children experiencing symptoms of 1965 hyperthyroidism, especially those with heart rates in excess of 100 beats per minute.

1966 Strong recommendation, low-quality evidence.

1967 In children in whom the diagnosis of Graves' hyperthyroidism is strongly

1968 suspected or confirmed, and who are showing significant symptoms, including, but not

1969 limited to, tachycardia, muscle weakness, tremor, or neuropsychological changes,

1970 treatment with atenolol, propranolol, metoprolol, or other beta-blockers leads to a

1971 decrease in heart rate and symptoms of GD. In those with reactive airway disease, cardio

1972 selective beta-blockers such as atenolol or metoprolol can be used cautiously (331), with

1973 the patient monitored for exacerbation of asthma.

1974 [P3] Monitoring of children taking MMI

1976 obtained at 2-6 weeks, the dose is adjusted if indictated, and thyroid function tests are

1977 measured again at 4-6 weeks, and then every 2-3 months once the dose is stablized.

1978 Depending on the severity of hyperthyroidism and the MMI dose, it can take several

1979 months for elevated thyroid hormone levels to fall into the normal range. Serum TSH

1980 may remain suppressed for several months after starting therapy and is therefore not a

1981 good parameter to monitor therapy early in the course. 
1983 ATDs should be stopped immediately, and white blood counts measured in children 1984 who develop fever, arthralgias, mouth sores, pharyngitis, or malaise. Strong 1985 recommendation, low-quality evidence. Although MMI has a better overall safety profile than PTU, MMI is associated 1987 with minor adverse events that may affect up to $20 \%$ of children (332). MMI-related 1988 adverse events include allergic reactions, rashes, myalgias, and arthralgias $(333,334)$, as 1989 well as hypothyroidism from overtreatment. Side effects from MMI usually occur within 1990 the first 3 months of starting therapy, but adverse events can occur later. In children, the 1991 risks of MMI-related cholestasis and hepatocellular injury appear to be much less than 1992 that observed in adults (326).

1994 PTU (128,324,335). Data on the prevalence of agranulocytosis in children are 1995 unavailable, but it is estimated to be very low. In adults, agranulocytosis is dose 1996 dependent with MMI, and rarely occurs at low doses (e.g., 5-10 mg/day) (128,324,335). 1997 When agranulocytosis develops, $95 \%$ of the time it occurs in the first 100 days of therapy $1998(128,324,335)$. The overall rate of side effects from ATDs (both major and minor) in 1999 children has been reported to be $6 \%-35 \%(332,334,336,337)$. Technical remarks: While routine monitoring of white blood counts may 2001 occasionally detect early agranulocytosis, it is not recommended because of the rarity of 2002 the condition and its sudden onset, which is generally symptomatic. It is for this reason 
2003 that measuring white cell counts during febrile illnesses and at the onset of pharyngitis 2004 has become the standard approach for monitoring.

2005 [P4] Monitoring of children taking PTU

2006 RECOMMENDATION 64

2007 In general, PTU should not be used in children. But, if used the medication should be 2008 stopped immediately and liver function and hepatocellular integrity assessed in 2009 children who experience anorexia, pruritus, rash, jaundice, light-colored stool or dark 2010 urine, joint pain, right upper quadrant pain or abdominal bloating, nausea, or malaise.

2011 Strong recommendation, low-quality evidence.

2013 in symptomatic patients or found incidentally) reach 2-3 times the upper limit of normal.

2014 After discontinuing the drug, liver function tests (i.e., bilirubin, alkaline phosphatase, and 2015 transaminases) should be monitored weekly until there is evidence of resolution. If there 2016 is no evidence of resolution, referral to a gastroenterologist or hepatologist is warranted.

2017 [P5] Management of allergic reactions in children taking MMI

2018 - RECOMMENDATION 65

2019 Persistent minor cutaneous reactions to MMI therapy in children should be managed

2020 by concurrent antihistamine treatment or cessation of the medication and changing to 2021 therapy with RAI or surgery. In the case of a serious adverse reaction to an ATD, 

quality evidence.

If children develop serious adverse reactions to MMI, RAI or surgery should be

2025 considered because the risks of PTU are viewed to be greater than the risks of radioactive

2026 iodine or surgery. In special circumstances, where the patient is viewed to be at risk for

2027 thyroid storm and ATD therapy is needed in a child with a serious adverse reaction to

2028 MMI, PTU may be considered for short-term therapy to control hyperthyroidism. In this

2029 setting, families should be informed of the risks of PTU.

2030 [P6] Duration of MMI therapy in children with GD

\section{- RECOMMENDATION 66}

2032 If MMI is chosen as the first-line treatment for GD in children, it may be tapered in 2033 those children requiring low doses after 1-2 years to determine if a spontaneous 2034 remission has occurred, or it may be continued until the child and caretakers are 2035 ready to consider definitive therapy, if needed. Strong recommendation, moderate2036 quality evidence. The issue of how long ATDs should be used in children before considering either

2038 RAI or surgery is a topic of controversy and warrants further study. Prospective studies in 2039 adults show that if remission does not occur after 12-18 months of therapy, there is a 2040 lower chance of remission occurring with prolonged therapy (338). In children, when 2041 ATDs are used for 1-2 years, remission rates are generally $20 \%-30 \%$, with remission 2042 defined as being euthyroid for 1 year after cessation of therapy $(333,339,340)$. 
2043 Retrospective studies have suggested that the chance of remission after 2 years of ATDs 2044 is low if the thyroid gland is large (more than 2.5 times normal size for age), the child is 2045 young $(<12$ years) or not Caucasian, serum TRAb levels are above normal on therapy, or 2046 free $\mathrm{T}_{4}$ are substantially elevated at diagnosis ( $\left.>4 \mathrm{ng} / \mathrm{dL} ; 50 \mathrm{pmol} / \mathrm{L}\right)$ (339). One

2047 prospective study suggested that likelihood of remission could best be predicted by the 2048 initial response to ATDs, with achievement of euthyroid state within 3 months, 2049 suggesting higher likelihood. Younger children and those with high initial thyroid 2050 hormone levels were also found to be less likely to achieve remission within 2 years in 2051 the prospective studies $(334,337)$.

Remission rates in children treated with ATDs for longer than 2 years have been 2053 reported. Although two decades ago it was suggested that $25 \%$ of children with GD go 2054 into remission with every 2 years of continued treatment (341), other studies of larger 2055 cohorts of pediatric patients with GD treated with ATDs for extended periods have not 2056 revealed similar remission rates $(333,339,342)$. Of 120 pediatric patients treated with 2057 ATDs at one center, after 1 year of therapy with ATDs, 25\% were in remission; after 2 2058 years, 26\%; after 4 years, 37\%; and after $4-10$ years, $15 \%$. Importantly, $30 \%$ of the 2059 children who went into remission eventually relapsed (333). In another large cohort of 2060184 medically treated children, after 1 year of therapy with ATDs, $10 \%$ were in 2061 remission; after 2 years, 14\%; after 3 years, 20\%; and after 4 years, $23 \%(339,342)$. More recently, in a retrospective analysis from Japan of 1,138 children, 723 were 2063 continued on long term ATD treatment, 271 underwent surgery or RAI, and 144 dropped 2064 out. Of the 639 patients of the 723 who discontinued ATD treatment, $46.2 \%$ achieved 
remission, and $34.2 \%$ relapsed. The prevalence of adverse events associated with MMI and PTU were $21.4 \%$ and $18.8 \%$, respectively (343).

2068 treated with ATDs are very low $(<20 \%)$, especially with longer follow-up, in cohorts

2069 from Germany (344) and Denmark (345).

2071 ATDs. In one study that compared outcomes of 32 prepubertal and 68 pubertal children, 2072 remission occurred in only $17 \%$ of prepubertal children treated $5.9 \pm 2.8$ years, compared 2073 with $30 \%$ of pubertal individuals treated $2.8 \pm 1.1$ years (340). In another report, the 2074 course of GD was compared in 7 prepubertal, 21 pubertal, and 12 postpubertal children 2075 (336). Remission was achieved in 10 patients (28\%) with similar rates among the three 2076 groups, whereas the time to remission tended to be longer in the small proportion of 2077 prepubertal children (median age, 6 years) (336).

Persistence of GD in children is correlated with the persistence of TRAbs. A

2079 recent study found that TRAb levels normalized after 24 months in only $18 \%$ of pediatric 2080 patients on ATDs (346). There were no data showing that there was normalization of 2081 TRAb levels when patients were on ATDs for a longer time. Therefore, it appears that 2082 TRAb levels persist longer in children than in adults (346). Whereas monitoring of TRAb 2083 levels while on ATDs has been shown to be useful in adult patients for predicting the 2084 likelihood of remission or relapse of GD after stopping the medication (172), this 2085 approach has yet to be validated in children. 
Whereas most studies, including recent large database reports (343), show that the

2087 vast majority of patients treated for GD with ATDs do not go into remission, a recent

2088 prospective report from France shows that with prolonged ATD use, remission rates of up

2089 to $\mathbf{4 9} \%$ could be achieved. This study reported remision rates of $20 \%, 37 \%, 45 \%$, and

$209049 \%$ after 4, 6, 8, and 10 years follow-up of 154 children treated with ATDs (337). The

2091 use of MMI in this group of children was associated with a very low rate of medication

2092 side-effects (337). Thus, whereas many practitioners will treat for 1-2 years with MMI,

2093 these data suggest that treatment for longer periods is also reasonable, as long as side-

2094 effects to medication do not occur.

\section{- RECOMMENDATION 67}

2096 Pediatric patients with GD who are not in remission following at least 1-2 years of

2097 MMI therapy should be considered for treatment with RAI or thyroidectomy.

2098 Alternatively, if children are tolerating ATD therapy, ATDs may be used for extended

2099 periods. This approach may be especially useful for the child not considered to be a

2100 candidate for either surgery or RAI. Individuals on prolonged ATDs therapy ( $>2$

2101 years) should be reevaluated every 6-12 months and when transitioning to adulthood.

2102 Strong recommendation, low-quality evidence.

If remission is not achieved upon stopping MMI after at least 1 or 2 years of

2104 therapy, RAI or surgery should be considered, depending on the age of the child.

2105 Alternatively, practitioners can continue MMI for extended periods, as long as adverse

2106 drug effects do not occur and the hyperthyroid state is controlled. As noted above,

2107 adverse reactions typically occur within the first few months of therapy. 
2109 [Q] If radioactive iodine is chosen as treatment for GD in children, how should it be

2110 accomplished?

2111 [Q1] Preparation of pediatric patients with GD for RAI therapy

2112 - RECOMMENDATION 68

2113 We suggest that children with GD having total $\mathrm{T}_{4}$ levels of $>20 \mathrm{ug} / \mathrm{dL}(260 \mathrm{nmol} / \mathrm{L})$ or

2114 free $\mathrm{T}_{4}>5 \mathrm{ng} / \mathrm{dL}(60 \mathrm{pmol} / \mathrm{L})$ who are to receive RAI therapy be pretreated with MMI

2115 and beta-adrenergic blockade until total $\mathrm{T}_{4}$ and/or free $\mathrm{T}_{4}$ normalize before proceeding

2116 with RAI treatment. Weak recommendation, low-quality evidence.

2117 Although the frequency of short-term worsening of hyperthyroidism following

2118 pretreatment with ATD therapy is not known, there are rare reports of pediatric patients

2119 with severe hyperthyroidism who have developed thyroid storm after receiving RAI

$2120(347,348)$.

Technical remarks: When children receiving MMI are to be treated with RAI, the

2122 medication should be stopped 2-3 days before treatment (349). At that time patients

2123 should be placed on beta-blockers (if not already taking) until total $\mathrm{T}_{4}$ and/or free $\mathrm{T}_{4}$

2124 levels normalize following RAI therapy, which generally takes 2-4 months. Although

2125 some physicians restart ATDs after treatment with RAI (80), this practice is seldom

2126 required in children $(309,310,350)$. Thyroid hormone levels in children begin to fall

2127 within the first week following RAI therapy. ATDs can complicate assessment of post- 
2128 treatment hypothyroidism, since it could be the result of the MMI rather than the RAI

2129 therapy.

2130 [Q2] Administration of RAI in the treatment of GD in children

\section{1 — RECOMMENDATION 69}

2132 If RAI therapy is chosen as treatment for GD in children, sufficient RAI should be

2133 administered in a single dose to render the patient hypothyroid. Strong

2134 recommendation, moderate-quality evidence.

2135 The goal of RAI therapy for GD is to induce hypothyroidism, rather than

2136 euthyroidism, as lower administered activities of RAI result in residual, partially

2137 irradiated thyroid tissue that is at increased risk for thyroid neoplasm development (351).

2138 Because of an increased risk of thyroid nodules and cancer associated with low-level

2139 thyroid irradiation in children (314,352-354), and poor remission rates with low-

2140 administered activities of RAI (88-90), it is important that RAI activities $>150 \mu \mathrm{Ci}$ (>

$21415.55 \mathrm{MBq} / \mathrm{g}$ ) rather than smaller activities of RAI be administered to achieve

2142 hypothyroidism (312). With large glands (50-80 g), RAI activities of ${ }^{131} \mathrm{I} 200-300 \mu \mathrm{Ci} / \mathrm{g}$

2143 (7.4-11.1 MBq/g) may be needed (349). The administered activity of RAI to patients with

2144 very large goiters is high, and there is a tendency to underestimate the size of the gland

2145 (and thereby administer insufficient RAI activities to these patients) (90). Therefore,

2146 surgery may be preferable to RAI in children with goiters larger than $80 \mathrm{~g}$.

2147 Physicians at some centers administer a fixed dose of about $15 \mathrm{mCi}$ RAI to all 2148 children (350), whereas others calculate the activity from estimation or direct 
2149 measurement of gland size and ${ }^{123}$ I uptake (349). To assess thyroid size, particularly in

2150 the setting of a large gland, ultrasonography is recommended (355). There are no data

2151 comparing outcomes of fixed versus calculated activities in children; in adults, similar

2152 outcomes have been reported with the two approaches (356). One potential advantage of

2153 calculated versus fixed dosing is that it may be possible to use lower administered

2154 activities of RAI, especially when uptake is high and the thyroid is small. Calculated

2155 dosing also will help assure that an adequate administered activity is given.

$2156 \quad$ When RAI activities $>150 \mu \mathrm{Ci} / \mathrm{g}(>5.55 \mathrm{MBq} / \mathrm{g})$ are administered,

2157 hypothyroidism rates are about $95 \%(88,339,349)$. While there are reports that

2158 hyperthyroidism can relapse in pediatric patients rendered hypothyroid with RAI, this is

2159 very infrequent.

2160 Technical remarks: RAI is excreted by saliva, urine, perspiration, tears, and

2161 stool. Significant radioactivity is retained within the thyroid for several days. It is

2162 therefore important that patients and families be informed of and adhere to local radiation

2163 safety recommendations following RAI therapy. After RAI therapy, $\mathrm{T}_{3}, \mathrm{~T}_{4}$, and/or free $\mathrm{T}_{4}$

2164 levels should be obtained every month. Because TSH levels may remain suppressed for

2165 several months after correction of the hyperthyroid state, TSH determinations may not be

2166 useful in this setting for assessing hypothyroidism. Hypothyroidism typically develops by

$21672-3$ months post-treatment $(333,349,350)$, at which time levothyroxine should be

2168 prescribed.

2169 [Q3] Side-effects of RAI therapy in children 
2171 hypothyroidism that is the goal of therapy. Fewer than $10 \%$ of children complain of mild

2172 tenderness over the thyroid in the first week after therapy; it can be treated effectively

2173 with acetaminophen or nonsteroidal antiinflammatory agents for 24-48 hours $(310,349)$.

2174 If there is residual thyroid tissue in young children after RAI treatment, there is a

2175 theoretical risk of development of thyroid cancer. Detractors of the use of RAI therapy in

2176 children point to the increased rates of thyroid cancer and thyroid nodules observed in

2177 young children exposed to radiation from nuclear fallout at Hiroshima or after the

2178 Chernobyl nuclear reactor explosion. However, these data do not apply directly when

2179 assessing risks of RAI therapy. The risk of thyroid neoplasia is greatest with exposure to

2180 low-level external radiation $(0.1-25 \mathrm{~Gy} ; \sim 0.09-30 \mu \mathrm{Ci} / \mathrm{g}$ or $3.33-1110 \mathrm{~Bq} / \mathrm{g})$

$2181(314,315,352,354,357)$, not with the higher administered activities used to treat GD. It is

2182 also important to note that iodine deficiency and exposure to radionuclides other than

2183 RAI may have contributed to the increased risk of thyroid cancer in young children after

2184 the Chernobyl reactor explosion (315). Notably, thyroid cancer rates were not increased

2185 among 3,000 children exposed to RAI from the Hanford nuclear reactor site in an iodine-

2186 replete region (358). Increased thyroid cancer rates also were not seen in 6,000 children

2187 who received RAI for the purpose of diagnostic scanning (359).

2188 There is no evidence to suggest that children or adults treated for GD with more

2189 than $150 \mu \mathrm{Ci} / \mathrm{g}(5.55 \mathrm{MBq} / \mathrm{g})$ of RAI have an increased risk of thyroid cancer directly

2190 attributable to RAI. While there are several studies of this issue in adults treated with RAI 
2191 for GD (see section [D2]), few studies have focused on populations exposed to RAI for

2192 the treatment of GD in childhood or adolescence.

In one study, an analysis was carried out of 602 individuals exposed to RAI below

219420 years of age in Swedish and U.S. populations (360). The average follow-up period

2195 was 10 years, and the mean administered activity of RAI to the thyroid was 88 Gy

2196 (approximately $80 \mu \mathrm{Ci} / \mathrm{g}$ or $2.96 \mathrm{MBq} / \mathrm{g}$ equivalent), an activity known to be associated

2197 with thyroid neoplasia and below that recommended for treatment of GD. Two cases of

2198 thyroid cancer were reported compared to 0.1 cases expected over that period of time.

2199 Effects on the development of nonthyroid cancers were not examined.

The pediatric study with the longest follow-up reported 36-year outcomes of 116 patients, treated with RAI between 1953 and 1973 (100). The patients ranged in age at treatment from 3 to 19 years. No patient developed thyroid cancer or leukemia. There was no increase in the rate of spontaneous abortion or in the number of congenital

2204 anomalies in offspring. It is important to note that sample size was small; thus, the 2205 statistical power was inadequate to address this issue fully.

2207 activities of RAI will result in more radiation exposure to a young child than to an 2208 adolescent or adult (361). At present, we do not have dosimetry information regarding 2209 RAI use in children with GD to assess total body exposure in children. Using phantom 2210 modeling, it has been estimated that at $0,1,5,10$, and 15 years of age, and adulthood, 2211 respective total body radiation activities are 11.1, 4.6, 2.4, 1.45, 0.90, and 0.85 rem (1 2212 rem $=0.1 \mathrm{~Sv}$ ) per mCi of RAI administered (361). Based on the Biological Effects of 
2213 Ionizing Radiation Committee VII analysis of acute, low-level radiation exposure (362),

2214 the theoretical lifetime attributable risk of all-cancer incidence and all-cancer mortality

2215 for a large population of treated children can be estimated (Table 9).

2216 To date, long-term studies of children treated with RAI for GD have not revealed

2217 an increased risk of nonthyroid malignancies. If a small risk exists, a sample size of more

2218 than 10,000 children who were treated at $<10$ years of age would be needed to identify

2219 the risk, likely exceeding the number of such treated children. Based on cancer risk

2220 projections from estimated whole-body, low-level radiation exposure as related to age, it

2221 is theoretically possible that there may be a low risk of malignancies in very young

2222 children treated with RAI. Thus, we recommended above that RAI therapy be avoided in

2223 very young children ( $<5$ years) and that RAI be considered in those children between 5

2224 and 10 years of age when the required activity for treatment is $<10 \mathrm{mCi}(<370 \mathrm{MBq})$. It is

2225 important to emphasize that these recommendations are based on theoretical concerns

2226 and further direct study of this issue is needed. The theoretical risks of RAI use must

2227 therefore be weighed against the known risks inherent in thyroidectomy or prolonged

2228 ATD use when choosing among the three different treatment options for GD in the

2229 pediatric age group.

The activity of RAI administered should be based on thyroid size and uptake, and

2231 not arbitrarily reduced because of age in young individuals. Attempts to minimize the

2232 RAI activity will result in undertreatment and the possible need for additional RAI

2233 therapy and radiation exposure. 
[R] If thyroidectomy is chosen as treatment for GD in children, how should it be

accomplished?

[R1] Preparation of children with GD for thyroidectomy

\section{- RECOMMENDATION 70} use of MMI. A potassium iodide containing preparation should be given in the

2241 immediate preoperative period. Strong recommendation, low-quality evidence.

2243 preferred treatment for GD in young children ( $<5$ years) when definitive therapy is

2244 required, and the surgery can be performed by a high-volume thyroid surgeon. In

2245 individuals with large thyroid glands $(>80 \mathrm{~g})$, the response to RAI may be poor $(88,90)$

2246 and surgery also may be preferable for these patients. When performed, near-total or total

2247 thyroidectomy is the recommended procedure (363).

2248 Technical remarks: MMI is typically given for 1-2 months in preparation for

2249 thyroidectomy. Potassium iodide (50 mg iodide/drop) can be given as 1-2 drops (i.e.,

$2250 \quad 0.05-0.1 \mathrm{~mL}$ ) three times daily for 10 days before surgery. SSKI can be mixed in juice or 2251 milk.

2253 If surgery is chosen as therapy for GD in children, total or near-total thyroidectomy 2254 should be performed. Strong recommendation, moderate-quality evidence. 
2256 Thyroidectomy in children should be performed by high-volume thyroid surgeons.

2257 Strong recommendation, moderately-quality evidence.

2258 Surgical complication rates are higher in children than in adults, with higher rates

2259 in younger than in older children $(316,318)$. Postoperatively, younger children also

2260 appear to be at higher risk for transient hypoparathyroidism than adolescents or adults

$2261 \quad(316,318)$.

Post-operative hypocalcemia requiring intravenous calcium infusions appears to

2263 occur more frequently than in in adults. Data from one center suggests that if calcitriol is

2264 started three days before surgery $(0.25$ or $0.5 \mathrm{mcg}$, bid $)$, the need for post-operative

2265 calcium infusions is markedly reduced, leading to reduction in the length of stay (318).

2266 The calcitriol is then weaned over the first two post-operative weeks (318).

In addition, complication rates are twofold higher when thyroidectomy is

2268 performed by pediatric or general surgeons who do not have extensive current experience

2269 in this procedure than when performed by high-volume thyroid surgeons (316). Further

2270 support for the notion that thyroidectomy for GD in children should be performed by

2271 experienced thyroid surgeons comes from reports of institutional experience showing low

2272 complication rates at high-volume centers $(318,364)$. In circumstances where local

2273 pediatric thyroid surgery expertise is not available, referral of a child with GD to a high-

2274 volume thyroid surgery center that also has pediatric experience is indicated, especially 
2275 for young children. A multidisciplinary health-care team that includes pediatric

2276 endocrinologists and experienced thyroid surgeons and anesthesiologists is optimal.

2278 [S] How should subclinical hyperthyroidism (SH) be managed?

The prevalence of $\mathrm{SH}$ in an adult population depends on age, gender, and iodine

2281 intake. In a representative sample of U.S. subjects without known thyroid disease, $0.7 \%$

2282 had suppressed TSH levels $(<0.1 \mathrm{mU} / \mathrm{L})$, and $1.8 \%$ had low TSH levels $(<0.4 \mathrm{mU} / \mathrm{L})$

2283 (365). Similar rates have been reported in studies from Europe, with higher levels in

2284 women and older subjects $(366,367)$. The differential diagnosis of an isolated low or

2285 suppressed TSH level includes exogenous thyroid hormone use, nonthyroidal illness,

2286 drug effects, and pituitary/hypothalamic disease, all of which need to be ruled out before

2287 the diagnosis of SH can be established in a patient with an isolated low or suppressed

2288 TSH level. In addition, mean serum TSH levels are lower in black non-Hispanic

2289 Americans, some of whom may have slightly low TSH levels without thyroid disease

2290 (365). Finally, some otherwise healthy older persons may have low serum TSH levels,

2291 low normal serum levels of free $\mathrm{T}_{4}$ and total $\mathrm{T}_{3}$, and no evidence of thyroid or pituitary

2292 disease, suggesting an altered set point of the pituitary-thyroid axis $(368,369)$.

The natural history of SH is variable (367,370-377), with annualized rates of $0.5-$

$22947 \%$ progression to overt hyperthyroidism and $5-12 \%$ reversion to normal TSH levels.

2295 In one study (372), 51.2\% of patients had spontaneously developed a normal TSH when 
2296 first checked at some time within 5 years (mean time to repeat TSH 13 months).

2297 Progression from SH to overt hyperthyroidism appears more likely if the TSH is

2298 suppressed $(<0.01 \mathrm{mU} / \mathrm{L})$, rather than low but detectable $(0.01-0.4 \mathrm{mU} / \mathrm{L})(375-377)$.

2299 Patients with GD rather than a TMNG as the cause of SH may be more likely to

2300 spontaneously remit $(367,378)$. In patients at high risk of complications from SH, TSH

2301 and free $\mathrm{T}_{4}$ should be repeated within 2-6 weeks. For all other patients, it is important to

2302 document that $\mathrm{SH}$ is a persistent problem by repeating the serum TSH at 3-6 months,

2303 prior to initiating therapy. In clinical series, TMNG is the most common cause of SH,

2304 especially in older persons $(367,376,377)$. The second most common cause of SH is GD,

2305 which is more prevalent in younger persons, and is also common in patients who

2306 previously received ATD therapy. Other unusual causes include solitary autonomously

2307 functioning nodules, and various forms of thyroiditis, the latter of which would be more

2308 strictly termed "subclinical thyrotoxicosis."

[S2] Clinical significance of SH

Since SH is a mild form of hyperthyroidism, it is not surprising that deleterious

2311 effects seen in overt hyperthyroidism might also occur in $\mathrm{SH}$. There have been a large

2312 number of recent studies elucidating these effects:

1. Overall mortality. A number of longitudinal studies have examined correlations

2314 between SH and overall mortality, with variable results. Some studies report

2315 increased overall mortality rates in SH subjects (374,379-383), especially older

2316 subjects, while others indicate no relation (384-387). Limitations of some of these studies include sample sizes, age ranges, length of follow-up, and diagnosis 
of SH by a single TSH measurement. A recent meta-analysis of individual-level data from 52,674 participants, pooled from 10 cohorts and providing greater power, concluded that SH confers a $24 \%$ increased risk of overall mortality (388).

2. Cardiovascular disease. A recent large study of 26,707 people followed for 12 years reported increased cardiovascular mortality with SH (389). Some other, smaller studies have reached similar conclusions (374,383), although other smaller studies have failed to find a correlation $(380,381,384,386)$. There have been two recent meta-analyses that examined this question, one of study-level data of 17 cohorts (390) and the other of individual-level data in 52,674 participants (388). Both analyses concluded that SH confers an increased risk of factors. However, the risk was greater in subjects with TSH levels $<0.1$ compared to those with TSH levels $0.1-0.4 \mathrm{mU} / \mathrm{L}$. non-fatal cardiovascular events in $\mathrm{SH}$, with similar increased risks $(383,388,390,391)$. The most recent data indicate that $\mathrm{SH}$ subjects appear to be at particular risk for the development of heart failure $(381,388,392)$, especially older subjects $(381,392)$ and those with lower TSH levels (392). Mechanistic correlates of these findings include increased left ventricular (LV) mass and impaired LV 2340 function in SH that improve with treatment (393-396). In addition, two studies 
have shown impaired glucose tolerance and decreased insulin sensitivity in $\mathrm{SH}$, suggesting this may contribute to increased cardiovascular risk $(397,398)$.

Arrhythmias are another concern in SH. Sawin et al first reported a 2.8fold increased risk of atrial fibrillation in SH subjects over age 60 years in 1994 (399), and subsequent studies have confirmed that the risk of arrhythmias, particularly atrial fibrillation, is increased in SH $(381,384,388,391,400,401)$. In the largest study to date $(586,460$ people followed for a median of 5.5 years), the highest relative risk for atrial fibrillation occurred in younger subjects, possibly because other causes predominate with age, and in subjects with lower TSH levels (401). However, absolute incidence rates of atrial fibrillation were much lower in younger subjects: for example, women under the age of 65 years had atrial fibrillation incidence rates of 2.3 events per 1,000 person-years (relative risk of 1.89 compared to age-matched euthyroid women), while women 65 years and older had incidence rates of 22.7 per 1,000 person-years (relative risk of 1.27 compared to age-matched euthyroid women). Similar trends were seen for men. A further population-based study found that $\mathrm{SH}$ increased the risk for stroke in subjects over age 50 years with a hazard ratio of 3.39 (402), although a recent meta-analysis of stroke risk in SH found insufficient number of events to draw definitive conclusions (403). Complementing these epidemiologic studies, investigations of smaller numbers of subjects with SH have revealed increased heart rate at rest and during exercise, decreased heart rate variability, and increased frequency of atrial and ventricular premature beats, which improve with treatment of SH $(393,394,404,405)$. 
Taken together, these data provide a strong argument for the treatment of SH in older subjects to avoid dysrhythmias and possible subsequent stroke. Whether younger patients should be treated for the same preventive indications is less clear. The most recent data provide evidence that relative risks of cardiovascular mortality and atrial fibrillation are elevated in younger, as well as older, patients with $\mathrm{SH}$. However, the absolute risks of these events are very low in younger patients, so the risk/benefit ratio of treating younger $\mathrm{SH}$ patients is not clear. Clinical judgement should be used in these cases, and treatment decisions individualized.

3. Osteoporosis and fractures. Most studies of endogenous SH show decreased bone mineral density in post-menopausal women, but not in men or premenopausal women (406). However, it is not clear that this translates to increased fracture risk. A number of population-based studies have reported that certain groups of subjects with SH have increased fracture rates, including all adults (407), postmenopausal women (408), men (409), or subjects who progress to overt hyperthyroidism over time (391). The most recent and by far the largest individual study to date $(231,355$ people) reported a hazard rate for all major osteoporotic fractures combined (hip, humerus, forearm, spine) of 1.13 (confidence intervals 1.014-1.26). Risk increased with duration of $\mathrm{SH}$, such that after a median follow-up of 7.5 years, $13.5 \%$ of subjects with a low TSH level had experienced at least one major osteoporotic fracture, compared to $6.9 \%$ of subjects with a normal TSH level (407). Other studies have not found increased fracture rates in SH subjects (410-412). A recent participant-level meta-analysis 
of 13 cohorts (70,298 participants, median follow-up of 12.1 years) concluded that $\mathrm{SH}$ subjects had significantly elevated hazard ratios of 1.36 for hip fractures (6 vs. 4.9 fractures per 1,000 person-years) and 1.28 for any fractures (14.4 vs 11.2 fractures per 1,000 person-years) (413). Risks were further increased if TSH levels were $<0.1$, compared to $0.1-0.44 \mathrm{mU} / \mathrm{L}$, and if SH was due to endogenous etiologies, rather than thyroid hormone administration. Risks did not differ when stratified by age, although absolute fracture rates were lower in younger subjects. There are smaller, nonrandomized trials that have shown improvement in bone mineral density with therapy of $\mathrm{SH}$ with antithyroid drugs or radioactive iodine (414-417).

4. Mood and cognition. A large body of literature has investigated possible correlations between SH and cognitive decline (reviewed in (418), more recent studies $(419,420))$. Approximately equal numbers of studies report significant associations between $\mathrm{SH}$ and measures of cognitive decline and the development of dementia, vs. no associations. Therefore, at this time, no conclusions regarding this issue can be reached. There appears to be no correlation between SH and depression (421-423).

5. Physical functioning. Four studies have investigated whether $\mathrm{SH}$ is associated with self-reported functional capacity or objective measures of physical functioning (420,423-425). Three could find no correlation, while the fourth found a correlation between $\mathrm{SH}$ and lower physical performance in men only (425). Another uncontrolled study showed an increase in muscle mass and 

thyroidectomy (426).

$2411 \quad[\mathrm{~S} 3]$ When to treat $\mathrm{SH}$

\section{2 - RECOMMENDATION 73}

2413 When TSH is persistently $<0.1 \mathrm{mU} / \mathrm{L}$, treatment of $\mathrm{SH}$ is recommended in all

2414 individuals $\geq 65$ years of age; in patients with cardiac risk factors, heart disease or

2415 osteoporosis; in postmenopausal women who are not on estrogens or bisphosphonates;

2416 and in individuals with hyperthyroid symptoms. Strong recommendation, moderate-

2417 quality evidence.

\section{8 - RECOMMENDATION 74}

2419 When TSH is persistently $<0.1 \mathrm{mU} / \mathrm{L}$, treatment of SH should be considered in

2420 asymptomatic individuals $<65$ years of age without the risk factors listed in

2421 recommendation 73 . Weak recommendation, moderate-quality evidence.

2423 have been performed, especially for clinically important endpoints such as cardiovascular

2424 events, atrial fibrillation, and fractures. Additionally, none of these studies included a

2425 control arm. Thus the evidence rests only with small uncontrolled studies that have

2426 shown improvements in cardiac structure and function, heart rate and the frequency of

2427 premature atrial and ventricular beats, bone mineral density, and muscle strength (393-

$2428396,405,414-417,426)$. In 2004, a panel of experts determined that the evidence for 
2429 benefit was sufficient to warrant therapy of SH in older individuals whose serum TSH

2430 level was $<0.1 \mathrm{mU} / \mathrm{L}$ (427). This was based primarily on the studies showing an

2431 increased rate of atrial fibrillation and altered skeletal health with a suppressed level of

2432 TSH described above. Emerging epidemiologic data since then on risks for overall and

2433 cardiovascular-specific mortality, summarized above, have strengthened this argument,

2434 even in the absence of interventional data. The European Thyroid Association recently

2435 reviewed these data and published guidelines for the treatment of subclinical

2436 hyperthyroidism which are largely concordant with recommendations presented here

2437 (428).

2438 There are insufficient data for or against treatment of SH in younger persons or

2439 premenopausal women with SH and serum TSH $<0.1 \mathrm{mU} / \mathrm{L}$. One uncontrolled study of

2440 middle-aged patients showed an improvement in hyperthyroid symptoms with therapy

2441 (393). Although this study did not include younger individuals, the task force elected to

2442 recommend treatment of SH patients younger than 65 years of age with persistent TSH

$2443<0.1 \mathrm{mU} / \mathrm{L}$ and hyperthyroid symptoms. In the absence of symptoms or risk factors,

2444 treatment decisions must be individualized.

2445 Technical remarks: A TSH level of $<0.1 \mathrm{mU} / \mathrm{L}$ on repeated measurement over a

2446 3-6-month period is considered to be persistent, effectively ruling out transient thyroiditis

2447 as a cause. The thyroid disorder underlying SH should be diagnosed, and is most

2448 commonly TMNG, GD, or TA.

2449 - RECOMMENDATION 75 
2450 When TSH is persistently below the lower limit of normal but $\geq 0.1 \mathrm{mU} / \mathrm{L}$, treatment

2451 of SH should be considered in individuals $\geq 65$ years of age and in patients with

2452 cardiac disease, osteoporosis, or symptoms of hyperthyroidism. Weak

2453 recommendation, moderate-quality evidence.

2454 - RECOMMENDATION 76

2455 When TSH is persistently below the lower limit of normal but $\geq 0.1 \mathrm{mU} / \mathrm{L}$,

2456 asymptomatic patients under age 65 without cardiac disease or osteoporosis can be

2457 observed without further investigation of the etiology of the subnormal TSH or

2458 treatment. Weak recommendation, low-quality evidence.

A number of the epidemiologic studies listed above performed analyses for $\mathrm{SH}$

2460 subjects with low but detectable TSH levels (generally 0.1 to $0.4 \mathrm{mU} / \mathrm{L}$ ). Some of these

2461 studies reported increased risks of overall mortality in older subjects $(380,429)$,

2462 cardiovascular events (391), heart failure (381), and atrial fibrillation in all subjects (401)

2463 or in older subjects (384), and vertebral fractures in older women (408). However, there

2464 are no interventional data for or against treatment of individuals with serum TSH levels

2465 between 0.1 and the lower limit of the reference range. Therefore, treatment decisions

2466 must be individualized, based on the limited epidemiologic evidence and patient risk

2467 factors. The task force felt that the limited data are stronger for older subjects, and

2468 therefore treatment should be considered for older subjects, but is not recommended for

2469 subjects $<65$ years of age. However, younger subjects should be monitored at regular 6-

247012 month intervals, and treatment should be considered if the TSH persistently decreases 
2471 to $<0.1 \mathrm{mU} / \mathrm{L}$. In patients with symptoms of hyperthyroidism, a trial of beta-adrenergic

2472 blockers may be useful to determine whether symptomatic therapy might suffice.

2474 measurement over a 3-6-month period is considered persistent, effectively ruling out

2475 transient thyroiditis as a cause. The thyroid disorder underlying SH with TSH persistently

2476 within this range should be diagnosed before considering treatment to avoid treating

2477 patients with transient, functional disorders related to acute illness, drugs, and other

2478 causes of low TSH. A summary of factors to consider when deciding whether or not to

2479 treat a patient with SH is provided (Table 10).

$2480 \quad[\mathrm{~S} 4]$ How to treat SH

\section{- RECOMMENDATION 77}

2482 If $\mathrm{SH}$ is to be treated, the treatment should be based on the etiology of the thyroid 2483 dysfunction and follow the same principles as outlined for the treatment of overt 2484 hyperthyroidism. Strong recommendation, low-quality evidence.

2485 The treatment of SH is similar to the treatment of overt hyperthyroidism.

- RAI is appropriate for most patients, especially in older patients when TMNG is a frequent cause of $\mathrm{SH}$. There are no data to inform whether elderly patients with SH would benefit from pretreatment with ATDs to normalize thyroid function before RAI therapy. Given the low risk of exacerbation (71), the risks of ATD therapy may outweigh any potential small benefit. 
- A course of ATD therapy is a reasonable alternative to RAI in patients with GD and $\mathrm{SH}$, especially in younger patients, since remission rates are highest in persons with mild disease (109).

- Some patients with SH due to GD may remit spontaneously without therapy (375-377), so that continued observation without therapy is reasonable for adrenergic blockade may be sufficient to control the cardiovascular-related morbidity from $\mathrm{SH}$, especially that of atrial fibrillation (430).

2503 spontaneously and may be followed without therapy with frequent (every 3-6 months)

2504 monitoring of thyroid function. In select patients with SH due to TMNG who have

2505 compressive symptoms, or in whom there is concern for malignancy, surgery is also an 2506 option.

[S5] End points to be assessed to determine effective therapy of $\mathrm{SH}$ The goal of therapy for SH is to render the patient euthyroid with a normal TSH.

2509 Since the rationale for therapy of SH is to a large degree preventive, there are few end 2510 points that can be used to document that therapy has been successful. Based on the 2511 original indication for treatment, it is reasonable to follow hyperthyroid symptoms or 
2512 bone density $(393,414-416)$; otherwise, the major end point is a TSH level within the age-

2513 adjusted reference range.

\section{[T] How should hyperthyroidism in pregnancy be managed?}

2516 Normal pregnancy leads to changes in thyroid physiology that are reflected by

2517 altered thyroid function testing. In early pregnancy, these changes can mimic biochemical

2518 hyperthyroidism that does not require therapy (431). Hyperthyroidism due to GD occurs

2519 in $0.5-1.0 \%$ of women in the reproductive age range (432), and $0.1-0.2 \%$ of them are

2520 treated with ATD during pregnancy $(433,434)$. Both the thyrotoxicosis and therapy of the

2521 disease may seriously complicate the course and outcome of pregnancy. In these

2522 guidelines, we will address only the most common issues related to hyperthyroidism in

2523 pregnancy, pending full guidelines on thyroid disease and pregnancy that are currently

2524 being updated by the ATA.

2525 [T1] Diagnosis of hyperthyroidism in pregnancy

2526 - RECOMMENDATION 78

2527 The diagnosis of hyperthyroidism in pregnancy should be made using serum TSH

2528 values, and either total $\mathrm{T}_{4}$ and $\mathrm{T}_{3}$ with total $\mathrm{T}_{4}$ and $\mathrm{T}_{3}$ reference ranges increasing to

$2529 \quad 1.5$ times above the nonpregnant range by the $2^{\text {nd }}$ and $3^{\text {rd }}$ trimester or free $\mathrm{T}_{4}$ and total

$2530 \mathrm{~T}_{3}$ estimations with trimester-specific normal reference ranges. Strong

2531 recommendation, low-quality evidence. 
2533 majority of patients, the disease is caused by a primary thyroid abnormality, and the 2534 principal finding will be a suppressed serum $\mathrm{TSH}$, with serum free $\mathrm{T}_{4}$ (or total $\mathrm{T}_{4}$ ) and/or $2535 \mathrm{~T}_{3}$ levels above the reference range (overt hyperthyroidism), or within the reference range $2536(\mathrm{SH})$. A key point is that reference ranges for thyroid function tests are different during 2537 different stages of pregnancy, and these changes may be assay-dependent. An understanding of pregnancy-related variations in thyroid function tests is 2539 important in making the diagnosis of hyperthyroidism in pregnancy. Serum TSH levels 2540 may be below the non-pregnant reference range in the first half of a normal-term 2541 pregnancy $(435,436)$, and especially so in gestational weeks $9-13$, where a subset of 2542 pregnant women may develop suppressed serum TSH (437-439). The decrease in TSH in 2543 early pregnancy is the result of stimulation of the normal thyroid by high levels of serum 2544 human chorionic gonadotropin (hCG) (440), and occasionally the biochemical findings 2545 developing may correspond to overt thyrotoxicosis (gestational hyperthyroidism 2546 discussed below). However, low serum TSH levels with normal free $\mathrm{T}_{4}$ (or total $\mathrm{T}_{4}$ ) in 2547 early pregnancy do not indicate disease in need of therapy. During the second half of 2548 pregnancy, the lower limit of normal for TSH in the non-pregnant population can be used 2549 (441).

Free $\mathrm{T}_{4}$ and $\mathrm{T}_{3}$ measured in an equilibrium dialysate or an ultrafiltrate of serum 2551 around week 10 of pregnancy may be slightly higher (5-10\%) than non-pregnancy 2552 values, corresponding to the period of high serum hCG and low serum TSH. From 
2553 normal or slightly elevated levels, a gradual decrease occurs during pregnancy, and late

2554 third trimester reference values are 10-30\% below non-pregnancy values (442).

Serum total $\mathrm{T}_{4}$ and $\mathrm{T}_{3}$ increase in parallel in early pregnancy, primarily due to

2556 increases in TBG. In one longitudinal study, the increase in $\mathrm{T}_{4}$ and $\mathrm{T}_{3}$ reference ranges

2557 were observed to occur at a rate of $5 \%$ of non-pregnant values per week over the 10 week

2558 period of gestation weeks 7-16 (443). After this 50\% increase, total $\mathrm{T}_{4}$ and $\mathrm{T}_{3}$ values

2559 remain stable with reference range limits 1.5 times above non-pregnancy ranges over the

2560 remaining weeks of pregnancy $(442,443)$. Total $\mathrm{T}_{4}$ and $\mathrm{T}_{3}$ values may be combined with

2561 a $T_{3}$ uptake test or measurements of TBG to adjust for pregnancy-associated variations in

2562 TBG. Such "free $\mathrm{T}_{4}$ index" or "TBG adjusted $\mathrm{T}_{4}$ " values may be useful for diagnosing

2563 hyperthyroidism in pregnancy, however, trimester-specific normal reference ranges

2564 should be established for each individual test and assay used. In the absence of these,

2565 consideration should be given to utilizing total $\mathrm{T}_{4}$ and $\mathrm{T}_{3}$ levels and multiply the non-

2566 pregnancy reference range by 1.5 after week 16, as discussed above.

\section{Excluding patients with TSH suppression or gestational thyrotoxicosis}

2568 during the first trimester, GD is the most common cause of hyperthyroidism during

2569 pregnancy $(431,444)$; nodular thyroid disease is less common. Hyperthyroidism caused

2570 by a hCG-producing molar pregnancy or a choriocarcinoma presents with a diffuse

2571 hyperactive thyroid similar to GD, but without eye signs and without TRAb being

2572 detectable in serum. In these patients, serum hCG will be higher than expected, and the 2573 cause can be identified by obstetrical investigation. 
2575 and free $T_{3}$ has been questioned for more than 25 years (445), but these estimates are

2576 currently widely used because of their suitability for large scale automatic analyses

2577 within short time periods. In many clinics, they are the standard of measurement in

2578 pregnancy. Because pregnancy may influence results of these assays from different

2579 manufacturers in different ways, and in some assays give spuriously low results (446),

2580 method-specific reference ranges for each trimester of pregnancy should be used and

2581 provided by the manufacturer $(447,448)$. If trimester specific references for free $\mathrm{T}_{4}$ (and

2582 free $\mathrm{T}_{3}$ ) are not provided, and total $\mathrm{T}_{4}$ (and $\mathrm{T}_{3}$ ) assays are not locally available, samples

2583 for thyroid function testing in pregnancy should be send to a reference laboratory.

2584 [T2] Management of hyperthyroidism in pregnancy

2585 Table 11 provides a summary of the recommendations concerning management of

2586 GD during pregnancy.

\section{- RECOMMENDATION 79}

2588 Transient hCG-mediated TSH suppression in early pregnancy should not be treated 2589 with antithyroid drug therapy. Strong recommendation, low-quality evidence.

2590 Once the diagnosis of hyperthyroidism is made in a pregnant woman, attention

2591 should focus on determining the etiology and whether it warrants treatment. Clinical

2592 features that indicate the presence of hyperthyroidism include failure to gain weight, heat

2593 intolerance, excessive sweating, and tachycardia, beyond that normally associated with 2594 pregnancy. 
The two most common types of biochemical hyperthyroidism that occur during

2596 pregnancy are gestational hyperthyroidism (e.g., hCG-mediated transient TSH

2597 suppression) and GD. Gestational hyperthyroidism is a generally asymptomatic, mild and

2598 self-limiting biochemical hyperthyroidism that may be observed in the first trimester of

2599 normal pregnancy. The disorder lacks the characteristics of Graves' disease (431), and is

2600 presumably caused by the high serum hCG of early pregnancy (440). It is not associated

2601 with adverse pregnancy outcomes (449). More severe degrees of gestational

2602 hyperthyroidism are associated with hyperemesis; affected women may develop

2603 biochemically overt hyperthyroidism and clinical symptoms and signs of

2604 hyperthyroidism. Complicated cases of gestational hyperthyroidism should be referred to

2605 medical centers with expertise in treating these patients.

Technical remarks: There is no evidence that treatment of gestational

2607 hyperthyroidism with ATDs is beneficial and use of ATD in early pregnancy has been

2608 associated with an increase in risk of birth defects. In these patients, physical examination

2609 and repeat thyroid function tests at intervals of 3-4 weeks is recommended. In the case of

2610 very symptomatic disease, a trial of beta blocker therapy (propranolol or metroprolol, but

2611 not atenolol $(450,451))$ for this transient disorder may be considered.

2612 - RECOMMENDATION 80

2613 ATD therapy should be used for overt hyperthyroidism due to GD during pregnancy.

2614 PTU should be used when ATD therapy is given during the first trimester. MMI

2615 should be used when ATD therapy is started after the first trimester. Strong

2616 recommendation, low-quality evidence. 
2618 pregnancy (452-454), and patients with this disorder should be treated at centers with

2619 specific expertise in this area. GD as the cause of hyperthyroidism in pregnancy may be

2620 diagnosed from typical clinical findings, including the presence of GO and/or serum

2621 TRAb in a hyperthyroid patient. Approximately 5\% of patients with newly diagnosed

2622 Graves' hyperthyroidism are TRAb negative in older assays $(47,455)$, and $3 \%$ are

2623 negative in third generation assays (57), especially those with milder disease.

A small increase in incidence of GD was found in early pregnancy in one study

2625 (456), and this fits the clinical observation that existing GD may occasionally worsen in

2626 early pregnancy (457). On the other hand, the incidence of GD drops dramatically in late

2627 pregnancy (456), which is consistent with the notion that thyroid autoimmunity improves

2628 in the second half of pregnancy (458).

Women who were treated with ATDs for GD and considered in remission after

2630 such previous therapy, and become pregnant, have a small risk of recurrence and should

2631 have their thyroid function tested in early pregnancy. In contrast, the risk of relapse (as

2632 well as the risk of thyrotoxicosis from postpartum destructive thyroiditis) during the

2633 postpartum period is relatively high (459), and it remains elevated for more than one year

2634 (456).

ATDs have much the same effect on thyroid function in pregnant as in non-

2636 pregnant women. Both ATDs and TRAb pass the placenta and can affect the fetal

2637 thyroid. On the other hand, $\mathrm{T}_{4}$ and $\mathrm{T}_{3}$ cross the placenta only in limited amounts, because

2638 of degradation by high deiodinase type 3 activities in the placenta (460). 
PTU generally has been preferred in pregnancy because of concerns about well-

2640 documented teratogenicity associated with MMI, first described in 1972 (461). Defects

2641 that may be observed in 2-4\% of exposed children $(462,463)$ have included aplasia cutis,

2642 choanal atresia, esophageal and other types of gut atresias, abdominal wall abnormalities

2643 including omphalocale, eye, heart, and urinary tract malformations. Moreover, typical

2644 facial features of MMI-exposed children have been described in case reports (464). In a

2645 US study, $31 \%$ of women who had received MMI around the time of conception had

2646 elective termination of pregnancy versus $9 \%$ of those who received PTU, and it was

2647 hypothesized that fear of MMI associated birth defects had led to the decision to

2648 terminate pregnancy (465).

2649 Recently, an increase in the rate of birth defects $(2.3 \%$ above the background 2650 rate) was also observed after PTU exposure in early pregnancy (463), but these defects 2651 tended to be less severe than with MMI and included preauricular sinuses and cysts and 2652 urinary tract abnormalities (466). In a large group of children selected because they had 2653 major birth defects and had been exposed to some type of medication in early pregnancy, 2654 children exposed to PTU had a significantly higher frequency of situs inversus and 2655 cardiac outflow abnormalities than children exposed to other drugs (467), but these types 2656 of defects have not been observed in excess in studies comparing PTU exposed children 2657 with non-selected control children. Similar to other teratogenic drugs (468) the period of 2658 highest risk for birth defects from ATDs is gestational weeks 6-10 (469). Concerns about rare but potentially fatal PTU-related hepatotoxicity have led The 2660 U.S. Food and Drug Administration to recommended that PTU be reserved for patients 
2661 who are in their first trimester of pregnancy, or who are allergic to or intolerant of MMI $2662(157,470)$

MMI and PTU both appear in breast milk in only small concentrations and studies

2664 of breast-fed infants of mothers taking ATDs have demonstrated normal thyroid function

2665 and subsequent normal intellectual development (109). However, because of the potential

2666 for hepatic necrosis in either mother or child from maternal PTU use, MMI is the

2667 preferred ATD in nursing mothers.

2668 As discussed in other sections of these guidelines, small doses of beta-adrenergic

2669 blocking agents are in general useful to reduce pulse rate and the hyperadrenergic

2670 symptoms of thyrotoxicosis during the time period from start of ATD therapy until the

2671 patient has become euthyroid. These agents have been studied extensively when used for

2672 treating hypertension in pregnancy, and no major side-effects have been detected,

2673 although fetal growth restriction has been associated with the prolonged use of especially

2674 atenolol $(431,471)$. Therapy with propranolol (e.g. 10-20 mg every eight hour) or

2675 metopolol (e.g. $100 \mathrm{mg}$ once daily) are useful and can be considered safe for short

2676 periods of time to relieve symptoms in pregnant women suffering from thyrotoxicosis.

2677 - RECOMMENDATION 81

2678 In women who develop hyperthyroidism during their reproductive age range, the

2679 possibility and timing of future pregnancy should be discussed. Because of the risks of

2680 the hyperthyroid state on pregnancy and fetal outcome, we suggest that women should 
2681 postpone pregnancy until they have become euthyroid with therapy. Strong

2682 recommendation, low-quality evidence.

Both maternal thyroid dysfunction and therapy of the hyperthyroidism may have

2684 negative effects on the pregnancy outcome. These factors should all be considered when

2685 determining the choice of therapy for the patient who is currently pregnant, or in the

2686 future may become pregnant.

A single set of thyroid function tests within the reference range may not guarantee

2688 euthyroidism for more than a short period during the early phase of hyperthyroidism

2689 therapy. Two sets of tests within the reference range, taken with an interval of at least one

2690 month and without a change of therapy is preferable to indicate euthyroidism.

\section{- RECOMMENDATION 82}

2692 We suggest that women with hyperthyroidism caused by GD that require high doses of 2693 ATDs to achieve euthyroidism should be considered for definitive therapy before they 2694 become pregnant. Weak recommendation, low-quality evidence.

2696 permanently hypothyroid with the possibility of a stable euthyroid state on thyroid

2697 hormone replacement therapy, as discussed in these guidelines. Thyroidectomy is often

2698 followed by a decrease or disappearance of TRAb from circulation, whereas RAI is

2699 often followed by a transient increase in TRAb. This is a potential argument in favor of

2700 surgical thyroidectomy in women with high TRAb titers that may become pregnant

2701 within the years to come, especially those planning therapy within the next year (172). 
2702 However, the importance of this difference in autoimmune activity for pregnancy

2703 outcome has not been studied, and it should be weighed against the other benefits and

2704 harms of surgery and RAI therapy.

To predict reduction in TRAb after surgical thyroidectomy, a recent retrospective

2706 Japanese study of 45 (41 female) patients with high TRAb (median 64 IU/L, range 5.6-

2707400 , normal for assay < 1.9 IU/L) may be useful. Patients were followed for 12 months.

2708 Smoking and the presence of orbitopathy predicted slow disappearance of TRAb (half-

2709 life 162 days, or 357 days if both factors were present), whereas TRAb levels in serum

2710 decreased with a half-life of 94 days in the remaining patients (472).

2711 Medical tradition and experience with different types of therapy for GD varies

2712 between countries and clinics, and the risk of relapse of hyperthyroidism after ATD

2713 withdrawal may differ considerably, depending on iodine intake, and other factors that

2714 are only partly understood (473). Thus, advice given to women with GD on therapy in

2715 relation to a possible future pregnancy may differ. However, irrespective of such

2716 differences, the physician providing care to a young woman with newly diagnosed GD,

2717 should include discussion and guidance on GD and pregnancy. The severely hyperthyroid

2718 patient may not be in a position to fully comprehend many simultaneous messages, and a

2719 more detailed discussion may be appropriate when the patient has become euthyroid.

2720 - RECOMMENDATION 83

2721 Women with hyperthyroidism caused by GD who are well controlled on MMI and 2722 desire pregnancy have several options: 
a) Patients could consider definitive therapy before they become pregnant.

b) Patients could switch to PTU before trying to conceive.

c) Patients could switch to PTU as soon as pregnancy is diagnosed.

d) Appropriately selected patients could withdraw from ATD therapy as soon as

2727 pregnancy is diagnosed. If ATD therapy is withdrawn, thyroid function should be

2728 assessed weekly throughout the first trimester, then monthly. Weak

2729 recommendation, low-quality evidence.

The evidence is insufficient to give universal guidance on how to choose among

2731 these options, and therefore the potential risks and benefits of each option should be

2732 discussed with the patient, and patient values and preferences should be taken into 2733 account.

Each option is presented in depth in the following technical remarks:

\section{A. Definitive therapy before becoming pregnant.}

This strategy is discussed in Recommendation 82. It has the advantage of

2737 allowing the patient to become pregnant free of worry from the adverse fetal effects of

2738 ATDs. The disadvantage is that the patient will require levothyroxine therapy while

2739 pregnant and lifelong, and will be exposed to either the potential complications of RAI,

2740 including worsening or induction of Graves' orbitopathy, or the potential for undesirable 2741 surgical outcomes. 
B. Switching from MMI to PTU before pregnancy.

2745 early pregnancy exposure to MMI in women where pregnancy is not recognized within

2746 the first few weeks after conception. MMI associated birth defects occur in 2-4 \% of

2747 children exposed in early pregnancy, and abnormalities may be severe. PTU associated

2748 birth defects are less well documented. They may occur in 2-3\% of children but they

2749 seem to be mostly less severe. PTU is associated with liver failure with an estimated

$27501: 10,000$ risk of severe liver failure in adult patients (136). Thus, mothers must balance

2751 the risk of PTU to themselves vs. the risk to the child. Switching to PTU before

2752 conception may be preferred in younger women with regular menses who are expected

2753 to be able to conceive within 1 to 3 months. In a German prospective study of 340 such

2754 women, $68 \%$ became pregnant within 3 months (474).

2756 they hope to become pregnant soon. There are not sufficient data to recommend for or

2757 against starting therapy with PTU and thus bypass a phase of MMI therapy in such

2758 patients.

2759 C. Switching from MMI to PTU after conception.

Alternatively, the patient may continue MMI therapy but be prepared to detect

2761 pregnancy very early and modify therapy immediately as recommended below.

2762 Switching to PTU as soon as pregnancy is diagnosed may be preferred in older women 
2763 and women who have conditions that may be associated with delayed conception. This

2764 strategy may prevent prolonged use of PTU prior to conception but has the risk of fetal

2765 exposure to MMI if the diagnosis of pregnancy is delayed.

2766 D. Withdrawing ATD treatment after conception.

2767 Women with a stable euthyroid state on 5-10 mg MMI per day achieved within a

2768 few months, and a falling TRAb level are likely candidates to withdraw from ATD

2769 therapy in early pregnancy.

No study has directly addressed the risk of relapse of hyperthyroidism after ATD

2771 withdrawal in early pregnancy, and evidence comes from controlled or cohort studies of

2772 non-pregnant patients who had been treated with ATD for varying periods before drug

2773 withdrawal. Based on the latter studies, the risk of relapse of hyperthyroidism within a

2774 two month interval after ATD withdrawal in TRAb negative, non-smoking patients who

2775 have already been treated for $12-24$ months is $<10 \%(167,475)$.

2776 On the other hand, the risk of early relapse is very high in patients who have

2777 received ATD for less than six months, and/or still have indicators of high disease

2778 activity such as low serum TSH, high TRAb level, signs of active GO, or need of MMI

2779 dose in excess of 5-10 mg per day to remain euthyroid (473).

If ATD withdrawal is followed by a relapse of hyperthyroidism, this will often

2781 develop gradually over some weeks, but exact information on such time course in early

2782 pregnancy is not available. This is the reason for the recommendation of frequent thyroid 
2783 function testing during the remaining $1^{\text {st }}$ trimester of pregnancy, until more data on safety 2784 becomes available.

A subset of women with GD will experience relapse of hyperthyroidism in 2786 pregnancy if ATD therapy is withdrawn according to recommendation 81. Frequent 2787 testing of thyroid function will allow early detection of such relapse and initiation of 2788 therapy with PTU (or MMI if relapse occurs in the second trimester) to keep the 2789 mother euthyroid. The risk to the mother from such hyperthyroidism is considered 2790 negligible.

Considering the fetus, two recent studies performed in Japan suggest that such

2792 transient and mild maternal hyperthyroidism will not increase the risk of malformations.

2793 One study observed a significantly lower risk of birth defects in mothers who had been 2794 shifted from MMI to iodine therapy in early pregnancy, even if part of the mothers in the 2795 iodine group had developed biochemical hyperthyroidism and needed retreatment with 2796 ATD (476). In another study from the same institution, the presence of a major birth 2797 defect was associated with the use of MMI in early pregnancy, but not with maternal 2798 thyroid dysfunction (462).

A more pertinent risk may be fetal loss caused by maternal hyperthyroidism in 2800 pregnancy $(477,478)$. However, the risk from a brief period of mild maternal thyroid 2801 hyperfunction in early pregnancy may be low or absent. In a large cohort of pregnant 2802 women from the USA, low or suppressed serum TSH in early pregnancy (presumably 2803 mostly caused by early pregnancy high hCG levels) was not associated with adverse 2804 pregnancy outcomes (449). In the recent retrospective Japanese study of women with GD 
2805 either treated with MMI in early pregnancy or shifted from MMI to iodine therapy in

2806 early pregnancy, there was no increase in fetal loss in the iodine group despite more cases

2807 of maternal hyperthyroidism in this group (476).

\section{- RECOMMENDATION 84}

2809 We suggest that women who are treated with ATD and who may potentially become

2810 pregnant should be instructed to perform a pregnancy test within the first days after a

2811 missed or unusually light menstrual period. Weak recommendation, low-quality

2812 evidence.

2813 The period of major risk of birth defects caused by intake of medication in

2814 pregnancy is gestational weeks 6-10 (468), and a study of time of exposure to ATD and

2815 risk of defects suggests this is also the major period of teratogenic effects of ATD (469).

2816 Thus, withdrawal of ATD therapy before week five of pregnancy may theoretically

2817 prevent birth defects caused by ATD exposure.

The week of pregnancy is calculated starting from the first day of the last normal

2819 menstrual period, with conception taking place about two weeks after this. The first real

2820 sign of pregnancy appears two weeks later, and it is a missed or unusually light menstrual

2821 period. By this time, blood and urine concentrations of hCG have started to rise and

2822 generally available pregnancy tests based on detection of hCG in urine normally become

2823 positive early in gestational week five. Very early testing for pregnancy to allow

2824 medication withdrawal before the major period of teratogenecity is recommended for

2825 other types of drugs that may be teratogenic (479). 
2827 We suggest that a woman who tests positive for pregnancy according to

2828 recommendation 84 contact the physician responsible for the ATD therapy within 24

2829 hours to discuss future treatment options. Weak recommendation, low-quality

$2830 \quad$ evidence.

The time window that will allow medication withdrawal or change in early

2832 pregnancy to prevent birth defects is narrow $(468,469)$, probably confined to gestational

2833 week 5. Thus, pregnancy should be detected early and action has to be taken

2834 immediately.

\section{- RECOMMENDATION 86}

2836 We suggest that the physician contacted according to recommendation 85 evaluate

2837 whether ATD withdrawal in the first trimester of pregnancy is likely to cause relapse

2838 of hyperthyroidism or not. Evaluation should be based on patient records, especially

2839 the severity of GD at time of diagnosis and current disease activity, duration of ATD

2840 therapy, current ATD dose requirement, and results of recent thyroid function and

2841 TRAb testing. If risk of relapse is considered low, therapy can be withdrawn, and

2842 followed by weekly thyroid function testing during the $1^{\text {st }}$ trimester. Weak

2843 recommendation, low-quality evidence.

In the majority of patients with GD, ATD therapy is followed by a gradual

2845 remission of disease with a possibility of disappearance of TRAb from circulation (172).

2846 When patients have been treated with ATD for 12-18 months a rapid relapse of 
2847 hyperthyroidism after ATD withdrawal becomes less likely (119), even if the frequency

2848 of relapse may be in the order of $50 \%$ within one year. The risk of relapse after ATD

2849 withdrawal varies considerably among individual patients and it depends on a variety of

2850 factors (473), as discussed in detail above.

\section{1 - RECOMMENDATION 87}

2852 We suggest that women in early pregnancy who have a high risk of recurrent or

2853 worsening hyperthyroidism if ATD is withdrawn be shifted from MMI to PTU

2854 immediately after diagnosing pregnancy. Weak recommendation, low-quality

2855 evidence.

Even if birth defects may occur after both MMI and PTU exposure in early

2857 pregnancy (463), defects after MMI exposure are better documented. The reason for this

2858 seems to be that MMI associated defects are more severe, whereas PTU associated

2859 defects tend to be less severe and may not be diagnosed immediately after birth (466).

2860 Both MMI and PTU are effective therapies of hyperthyroidism in the majority of

2861 patients and the major effect of both drugs is to interact with thyroid peroxidase catalyzed

2862 thyroid hormone production (109). Apart from the differences in side-effects discussed

2863 above, it is important to consider differences in potency per mg drug and in duration of

2864 effect.

A dosage ratio of MMI to PTU of 1:20 is recommended when changing from one

2866 drug to another $(115,319,480)$, although only two studies have examined this dosage ratio

2867 directly $(115,319)$. Moreover, the difference in duration of effect should be taken into 
account. For example, $15 \mathrm{mg}$ of MMI would be roughly equivalent to $300 \mathrm{mg}$ of PTU,

2869 but because the half-life of PTU is considerably shorter than that of MMI, the dose of

2870 PTU should be split over the day $(481,482)$, e.g. MMI 15 mg once daily may be

2871 substituted with PTU $100 \mathrm{mg}$ three times a day (319).

\section{- RECOMMENDATION 88}

2873 Women taking PTU during the $1^{\text {st }}$ trimester of pregnancy according to

2874 recommendations 80,83 or 87 may be switched to MMI at the beginning of the $2^{\text {nd }}$

2875 trimester, or they may continue PTU therapy for the remaining part of pregnancy if

2876 ATD is needed. No Recommendation, insufficient evidence to assess benefits and $2877 \quad$ risks.

The reason for the FDA black box warning against PTU therapy after the $1^{\text {st }}$

2879 trimester of pregnancy is the risk of PTU associated liver failure. However, even if this

2880 risk is real, the absolute risk observed in studies of US health databases was low

2881 (433,465). Similarly, a recent Danish national registry study observed one case of

2882 reversible liver failure among 1,103 women treated with PTU in pregnancy (129).

The risk of side effects from PTU should be weighed against the risk of the shift

2884 from PTU to MMI inducing a transient thyroid function abnormality in the pregnant

2885 woman who is well controlled on PTU therapy. Starting from the $2^{\text {nd }}$ trimester of

2886 pregnancy, women with GD may start entering gradual remission of the autoimmune

2887 abnormality, and full focus should be on the feasibility of ATD dose reduction to protect

2888 the fetus against goiter and hypothyroidism, as discussed below. Patients who remain on 
2889 PTU during the $2^{\text {nd }}$ and $3^{\text {rd }}$ trimesters could have hepatic enzymes measured at the same 2890 time that thyroid function is assessed. However, no prospective data show that this type 2891 of monitoring is effective in preventing fulminant PTU-related hepatotoxicity. Another 2892 aspect to consider is that both agranulocytosis and liver failure developing during MMI 2893 and PTU therapy mostly occur during the initial three months of therapy (128), but this 2894 risk can recur when the drug is reintroduced after a relatively long period of time (177).

2895 For example, in a Japanese study (177) of 14 patients who developed agranulocytosis 2896 after retreatment with the same ATD, no patient developed this adverse reaction who 2897 restarted the drug less than 5 months after stopping the previous course of therapy. There 2898 are no data to directly evaluate how shifting from PTU to MMI in the $2^{\text {nd }}$ trimester of 2899 pregnancy will affect the risk of these severe, but rare side effects.

Other medical treatments for hyperthyroidism during pregnancy

Other types of medical therapy have been used to treat hyperthyroidism, such as

2902 iodine, perchlorate, cholestyramine, cholecystographic agents, and lithium.

2904 thyroid, and it has with some success been used to treat hyperthyroid women in

2905 pregnancy in Japan. In one study, cord and maternal sera were tested at delivery in 35

2906 patients with GD treated with iodine (6-40 mg daily) initiated at 11-37 weeks of

2907 gestation. Similar to ATD therapy, thyroid function at term tended to be lower in the

2908 fetus than in the mother, but overall results of therapy were judged satisfactory, with a

2909 low risk of inducing hypothyroidism and goiter in the fetus; only 1 of 35 neonates had

2910 subclinical hypothyroidism at birth (483). In a recent study, outcomes of pregnancy 
2911 were retrospectively compared in 1,333 women who had continued ATD in early

2912 pregnancy with 283 women who had shifted from ATD to iodine (median gestational

2913 week of shift was week 6 (range 4-12)) (476). Overall, shifting had been more common

2914 in recent years. The prevalence of major birth defects was lower in the women who had

2915 shifted to iodine therapy ( $1.53 \%$ versus $4.14 \%, \mathrm{p}<0.05)$. However, according to the

2916 authors, some degree of hyperthyroidism was relatively common after shifting, and free

$2917 \mathrm{~T}_{4}$ levels were always higher in the group that had shifted to iodine. Despite this, live

2918 births were more common in the group that had shifted than in the group that had

2919 continued MMI therapy (91.9\% versus $85.1 \%, \mathrm{p}<0.05)$. In the publication, data on

2920 thyroid function in the MMI group are sparse, but the study may indicate that a brief

2921 period of mild hyperthyroidism in the mother will not impair pregnancy.

No recent data on iodine therapy for GD in pregnancy are available from outside

2923 Japan, but before ATDs became available, experience with iodine therapy for GD in

2924 general was extensive (484), and it corresponds to the more recent Japanese studies. The

2925 minimal effective dose of iodine was around $6 \mathrm{mg}$ day but most patients received higher

2926 doses, iodine was effective for therapy of hyperthyroidism in patients with mild GD, but

2927 clearly less effective than ATD in patients with more severe disease (484). Additional

2928 data are needed before iodine therapy of pregnant women with GD can be generally

2929 recommended.

Perchlorate is a competitive inhibitor of iodine uptake by the thyroid, and a few

2931 cases have been published where it was used in pregnancy (485). Apparently,

2932 teratogenicity of perchlorate has not been demonstrated (486), but more clinical studies 
on this are clearly needed. Further, this drug is not available in the United States.

2937 to affect the fetus directly. However, binding in the gut and excretion of vitamins and

2938 other substances of importance for pregnancy is a concern, and has led to a note of

2939 caution by the US Food and Drug Administration. Cholecystographic drugs are not

2940 generally available any more. Lithium may be teratogenic (489) and it should not be used

2941 to treat hyperthyroidism in pregnancy.

\section{- RECOMMENDATION 89}

2944 GD during pregnancy should be treated with the lowest possible dose of ATD needed

2945 to keep the mother's thyroid hormone levels at or slightly above the reference range

2946 for total $\mathrm{T}_{4}$ and $\mathrm{T}_{3}$ values in pregnancy (1.5 times above non-pregnant reference

2947 ranges in the $2^{\text {nd }}$ and $3^{\text {rd }}$ trimester), and the TSH below the reference range for

2948 pregnancy. Similarly, free $\mathrm{T}_{4}$ levels should be kept at or slightly above the upper limit

2949 of the pregnancy trimester reference range for the assay. Thyroid function should be

2950 assessed at least monthly, and the ATD dose adjusted, as required. Strong

2951 recommendation, low-quality evidence.

Even if the mother is euthyroid during ATD therapy, there is a risk of inducing

2953 fetal hypothyroidism and goiter during the second and third trimesters when the fetal 
2954 thyroid has begun to function $(490,491)$. Thus, the dose of ATD should be kept as low as 2955 possible. Block-replacement therapy consisting of ATD plus levothyroxine should not be 2956 used in pregnancy. If a woman receiving such therapy becomes pregnant, and she is still 2957 in need of ATD therapy, the regimen should be changed to an ATD alone (444).

Technical remarks: Free $\mathrm{T}_{4}$ is the parameter that has been most closely correlated 2959 with good fetal outcome. Serum TSH may still be suppressed in these patients and should 2960 not be used as the sole guide in treatment, although normalization of maternal TSH 2961 during ATD therapy may indicate a need to reduce the dose of ATD (444). In Japanese 2962 studies, ATD treated maternal free $\mathrm{T}_{4}$ values had been kept above the non-pregnancy 2963 reference range in the last part of pregnancy to avoid cases of elevated TSH in newborn 2964 cord blood $(458,491)$. However, with some automated free $\mathrm{T}_{4}$ assays non-pregnancy free $2965 T_{4}$ is much higher than late pregnancy free $T_{4}(446,492)$. Thus, maternal free $T_{4}$ above the 2966 non-pregnancy reference with suppressed TSH may leave the mother overtly 2967 hyperthyroid, which is not recommended. Although many patients with GD may enter remission of the autoimmune

2969 abnormality during the $2^{\text {nd }}$ half of pregnancy with a need of ATD dose reduction or 2970 withdrawal, this is not a universal phenomenon. A small group of patients suffers from 2971 severe disease that may even progress during pregnancy, with difficult to treat 2972 hyperthyroidism, high TRAb levels and often a considerable goiter with high blood flow. 2973 Such patients may show a 'high $\mathrm{T}_{3}-$ low $\mathrm{T}_{4}$ pattern' during ATD therapy (444)

2974 presumably caused by a high type 1 deiodinase activity in the hyperactive thyroid (493), 2975 and preferential $\mathrm{T}_{3}$ synthesis in the hyperstimulated thyroid made iodine deficient from 
2976 ATD therapy (494). Maternal thyroid function should be monitored frequently and non-

2977 invasive assessment of fetal thyroid function (e.g. fetal heart rate, bone maturity,

2978 and fetal goiter on ultrasound), and ATD therapy balanced to keep acceptable thyroid

2979 function in both the mother and the fetus (444).

2980 - RECOMMENDATION 90

2981 Pregnancy is a relative contraindication to thyroidectomy and should only be used

2982 when medical management has been unsuccessful or ATDs cannot be used. Strong

2983 recommendation, low-quality evidence.

In a population based US study, pregnant women had worse clinical and

2985 economic outcomes following thyroid (and parathyroid) surgery than non-pregnant

2986 women, with disparities in outcomes based on race, insurance, and access to high-volume

2987 surgeons (68).

\section{- RECOMMENDATION 91}

2989 When thyroidectomy is necessary for the treatment of hyperthyroidism during

2990 pregnancy, the surgery should be performed if possible during the second trimester.

2991 Strong recommendation, low-quality evidence.

Thyroidectomy is best avoided in the first and third trimesters of pregnancy

2993 because of teratogenic effects associated with anesthetic agents and increased risk of fetal

2994 loss in the first trimester and increased risk of preterm labor in the third. Optimally, 
2995 thyroidectomy would be performed in the latter portion of the second trimester. Although

2996 it is the safest time, it is not without risk $(4.5-5.5 \%$ risk of preterm labor) $(67,68)$.

2997 Evaluation by a high-risk obstetrician is advised along with counseling before surgery

2998 regarding risks involved (68). Thyroidectomy cures the hyperthyroidism and is often

2999 followed by a gradual reduction in circulating TRAb (495). Until such remission takes

3000 place, TRAb produced by the mother may stimulate the thyroid of the fetus or newborn

3001 and induce hyperthyroidism. In the setting where the mother still harbors TRAb after

3002 thyroidectomy, close fetal monitoring for both cardiovascular and skeletal changes with

3003 fetal ultrasound is essential.

3004 There are no data concerning whether SSKI or iodine should be used to prepare

3005 pregnant patients for thyroidectomy. The risk of iodide therapy to the fetus relates to

3006 inhibition of iodine organification via the Wolff-Chaikoff effect. The fetal thyroid gland

3007 is particularly susceptible to the inhibitory effects of excess iodine in the second half of

3008 gestation, and fetal goiter can occur with chronic therapy (496). However, there is no

3009 evidence that brief iodine preparation of the mother done preoperatively to reduce thyroid

3010 blood flow and control hyperthyroidism is harmful to the fetus.

3011 Technical remarks: In patients with difficult to treat hyperthyroidism,

3012 preoperative preparation for thyroidectomy during the second trimester of pregnancy

3013 includes 10 days of iodine (e.g., SSKI 1 drop three times a day), along with ATD therapy

3014 and beta-blockers (propranolol or metroprolol, but not atenolol $(450,451)$ ) to control

3015 hyperthyroidism (497-499). In euthyroid patients with no signs of high thyroid activity, 
3016 but who are offered surgical thyroidectomy for other reasons, e.g. intolerance to ATD,

3017 the use of iodine for surgical preparation is considered unnecessary.

3018 [T3] The role of TRAb levels measurement in pregnancy

\section{- RECOMMENDATION 92}

TRAb levels should be measured when the etiology of hyperthyroidism in pregnancy

3021 is uncertain. Strong recommendation, low-quality evidence.

The two best indicators of the activity of GD during pregnancy are thyroid

3023 function in the untreated patient and measurement of TRAb levels in the serum. TRAb

3024 measurement is useful in the diagnosis of GD in pregnant women with newly diagnosed

3025 hyperthyroidism who do not have clinical signs specific for GD, keeping in mind that the

3026 diagnostic sensitivity of good assays is around 95\%, and the specificity is 99\% (47).

\section{7 - RECOMMENDATION 93}

3028 Patients who were treated with RAI or thyroidectomy for GD prior to pregnancy

3029 should have TRAb levels measured using a sensitive assay initially during the first

3030 trimester thyroid function testing and, if elevated, again at 18-22 weeks of gestation.

3031 Strong recommendation, low-quality evidence.

Measurement of TRAb levels can detect persistent TSH-receptor autoimmunity in

3033 a pregnant woman previously treated with ablative therapy (radioactive iodine or

3034 thyroidectomy) for GD who is now euthyroid with or without thyroid hormone

3035 replacement $(495,500)$. If the mother still produces TRAb, the antibodies will cross the 
3036 placenta and may affect fetal thyroid function in the last half of the pregnancy. Because

3037 of the slow clearance of maternal immunoglobulin $\mathrm{G}(\mathrm{IgG})$ from the neonatal circulation,

3038 thyroid dysfunction in the child may last for several months after birth. To evaluate the

3039 risk of such complications, a TRAb level should be measured in the pregnant woman

3040 initially during the first trimester and, if elevated, again at 18-22 weeks of gestation. If

3041 the level is high, a program of fetal and neonatal surveillance for thyroid dysfunction

3042 should be initiated (501).

3043 The advantage to initial TRAb measurement during the first trimester is that this

3044 allows time to initiate specialty consultation and, if the levels are especially high at that

3045 time, intervention may be required by the $2^{\text {nd }}$ trimester. Whereas it has generally been

3046 considered that isolated fetal thyrotoxicosis in a previously ablated mother who is still

3047 producing TRAb might only start developing around weeks 20-22 of pregnancy, a recent

3048 case report described severe fetal thyrotoxicosis that had developed already in gestational

3049 week 18 (502). The pregnant women had previously undergone unsuccessful RAI, and

3050 subsequently a total thyroidectomy had been performed followed by $\mathrm{L}-\mathrm{T}_{4}$ replacement.

3051 The mother was now euthyroid, but her TRAb values remained extremely elevated.

$\mathrm{TRAb}$ measurement is not necessary in a euthyroid pregnant patient previously

3053 found to have GD if she has an intact thyroid (i.e., not previously treated with surgery or

3054 RAI) and she is not currently taking ATDs $(495,503)$. 
3056 Patients receiving ATD for GD when becoming pregnant or found to have GD during 3057 pregnancy should have TRAb levels measured at initial pregnancy visit or at diagnosis 3058 using a sensitive assay and, if elevated, again at 18-22 weeks of gestation. Strong recommendation, low-quality evidence. TRAb (TBII or TSI) measurement may be useful to assist in the evaluation of

3061 disease activity in a woman being treated with ATDs for GD during pregnancy

3062 (444,495). In many patients, GD gradually remits during pregnancy. Disappearance of

3063 TRAb is an indication that ATD therapy may no longer be necessary, and that its

3064 continuation may put the fetus at risk for hypothyroidism, even if the mother is euthyroid 3065 on the medication.

\section{- RECOMMENDATION 95}

Patients with elevated TRAb levels at 18-22 weeks of gestation should have

3068 TRAb remeasured in late pregnancy (weeks 30-34) to guide decisions regarding neonatal

3069 monitoring. An exception to this is a woman with an intact thyroid who is no longer in

3070 need of ATD therapy. Strong recommendation, low-quality evidence.

$\mathrm{TRAb}$ measurement in late pregnancy can be used to assess the risk of delayed

3072 neonatal hyperthyroidism, when the mother continues to need ATD to control

3073 hyperthyroidism up to term. After delivery, ATD delivered to the fetus via placental

3074 passage is rapidly metabolized by the neonate, whereas the maternal TRAb disappears

3075 more slowly, with a half-life of around 3 weeks. Thus, a high level of TRAb in the

3076 mother in late pregnancy is an indicator that the neonate may need to be monitored for 
3077 the onset of neonatal hyperthyroidism starting a few days after birth. In a recent study of 307847 newborns to mothers who were TRAb positive in pregnancy, nine of the children had 3079 neonatal biochemical hyperthyroidism, and five of these (9\% of all) needed ATD

3080 therapy. All hyperthyroid neonates were born to mothers with TRAb levels $\geq 5$ IU/1 (> 3

3081 times upper reference for the assay) in the $2^{\text {nd }}$ trimester (sensitivity $100 \%$, specificity $308243 \%$ ). All mothers who gave birth to hyperthyroid newborns required ATD therapy in 3083 late pregnancy (504).

3084 [T4] Postpartum thyroiditis

3085 - RECOMMENDATION 96

3086 In women developing thyrotoxicosis after delivery, selective diagnostic studies should 3087 be performed to distinguish postpartum destructive thyroiditis from postpartum GD.

3088 Strong recommendation, low-quality evidence.

3090 States. Postpartum thyroiditis is an autoimmune disorder unmasked in predisposed

3091 women as immune surveillance rebounds after pregnancy. The classic triphasic pattern is

3092 thyrotoxicosis at 1-6 months postpartum, followed by hypothyroidism and return to

3093 euthyroidism at 9-12 months postpartum $(505,506)$. However, this sequence is not

3094 observed in every patient. Among 371 cases in 13 studies, 25\% of patients were found to

3095 have a triphasic pattern, $43 \%$ had hypothyroidism without preceding thyrotoxicosis, and $309632 \%$ had thyrotoxicosis without subsequent hypothyroidism (506). In a prospective study 3097 of pregnant women, those with positive thyroperoxidase (TPO) antibodies in the first 
3098 trimester were 27 times more likely to develop postpartum thyroiditis than were those

3099 with negative serology (507). In this study, tobacco smoking and bottle-feeding increased

3100 the risk of developing thyroiditis.

3101 Postpartum thyroiditis must be distinguished from GD to recommend proper

3102 therapy. The postpartum surge in thyroid autoimmunity leading to postpartum thyroiditis

3103 is also associated with a 3-4 fold increase in the incidence of GD that peaks 3-12 months

3104 after delivery (456). In a Japanese hospital study, thyrotoxicosis caused by thyroiditis

3105 developed earlier post-partum than GD, although some overlap existed. All patients who

3106 developed overt thyrotoxicosis within the first three months after delivery suffered from

3107 destructive thyroiditis, whereas GD developed after this 3 months period (508). Goiter is

3108 generally more pronounced in GD, and thyroid bruit or GO strongly suggest GD as well.

3109 TRAb may occasionally be measurable in patients with postpartum thyroiditis,

3110 suggesting that some patients may suffer from a combination of GD and destructive

3111 thyroiditis (509), but higher TRAb values are suggestive of GD. When in vivo testing is

3112 required to make this distinction in women who are nursing, the gamma-emitters 123-I

3113 (half-life 13 hours) or Tc-99m- pertechnetate (half-life 6 hours) should be used rather

3114 than the beta-emitter 131-I (half-life 8 days). The shorter half-lives of these agents (510)

3115 will allow breast milk to be pumped and discarded for 10 half-lives ( 5 or 3 days

3116 respectively) and nursing resumed, whereas breast-feeding should ideally be discontinued

31173 months prior to 131-I to avoid radiation exposure to the breast, and not be resumed if

3118 131-I is given as treatment for GD (511). 
3119 Most often, the use of radioactive substances can be avoided and the diagnosis

3120 can be based on a combination of clinical presentation, TRAb measurement, and

3121 evaluation of serum $T_{4}$ and $T_{3}$. Thyroidal production of $T_{3}$ compared with $T_{4}$ is relatively

3122 high in GD, but not in destructive thyroiditis, and $\mathrm{T}_{3}$ tends to be fractionally more

3123 elevated above the upper reference limit than $\mathrm{T}_{4}$ in GD, whereas $\mathrm{T}_{4}$ is more elevated than

$3124 \mathrm{~T}_{3}$ in destructive thyroiditis (50). If needed, thyroid color doppler ultrasonography may

3125 assist to distinguish between destructive thyroiditis and GD $(508,512,513)$.

3126 - RECOMMENDATION 97

3127 In women with symptomatic thyrotoxicosis from postpartum destructive thyroiditis,

3128 the judicious use of beta-adrenergic blocking agents is recommended. Strong

3129 recommendation, low-quality evidence.

3130 Treatment for postpartum thyroiditis is generally supportive in nature, with the

3131 use of beta-adrenergic blockers such as propranolol or metroprolol to control pulse rate

3132 and hyperadrenergic symptoms during the thyrotoxic stage (514). The selective beta-1

3133 adrenergic receptor-blocking agent atenolol should not be used in breast-feeding mothers

3134 because this may lead to symptoms consistent with beta-adrenergic blockage in neonates.

3135 This adverse effect presumably develops because atenolol is $<5 \%$ bound to maternal

3136 plasma proteins (vs. $93 \%$ binding of propranolol), and thus accumulates in milk, and

3137 because of low kidney excretion of atenolol in small children with immature renal

3138 function (515). Levothyroxine therapy may be beneficial, at least transiently, for women

3139 with symptomatic hypothyroidism or having TSH levels $>10 \mathrm{mU} / \mathrm{L}$ (506). 
3141 milk in only very low levels, no special monitoring is needed for breastfed infants of

3142 mothers on these medications (514).

\section{3 - RECOMMENDATION 98}

3144 In pregnant women diagnosed with hyperthyroidism due to multinodular thyroid

3145 autonomy or a solitary toxic adenoma special care should be taken not to induce fetal

3146 hypothyroidism by ATD therapy. Strong recommendation, low-quality evidence.

3147 Hyperthyroidism caused by thyroid autonomy is very common in people having 3148 current (or previous) mild to moderate iodine deficiency (13), but it mostly develops in 3149 patients after the age of 50 years. In the uncommon case of this type of hyperthyroidism 3150 in a pregnant woman, pathogenic differences from GD should be considered.

Thyroid hormone production in autonomy is dependent on iodine substrate,

3152 but no study has addressed the effect of a change in iodine intake on thyroid

3153 function in pregnant women with autonomy, or on the fetus. It might be beneficial

3154 to keep iodine intake on the low side, but care must be taken that the fetus is not

3155 iodine deficient, especially in areas where the population is iodine deficient. The

3156 degree of maternal hyperthyroidism and assessment of her diet should be

3157 considered before deciding whether to administer iodine supplements. Often the

3158 hormone overproduction is limited in patients with autonomy (50). In mild cases there

3159 would be a theoretical possibility that the normal pregnancy associated increase in

3160 thyroid hormone production may catch up with the hormone production in the 
3161 autonomous areas of the thyroid, and alleviate the need for ATD therapy. On the other

3162 hand, the high hCG levels in early pregnancy may theoretically stimulate the non-

3163 functioning normal thyroid tissue in these patients and worsen hyperthyroidism. Because

3164 there is no TRAb production, the fetal thyroid will not be abnormally stimulated in the

3165 second half of pregnancy as it is in GD. Thus, the fetus will not develop hyperthyroidism

3166 in parallel with the untreated hyperthyroid mother as it happens during $2^{\text {nd }}$ half of

3167 pregnancy in GD, and neonatal hyperthyroidism is not a risk. On the other hand, the

3168 tendency to induce fetal hypothyroidism and goiter in the $2^{\text {nd }}$ half of pregnancy from

3169 ATDs given to the mother would be even higher in this type of hyperthyroidism than in

3170 GD. Based on this theoretical risk, surgical therapy in the $2^{\text {nd }}$ trimester of pregnancy may

3171 be considered if the hyperthyroidism turns out to require more than low dose MMI (5-10

3172 mg per day) for control. No firm recommendations are given, because no good evidence

3173 is available.

[U] How should hyperthyroidism be managed in patients with Graves' orbitopathy?

GO is an inflammatory eye disease that develops in the orbit in association with

3177 autoimmune thyroid disorders (516). In the majority of cases (about 90\%), it occurs in

3178 patients with current or past GD. Thyroid-associated orbitopathy, thyroid eye disease, and

3179 Graves' ophthalmopathy are other names used for GO. Approximately a third of patients

3180 with Graves' hyperthyroidism have some signs and/or symptoms of GO while only 5\%

3181 suffer from moderate-to-severe disease $(517,518)$. In contrast to GD where women are at

3182 higher risk, the role of gender in GO is more controversial. More recent studies do not 
3183 identify a clear gender related-risk for GO $(517,518)$, while some older studies point to a 3184 possible slightly increased risk for men $(519,520)$. This variability in results might be 3185 related to changes in smoking patterns over the years. The disease peaks in incidence in 3186 the $5^{\text {th }}$ and $6^{\text {th }}$ decade of life $(517,518,521,522)$ with a higher prevalence of severe cases 3187 in the elderly population (517)

[U1] Assessment of disease activity and severity The natural history of the disease is one of rapid deterioration followed by gradual

3190 improvement toward the baseline. This active phase is best described by the Clinical

3191 Activity Score (CAS) $(523,524)$, the elements of which are outlined in Table 12 . The 3192 score ranges from 0 to 10 and predicts response to anti-inflammatory therapies $(523,524)$. 3193 A 7-point scale, lacking the last three elements, is used when no previous assessment is 3194 available. GO is considered active in patients with a CAS $\geq 3$. However, some of the eye 3195 changes seen in hyperthyroidism, like lid retraction or stare, are the result of the increased 3196 sympathetic state and when present without associated eye changes are not considered to 3197 reflect GO (69).

The severity of the disease is best assessed using objective, quantifiable

3199 parameters and is a useful tool for directing therapy. The main gradations of disease 3200 severity are mild, moderate-to-severe, and sight threatening (525). Table 13 lists the 3201 elements as agreed upon in a consensus statement by the European Group on Graves'

3202 Orbitopathy (EUGOGO) (525). Both activity and severity of the disease must be 3203 considered in therapeutic decisions regarding treatment of the eye disease itself, as well 3204 as treatment of hyperthyroidism, keeping in mind that they do not always correlate, 
3205 particularly in early and late disease. The overall evaluation and management of GO is

3206 best done in a multidisciplinary clinic combining endocrinologists and ophthalmologists

3207 with expertise in the condition and other specialties in consultation (e.g., ENT, radiation

3208 therapy, plastic surgery, and endocrine surgery).

3209 Quality of life is clearly impaired by GO (526). The U.S. Food and Drug

3210 Administration has endorsed QoL information as a component of any therapeutic

3211 application. The QoL correlation with disease severity has been fair to excellent for two

3212 GO specific instruments published to date in North American populations $(527,528)$,

3213 though the effect of GO therapy on these QoL scores still needs prospective data.

3214 Presently the only instrument that has such data is the instrument extensively used in

3215 Europe (529) that has not yet been tested in a North American population. Overall this

3216 area is in need of more research emphasis as despite its agreed upon importance a

3217 significant number of intervention trials in GO are still being reported without associated

3218 QOL outcomes (530).

3219 In the remainder of section [U], we discuss the prevention of GO and the

3220 management of hyperthyroidism in patients having established GO. In particular, we

3221 focus on recommendations regarding the concurrent use of corticosteroids in patients

3222 choosing RAI as treatment for hyperthyroidism (Table 14).

3223 [U2] Prevention of GO

3224 Current therapeutic approaches to GO, including local measures, corticosteroids, 3225 orbital radiation, and surgery (525), often fail to significantly improve the QoL of 
3226 patients with this debilitating condition. Therefore, efforts should be made to prevent the

3227 development or progression of GO in patients with Graves' hyperthyroidism. Identified

3228 risk factors for GO are listed in Table 15 and most pertinent to this discussion are RAI

3229 therapy for hyperthyroidism $(531,532)$, untreated hyperthyroidism, smoking, high serum

3230 pretreatment TRAb levels (normal $<1.75 \mathrm{IU} / \mathrm{L}$, high risk for progression if $>8.8$

3231 IU/Liter) (533), and any delay in treating hypothyroidism after therapy for

3232 hyperthyroidism (106,534). High pretreatment levels of $\mathrm{T}_{3}$ and $\mathrm{T}_{4}$ were each reported

3233 to have a predictive role in GO but these conclusions were not validated by subsequent

3234 studies $(69,106,532,534)$ suggesting the possibility of higher TRAb values, measured on

3235 less sensitive assays early-on, being partly responsible for this variation.

3236 - RECOMMENDATION 99

3237 Euthyroidism should be expeditiously achieved and maintained in hyperthyroid

3238 patients with GO or risk factors for the development of orbitopathy. Strong

3239 recommendation, moderate-quality evidence.

3240 A number of studies have suggested that development of persistent, untreated

3241 hypothyroidism after therapy for hyperthyroidism plays a detrimental role in the

3242 progression of GO. An early study noted that patients who were either hypo- or

3243 hyperthyroid had more severe GO than euthyroid patients (535). Subsequently, two

3244 cohort studies in which patients received levothyroxine therapy early after RAI with the

3245 specific intent of preventing hypothyroidism noted that deterioration of GO rarely

3246 occurred $(0 \%-2 \%)(534,536)$. A randomized study of newly diagnosed GD found that

3247 RAI, followed by active prevention of hypothyroidism by administration of thyroid 
3248 hormone 2 weeks later, did not increase the risk of worsening GO compared to therapy

3249 with MMI (RR of 0.95) (69).

3251 We recommend clinicians advise patients with GD to stop smoking and refer them to a

3252 structured smoking cessation program. As both firsthand and secondhand smoking

3253 increase GO risk patients exposed to secondhand smoke should be identified and

3254 advised of its negative impact. Strong recommendation, moderate- quality

3255 evidence.

Smoking is the most important known risk factor for the development or

3257 worsening of GO, unrelated to type of therapy for GO (535), and consistent data from

3258 several studies show a detrimental effect of smoking on GO in patients treated with

3259 RAI $(69,531)$. The risk is proportional to the number of cigarettes smoked per day and

3260 former smokers have significantly lower risk than current smokers, even after adjusting

3261 for lifetime cigarette consumption (537).

3262 Technical remarks: Clinicians should use smoking cessation programs based on

3263 effective and evidence-based approaches to aid in smoking cessation and avoidance of

3264 secondhand smoke $(538,539)$.

$3265[\mathrm{U} 3]$ Treatment of hyperthyroidism in patients with no apparent GO 

concurrent steroids), ATDs or thyroidectomy should be considered equally acceptable

3269 therapeutic options in regard to risk of GO. Strong recommendation, moderate3270 quality evidence.

3271 Several randomized trials have identified the risk of GO development or 3272 progression after RAI therapy for hyperthyroidism to be between $10 \%$ and $39 \%(69,540)$.

3273 Focusing on the risk of new GO development that risk appears to be lower. Two

3274 randomized controlled trials found that risk to be $6 / 78(8 \%)$ for RAI, compared with 1/74 3275 (1\%) for ATDs (531) in one study, and 10/32 (32\%) for RAI compared with $6 / 56(11 \%)$ 3276 for ATDs and 6/58 (10\%) for surgery (532) in the older study. Fortunately, the cases of 3277 new or worse GO are usually mild with only $6 / 168$ patients on this second trial (4 in the 3278 RAI group, 1 in the ATD group and 1 in the surgical group) requiring specific therapy for 3279 GO. In contrast, one prospective but nonrandomized cohort study identified no difference 3280 among ATD, surgery, and RAI treatment, with an overall 4.9-7.1\% frequency of GO 3281 development (541). The higher risk of GO development after RAI therapy in the majority 3282 of studies may be related to the unique increase in TRAb levels observed following this 3283 therapy (172). Experimental evidence suggests that these antibodies are directly involved 3284 in GO pathogenesis $(516,542,543)$. There is evidence that corticosteroids given concurrently with RAI may prevent 3286 worsening of GO in patients with mild active eye disease (531). However, there is 3287 insufficient evidence to recommend prophylactic treatment with corticosteroids in 3288 nonsmoking patients who do not have clinically apparent GO. The relatively low absolute 
3289 risk of nonsmokers developing new-onset severe GO suggests that GO prevention should

3290 not be a factor in the selection of therapy for hyperthyroidism in this group of patients

3291 (531). Table 14 details further the use of glucocorticoids for various GO clinical

3292 scenarios.

\section{- RECOMMENDATION 102}

3294 In smoking patients with GD without apparent GO, RAI therapy, ATDs, or

3295 thyroidectomy should be considered equally acceptable therapeutic options in regard

3296 to risk of GO. Weak recommendation, low-quality evidence.

\section{7 - RECOMMENDATION 103}

3298 There is insufficient evidence to recommend for or against the use of prophylactic

3299 corticosteroids in smokers who receive RAI and have no evidence of GO. No

3300 recommendation, insufficient evidence.

$3301 \quad$ However, in two different studies, active smokers who received RAI represented

3302 the group with the highest incidence (23-40\%) of new GO or deterioration of pre-

3303 existing GO during 1 year of follow-up $(69,531)$.

3304 [U4] Treatment of hyperthyroidism in patients with active GO of mild severity (see

3305 Tables 12 and 13 for definitions of disease activity and severity) 
3307 In patients with Graves' hyperthyroidism who have mild active ophthalmopathy and

3308 no risk factors for deterioration of their eye disease, RAI therapy, ATDs and

3309 thyroidectomy should be considered equally acceptable therapeutic options. Strong

3310 recommendation, moderate-quality evidence.

\section{1 - RECOMMENDATION 105}

3312 In the absence of any strong contraindication to GC use we suggest considering them

3313 for coverage of GD patients with mild active GO who are treated with RAI, even in

3314 the absence of risk factors for GO deterioration. Weak recommendation, low-quality

3315 evidence.

3316 Technical remarks: The decision whether or not to administer concurrent

3317 glucocorticoids in a particular patient choosing RAI therapy should be made in light of

3318 risk-benefit considerations (i.e., their personal risk of worsening GO, balanced against

3319 their risk of developing glucocorticoid side effects). Risk factors for side effects of oral

3320 corticosteroids include poorly controlled diabetes, hypertension, osteoporosis, psychiatric

3321 disease, and predisposition to infections. Smokers in whom the risk-benefit ratio for the

3322 concurrent use of corticosteroids is high may be better treated with ATDs or surgery.

3323 Besides smoking, the main risk factors for deterioration of GO to be considered in this

3324 decision include active and progressive GO over the preceding 3 months and high serum

3325 pretreatment TRAb levels (normal $<1.75 \mathrm{IU} / \mathrm{L}$, high risk for GO progression if $>8.8$

3326 IU/Liter) (see Table 15). 
3328 prophylaxis is the equivalent of prednisone $0.4-0.5 \mathrm{mg} / \mathrm{kg} / \mathrm{day}$, started $1-3$ days after

3329 RAI administration, continued for 1 month, and then tapered over 2 months (525).

3330 However, a retrospective cohort study suggested that even lower doses and shorter

3331 duration of oral prednisone (about $0.2 \mathrm{mg} / \mathrm{kg}$ / day for 6 weeks) may be equally effective

3332 for prevention of GO exacerbation in patients with initially mild or absent eye disease,

3333 (544). Currently most task force members use a minimum starting dose of $30 \mathrm{mg}$

3334 prednisone daily and tapering to off within 6-8 weeks. Table 14 details further the use of 3335 glucocorticoids for various GO clinical scenarios.

\section{6 - RECOMMENDATION 106}

3337 In GD patients with mild GO who are treated with RAI we recommend steroid $3338 \quad$ coverage if there are concomitant risk factors for GO deterioration. Strong 3339 recommendation, moderate-quality evidence.

3340 Unfortunately, the initial data regarding the impact of various GD therapies on

3341 GO outcome were impacted by the absence of GO activity assessment and lack of 3342 stratification on smoking status at randomization as well as by variation in the timing of 3343 tackling post-RAI hypothyroidism. Two early nonrandomized studies found no

3344 differences between the three GD therapeutic modalities $(541,545)$.

The first randomized study of GD patients (13\% with mild preexistent GO)

3346 assigned to therapy for hyperthyroidism with antithyroid drugs, surgery or RAI (532)

3347 found the relative risk for deterioration of eye disease to be elevated at 3.2 for RAI 
3348 compared to ATDs. There appeared to be no difference in such risk between ATDs and

3349 surgery. A more recent large randomized controlled trial studying mainly patients with

3350 previously treated GD showed RAI therapy to be associated with an increased risk of GO

3351 progression (RR of 5.8 in comparison with ATDs) and found the risk to be eliminated

3352 with concurrent corticosteroid administration (531). Finally, the most recent randomized

3353 controlled trial (69) revealed the increased risk for new or worse GO in RAI treated

3354 patients (38.7\% of the group) compared with ATD-treated patients (21.3\% of the group),

3355 to be mainly related to development of new GO cases while worsening of preexistent GO

3356 occurred in a similar percentage in both groups ( $45 \%$ for RAI and $47 \%$ for ATD).

3357 Smoking was a strong risk factor for an undesirable GO outcome. In this last trial there

3358 was no routine use of prophylactic glucocorticoids. Table 14 details further the use of

3359 glucocorticoids for various GO clinical scenarios.

3360 [U5] Treatment of hyperthyroidism in patients with active and moderate-to-severe or

3361 sight-threatening GO (see Tables 12 and 13 for definitions of disease activity and

3362 severity)

3363 - RECOMMENDATION 107

3364 In patients with active and moderate to severe or sight-threatening GO we recommend 3365 against RAI therapy. Surgery or ATDs are preferred treatment options for GD in these 3366 patients. Strong recommendation, low-quality evidence.

3367 We are aware of no trials in patients with moderate-to-severe and active eye 3368 disease that compare hyperthyroidism therapies for impact on GO. However, a 
3369 comparison of two different surgical approaches (total thyroidectomy vs. subtotal

3370 thyroidectomy) for patients with moderate-to-severe GO showed that the eye disease

3371 improved over 3 years of follow-up in all patients (546). In another series of 42 patients

3372 with progressive GO treated with total thyroidectomy, exophthalmos was stable in $60 \%$

3373 of cases and improved in the remainder (547), suggesting that surgery is not detrimental

3374 to GO and may be associated with improvement in some patients. Additionally, a more

3375 recent study suggests that surgery might lead to a more rapid improvement in GO than

3376 ATDs and it might thus be a better option for patients that are most concerned about GO

3377 changes (548). Other studies suggest that ATDs may not adversely impact mild active

3378 GO, but do not address severe GO (531).

3379 Alternatively, if ATDs are selected for GD therapy there is reassuring data that 3380 long term use is relatively safe and effective at preserving euthyroidism while waiting for 3381 GO to enter remission $(66,549)$.

3382 [U6] Treatment of GD in patients with inactive GO (see Table 12 for definition of 3383 disease inactivity)

\section{4 - RECOMMENDATION 108}

3385 In patients with inactive GO we suggest RAI therapy can be administered without 3386 steroid coverage. However, in cases of elevated risk for reactivation (high TRAb, CAS

$3387 \geq 1$ and smokers) that approach might have to be reconsidered. Weak

3388 recommendation, low-quality evidence. 
There is a low rate of GO progression or reactivation following RAI in patients

3390 with inactive GO. A series of 72 patients with inactive GO according to the CAS were

3391 treated with RAI without concurrent glucocorticoid administration (536). In those whom

3392 hypothyroidism was prevented by early thyroxine therapy, no deterioration in eye disease

3393 was reported (536). Smoking history did not impact GO outcome in this cohort. A recent

3394 trial from Japan (540) randomized patients without GO or inactive GO (i.e. CAS $<3$ or

3395 T2-weighted imaging T2SIR $\leq 1$ ) to receive either glucocorticoid prophylaxis with low

3396 dose prednisolone (on average $0.28 \mathrm{mg} / \mathrm{kg} /$ day tapered rapidly over 6 weeks) or no

3397 prophylaxis at all. The rate of disease progression in the absence of risk factors was low

3398 (4.2\%) and not impacted by glucocorticoid therapy. The presence of risk factors for GO

3399 (high thyroid stimulating antibody, $\mathrm{CAS} \geq 1$ ) increased that risk, again without a benefit

3400 from low-dose steroid prophylaxis. Ultimately most GO cases were mild and only 7 cases

3401 (2.4\%) required GO-directed therapy. Whether high-dose glucocorticoid therapy would

3402 have made a difference in these patients is not known.

3403 Another study retrospectively examined the impact of concurrent oral or

3404 intravenous glucocorticoid therapy on the development or deterioration of preexistent GO

3405 after RAI therapy for relapsing GD patients (550). They identified GO development,

3406 deterioration or reactivation in approximately $7 \%$ of patients (6/83) considered at low risk

3407 who were given no steroid prophylaxis. Only 2 of these cases had preexistent inactive

3408 GO. Despite prophylaxis, 33\% of patients considered at high risk who were treated with

3409 oral glucocorticoids had worsening of GO. However, because of the lack of clarity of this

3410 retrospective study regarding prevalence of active and inactive GO in each group and the

3411 lack of pre-specified criteria for dose and route of steroid use in those considered at risk, 
3412 we weighed this evidence less in our deliberations regarding the above recommendation.

3413 Table 14 details further the use of glucocorticoids for various GO clinical scenarios.

3415 [V] How should iodine-induced and amiodarone-induced thyrotoxicosis be managed?

$3416 \quad$ [V1] Iodine-induced hyperthyroidism

3417 - RECOMMENDATION 109

3418 Routine administration of ATDs before iodinated contrast media exposure is not 3419 recommended for all patients. Weak recommendation, low-quality evidence.

3420 Technical remarks: Patients deemed to be at high risk of developing iodine-

3421 induced hyperthyroidism or whose cardiac status is tenuous at baseline may be

3422 considered for prophylactic therapy with ATDs.

3423 Iodine-induced hyperthyroidism (the Jod-Basedow phenomenon) is uncommon in 3424 modern series, and generally self-limited, but may occasionally persist for months

3425 (551,552), and may be life-threatening (553-556). Individuals most susceptible are

3426 elderly patients with autonomously-functioning nodular goiters (557), and less

3427 commonly, patients with occult GD (558), or patients with a prior history of GD and in

3428 remission after a course of ATDs (559). Very rarely, iodine excess may trigger

3429 thyrotoxicosis in patients with a previously normal thyroid gland (560). Chronic iodine

3430 deficiency increases the prevalence of autonomous thyroid nodules and therefore iodine 
3431 repletion in this setting has historically been linked to iodine-induced hyperthyroidism 3432 (561).

Multiple observational studies have examined changes in thyroid hormone levels

3434 following a single exposure to intravenous iodinated contrast in both iodine-sufficient

3435 (562-565), and deficient (566-569) regions. A study of patients living in Boston showed 3436 that 5 of $49(10.2 \%)$ developed a suppressed TSH value 1-4 weeks following exposure to 3437 a single CT study with contrast, with only one patient developing overt hyperthyroidism 3438 (565). Additional observational studies in the United States and Japan, involving 56 and 343922 patients, respectively, found no new cases of hyperthyroidism following coronary 3440 angiography (564) or hysterosalpingography (563), whereas an Australian study from a 3441 region of iodine sufficiency found that 2 of $72(2.8 \%)$ of patients developed overt 3442 hyperthyroidism and an additional 2 developed subclinical hyperthyroidism within 8 3443 weeks of iodinated contrast exposure (562). Overall, similar rates of iodine-induced 3444 hyperthyroidism have been described in iodine deficient regions, including a study from 3445 Germany in which 2 of $788(0.25 \%)$ patients developed overt hyperthyroidism following 3446 coronary angiography (566), a New Zealand study in which subclinical hyperthyroidism 3447 developed in 2 of 102 (2\%) patients after a CT-scan with iodinated contrast (567), a study 3448 from Italy which found that $1.9 \%$ of 1752 patients undergoing coronary angiography 3449 developed a suppressed TSH with normal free $\mathrm{T}_{4}$ and $\mathrm{T}_{3}$ levels (568), and finally, a 3450 Turkish study identifying new subclinical hyperthyroidism in 5.9\% of 101 patients by 8 3451 weeks following coronary angiography (569). 
3453 exposure in patients without baseline thyroid abnormality resulted in hyperthyroidism

3454 (defined only as a suppressed TSH value) with an odds ratio of 1.98 (95\% CI, 1.08-3.60;

$3455 \mathrm{P}=.03$ ), and that 23 patients would need to be exposed before encountering one case of

3456 iodine-induced thyrotoxicosis (570). Interestingly, a recent meta-analysis including 9

3457 randomized-controlled trials and 8 observational studies involving iodine

3458 supplementation of young children and pregnant women in regions of mild-moderate

3459 iodine deficiency did not find an increased risk of thyroid dysfunction following iodine

3460 supplementation of 200-300 micrograms daily (571).

3461 In summary, iodine-induced hyperthyroidism is uncommon and generally

3462 subclinical, but can occasionally be severe. For most clinical circumstances, the

3463 likelihood of developing overt thyrotoxicosis after iodinated contrast exposure is too low

3464 to justify the risk of adverse effects associated with prophylactic ATD therapy.

\section{- RECOMMENDATION 110}

3466 Beta-adrenergic blocking agents alone or in combination with MMI should be used to

3467 treat overt iodine-induced hyperthyroidism. Strong recommendation, low-quality

3468 evidence.

3469 Treatment of iodine-induced hyperthyroidism includes avoidance of additional

3470 iodine and administration of beta-blockers alone or with ATDs, depending on the severity

3471 of hyperthyroidism and the clinical status of the patient. RAI is not an option until the

3472 iodine load has been cleared and might not be desirable given the reversibility of this 
3473 condition. Recent data suggest that urinary iodine normalizes more rapidly than

3474 previously believed, with a return to baseline urinary iodine excretion within 1-2 months 3475 in most patients $(565,572)$.

3476 Technical remarks: Dosing of MMI for iodine-induced thyrotoxicosis is 20-40

3477 mg daily, given either as a daily or twice-daily dosing. There may be relative resistance

3478 to ATD in patients with iodine-induced hyperthyroidism. Urinary iodine (a spot urine

3479 iodine adjusted for urine creatinine concentration or a 24-hour urine iodine) may be

3480 monitored to assess the rate of clearance of the iodine load.

$3481 \quad$ [V2] Amiodarone-induced thyrotoxicosis

\section{- RECOMMENDATION 111}

3483 We suggest monitoring thyroid function tests before and within the first 3 months

3484 following the initiation of amiodarone therapy, and at 3-6 month intervals thereafter.

3485 Weak recommendation, low quality evidence.

3486 Amiodarone is a drug frequently used in the treatment of refractory atrial or

3487 ventricular tachyarrhymias. Amiodarone-induced thyrotoxicosis (AIT) occurs in up to 6\%

3488 of patients taking this medication in iodine-sufficient areas of the world (573-575) and in

3489 up to $10 \%$ in iodine-deficient areas, such as parts of Europe (576). Studies evaluating the

3490 adequacy of monitoring for adverse effects from amiodarone have shown suboptimal

3491 results $(577,578)$. 
Two distinct mechanisms have been proposed in the development of AIT,

3493 including an iodine-induced form of hyperthyroidism (type 1 AIT) due to the high

3494 iodine content of amiodarone (37\% by molecular weight), and a destructive

3495 thyroiditis (type 2 AIT), due to direct toxicity of amiodarone on follicular cells. Type

34961 AIT tends to occur in patients with underlying thyroid autonomy in a nodular

3497 goiter, or GD, whereas type 2 AIT occurs as a result of direct damage or induction

3498 of apoptosis in thyrocytes by amiodarone (579-582).

- RECOMMENDATION 112

3500 The decision to stop amiodarone in the setting of thyrotoxicosis should be determined

3501 on an individual basis in consultation with the treating cardiologist, based on the

3502 clinical manifestations and presence or absence of effective alternative antiarrhythmic

3503 therapy. Strong recommendation, low-quality evidence.

3504 The need for amiodarone discontinuation is controversial because (i) this drug is

3505 frequently the only medication able to control cardiac arrhythmia, (ii) the effects of this

3506 fat soluble drug may persist for many months, (iii) amiodarone may have $\mathrm{T}_{3}$-antagonistic

3507 properties at the cardiac level and inhibit $\mathrm{T}_{4}$ to $\mathrm{T}_{3}$ conversion in the heart (583), such that

3508 withdrawal may actually aggravate cardiac manifestations of thyrotoxicosis (573). Deaths

3509 from ventricular fibrillation have occurred after stopping amiodarone in patients with

3510 AIT (584). In addition, type 2 AIT typically responds to treatment even if amiodarone

3511 therapy is continued (585-587), but continuation may lead to a more prolonged time to

3512 recovery and a higher rate of future recurrences of AIT (588). 
3514 In clinically stable patients with AIT, we suggest testing to identify disorders

3515 associated with iodine-induced hyperthyroidism (type 1 AIT), specifically including

3516 toxic nodular disease and previously occult Graves' disease. Strong

3517 recommendation, low-quality evidence.

3518 - RECOMMENDATION 114

3519 MMI should be used to treat overt thyrotoxicosis in patients with proven underlying

3520 autonomous thyroid nodules or Graves' disease as the cause of amiodarone-induced

3521 thyrotoxicosis (type 1 disease), and corticosteroids should be used to treat patients

3522 with overt amiodarone-induced thyroiditis (type 2 disease). Strong recommendation,

$3523 \quad$ low-quality evidence.

- RECOMMENDATION 115

3525 Combined ATD and corticosteroid therapy should be used to treat patients with overt

3526 amiodarone-induced thyrotoxicosis who are too unstable clinically to allow a trial of

3527 monotherapy, or who fail to respond to single modality therapy, or patients in whom

3528 the etiology of thyrotoxicosis cannot be unequivocally determined. Strong

3529 recommendation, low-quality evidence.

As the pathogenesis of AIT is not fully understood, it is likely that the classic

3532 division of AIT into two subtypes represents an oversimplification. First, as discussed 
3533 further below, many patients are not readily classified into one of the two AIT subtypes.

3534 Secondly, once classified as type 1 or type 2 AIT, patients often fail to respond to therapy 3535 specifically directed to that subtype $(583,589,590)$. Finally, findings of responsiveness of 3536 type 2 AIT patients to measures not typically useful in destructive thyroiditis, such as 3537 perchlorate $(586,591)$ and oral cholecystographic agents $(592,593)$, cannot be adequately 3538 explained on the basis of the current classification system, although spontaneous 3539 resolution independent of therapy is one possible explanation.

3540 A number of methods have been examined to distinguish type 1 from type 2 AIT, 3541 but with the possible exception of Color flow Doppler study (CFDS), most are considered 3542 unreliable (574). For example, the $\mathrm{T}_{3}$-to- $\mathrm{T}_{4}$ ratio, which tends to be higher in patients 3543 with autonomous thyroid glands than in those with destructive thyroiditis, is not helpful 3544 in this instance due to amiodarone-associated inhibition of $\mathrm{T}_{4}$ monodeiodination (594).

3545 Further, features historically used to distinguish the subtypes such as antibodies against 3546 thyroid peroxidase and the presence of thyroid nodules in patients with type 1 AIT may 3547 actually occur with both subtypes, given the prevalence of these abnormalities in the 3548 general population. Interleukin-6 levels and radioactive iodine uptake values, once 3549 promoted as useful to distinguish between subtypes (590), actually overlap extensively 3550 between the two subtypes and are therefore also not useful (594). Several modern series 3551 of patients with AIT make no attempt to classify patients into type 1 or type 2 disease $3552(585,595-598)$. Several studies have shown that increased vascularity on color-flow Doppler 3554 study may be seen in patients with type 1, but not type 2 AIT (599-601). Two studies 
3555 showed a clear separation into type 1 and type 2 AIT, allowing successful application of 3556 targeted therapy $(599,600)$. However, CFDS is not universally useful $(584,589)$ In a 3557 series of 24 cases of AIT, 12 patients were classified as type 2 due to an absence of 3558 vascularity (CFDS 0) and treated with corticosteroids, but only 7 (58\%) proved 3559 responsive (584). Likewise, these authors found that among 11 patients classified as type 35601 AIT based on CFDS scores of I-III, only 4 (36\%) responded to antithyroid drug 3561 therapy. In another series of 30 patients with AIT requiring therapy, 10 (33\%) patients 3562 could not be subtyped on the basis of CFDS, including several patients with goiters but 3563 normal vascular flow (589). In a series of 55 patients in whom a CFDS qualitative 3564 assessment of vascular flow was used to distinguish type 1 from type 2 AIT, $81.3 \%$ of 3565 patients determined to have type 1 AIT had pattern I vascularity (the lowest level above 3566 zero), illustrating the skill and nuance needed to successfully make this distinction (599). 3567 Among European thyroidologists surveyed on the use of diagnostic imaging in the 3568 differential diagnosis of AIT, approximately $20 \%$ preferred RAIU alone, $20 \%$ preferred 3569 CFDS alone, nearly $40 \%$ utilized both methods simultaneously, and $20 \%$ thought both 3570 techniques were useless (602). Recently sestamibi uptake by the thyroid, which is 3571 diminished with thyroiditis, has been applied to distinguishing AIT subtypes with 3572 preliminarily promising results $(603,604)$. A recent retrospective report including 200 AIT patients found that the onset of 3574 thyrotoxicosis was significantly earlier in type 1 (median 3.5 months, range 1-61 months) 3575 than type 2 (median 30 months, range 1-95 months), $\mathrm{p}<0.001$ (605). Since $80 \%$ of type 35761 patients in this study had autonomous thyroid nodules or toxic MNG, it is not 3577 unexpected that iodine-induced thyrotoxicosis occurred early in the course of amiodarone 
3578 therapy. However, based on this data, a patient with late-onset of AIT in whom GD has 3579 been excluded is more likely to have type 2 AIT. Another observation reported in this 3580 study is the development of AIT following amiodarone discontinuation. Nineteen 3581 percent of patients (38/200) developed AIT a mean of 5.5 months after the drug was 3582 stopped, 36 of whom had type 2 AIT. Patients who are clinically stable and have definite evidence supporting a distinct 3584 subtype of overt AIT may be tried on appropriate monotherapy. When identified with 3585 certainty, type 1 AIT is best treated with MMI to prevent new hormone synthesis and, 3586 rarely, with added potassium perchlorate $(250 \mathrm{mg}$ four times daily; not available in the 3587 United States) (590). Type 2 AIT is better treated with anti-inflammatory therapy such as 3588 prednisone, with improvement occasionally seen as early as 1 week, and usually within a 3589 few weeks (590). As noted above, some patients with mild type 2 AIT (approximately $359020 \%$ ) resolve spontaneously without stopping amiodarone or administering 3591 corticosteroids $(606,607)$. Most series of patients with AIT contain cases in which sequential therapy for 3593 both subtypes was required before resolution of AIT occurred. These patients are 3594 frequently referred to as "mixed" types of AIT. In a study of 20 patients with AIT that 3595 included both type 1 and type 2 patients, perchlorate was administered alone for 1 month, 3596 resulting in euthyroidism in 12 patients ( 7 with type 1 AIT and 5 with type 2 AIT) (591). 3597 Corticosteroids were then given to the eight nonresponders (including 7 patients with 3598 presumed type 1 disease), and euthyroidism was achieved in all after an average of 3599 approximately 6 weeks (591). Patients are often reclassified retrospectively from type 1 
3600 to type 2 AIT based on a positive response to corticosteroid therapy or after an outcome

3601 of permanent hypothyroidism, both of which would be unlikely in iodine-induced

3602 thyrotoxicosis $(598,606)$. Patients recovering from apparent type 2 AIT should be

3603 monitored for permanent hypothyroidism, which appears to occur more often with AIT

3604 than with subacute thyroiditis (608).

Importantly, individuals with moderate thyrotoxicosis and compromised cardiac

3606 status should be considered for initial combined therapy rather than sequential empiric

3607 therapy. Some centers recommend starting combined therapy with antithyroid drugs and

3608 corticosteroids at the time of initial AIT diagnosis $(594,609)$, and between $16-25 \%$ of

3609 surveyed thyroidologists prefer combination antithyroid drug and corticosteroid therapy

3610 for patients with apparent type 2 AIT (610). A rapid response to combined corticosteroid

3611 and antithyroid drug therapy is believed to favor type 2 AIT (594) and allows a reduction

3612 in antithyroid drugs, although some patients with type 2 AIT have a prolonged course,

3613 particularly those with larger thyroids or worse thyrotoxicosis at the time of diagnosis

3614 (611). A suggested approach to the management of AIT is shown in Figure 1.

3615 Technical remarks: The suggested starting dose of MMI in this setting is $40 \mathrm{mg}$

3616 once daily until the patient is euthyroid (generally 3-6 months). If high doses of MMI

3617 continue to be required, splitting the dose may be more effective. The suggested dose of

3618 corticosteroids in this setting is equivalent to $40 \mathrm{mg}$ prednisone given once daily for $2-4$

3619 weeks, followed by a gradual taper over 2-3 months, based on the patient's clinical

3620 response. 
3622 Patients with AIT who are unresponsive to aggressive medical therapy with MMI and 3623 corticosteroids should undergo thyroidectomy. Strong recommendation, low-quality 3624 evidence.

3625 Patients with AIT who fail to respond to medical therapy should be offered 3626 thyroidectomy before they become excessively debilitated from inadequately controlled 3627 thyrotoxicosis. The patient should be counseled that while thyroidectomy in this setting 3628 carries with it significant morbidity and a high mortality rate (9\%), delay or deferral of 3629 surgery imparts an even higher risk of death (612). Thyroidectomy done under regional 3630 anesthesia when available may be preferred for very ill patients (613). Several surgical 3631 series involving patients with AIT have now been published, with generally favorable 3632 results $(612,614-616)$. Patients in whom amiodarone was stopped during an episode of 3633 AIT should be considered for definitive therapy with RAI or surgery in order to facilitate 3634 reintroduction of amiodarone without concerns about recurrent AIT (617).

\section{[W] How should thyrotoxicosis due to destructive thyroiditis be managed?}

Several varieties of thyroiditis can present with temporary thyrotoxicosis as part

3638 of a classic triphasic course (thyrotoxicosis, hypothyroidism, recovery), including

3639 subacute thyroiditis, painless (silent) thyroiditis, acute (suppurative) thyroiditis, palpation

3640 (traumatic) thyroiditis, postpartum thyroiditis, and drug-induced thyroiditis. In general,

3641 thyroid dysfunction caused by thyroiditis is less severe than that seen with other forms of 3642 endogenous thyrotoxicosis (50); RAIU is universally low during the thyrotoxic stage, 
3643 owing to leaking of preformed thyroid hormone with suppression of serum TSH

3644 concentrations. In this section, subacute, painless, acute and palpation thyroiditis will be

3645 discussed; see section [T4] for a discussion of postpartum and section [X] for a

3646 discussion of drug-induced thyroiditis.

3647 [W1] Subacute thyroiditis

3648 Subacute thyroiditis, also called subacute granulomatous or de Quervain

3649 thyroiditis, is a common cause of thyroid pain (24). The diagnosis of subacute

3650 thyroiditis is based on clinical history, physical examination, laboratory data, and RAIU.

3651 Subacute thyroiditis presents with moderate-to-severe pain in the thyroid, often radiating

3652 to the ears, jaw, or throat. The pain may begin focally and spread from one side to the

3653 other of the gland over several weeks. Patients may have a prodrome of malaise, low-

3654 grade fever, pharyngitis symptoms, and fatigue. The thyroid may be slightly enlarged,

3655 and is firm and painful to palpation. Subacute thyroiditis is thought to be due to a sequela

3656 of an upper respiratory viral infection that involves the thyroid gland.

About $50 \%$ of patients with subacute thyroiditis have an initial thyrotoxic phase

3658 due to unregulated release of preformed thyroid hormone from damaged thyroid

3659 follicular cells (24). Therefore, early in the course of the disease, patients may have

3660 clinical findings of thyrotoxicosis, although this is often mild. The serum TSH level is

3661 suppressed, and the free $\mathrm{T}_{4}$ level may be elevated preferentially to the total $\mathrm{T}_{3}$ level, in

3662 contrast to other endogenous forms of thyrotoxicosis, although there is substantial

3663 overlap among the etiologies (618). In addition to laboratory evidence of thyrotoxicosis,

3664 the erythrocyte sedimentation rate or C-reactive protein (CRP) is elevated, and mild 
anemia and elevation of the white blood count (wbc) are common. Up to $25 \%$ of patients

3666 have low concentrations of anti-thyroid antibodies $(24,619,620)$. RAIU is low, as is

3667 uptake on a thyroid scintigram. Thyroid ultrasonography shows diffuse heterogeneity,

3668 focal hypoechoic areas, and decreased or normal color-flow Doppler, rather than the

3669 enhanced flow characteristic of GD $(621,622)$. A biopsy of the thyroid gland is not

3670 usually necessary in subacute thyroiditis. However, if a biopsy is performed due to

3671 uncertainty of the diagnosis, its result shows granulomatous infiltrate and giant cells,

3672 consistent with a viral infection (24).

The thyrotoxic phase usually lasts 3-6 weeks, ending when the thyroid stores of

3674 preformed hormone are depleted. About $30 \%$ of patients subsequently enter a

3675 hypothyroid phase that can last up to 6 months. Thyroid pain and the elevated ESR have

3676 usually resolved by this time, and the predominant clinical features are those of

3677 hypothyroidism with a small nontender goiter. Most patients become euthyroid again

3678 within 12 months of disease onset, although 5-15\% have persistent hypothyroidism

$3679(24,620,621)$. In addition, recurrence rates of 1-4\% have been reported $(24,620,623)$.

\section{0 - RECOMMENDATION 117}

3681 Patients with mild symptomatic subacute thyroiditis should be treated initially with

3682 beta-adrenergic-blocking drugs and nonsteroidal anti-inflammatory agents.

3683 Corticosteroids should be used instead of nonsteroidal anti-inflammatory agents when

3684 patients fail to respond, or present initially with moderate to severe pain and/or

3685 thyrotoxic symptoms. Strong recommendation, low-quality evidence. 
Subacute thyroiditis is treated with beta-blockers and anti-inflammatory therapy.

3687 Beta-blockers are used as needed to control thyrotoxic symptoms. Nonsteroidal anti-

3688 inflammatory agents (NSAIDs) provide pain relief in patients with mild symptoms, and

3689 should be considered first-line therapy. With NSAIDs, the median time for resolution of

3690 pain is 5 weeks (range 1-20 weeks) (24). Patients who fail to respond to full doses of

3691 NSAIDs over several days should be treated instead with corticosteroid therapy.

3692 Standard recommendations are to use prednisone $40 \mathrm{mg}$ daily for 1-2 weeks followed by

3693 a gradual taper over 2-4 weeks or longer, depending upon clinical response. A

3694 retrospective review found that patients treated with corticosteroids at similar doses had

3695 more rapid resolution of pain (mean duration, 8 days) compared with those treated with

3696 NSAIDs (mean duration, 35 days). However, symptoms can recur as the dose of

3697 corticosteroid is reduced (24). A more recent study reported that a lower initial daily

3698 dose of $15 \mathrm{mg}$ of prednisolone, with tapering by $5 \mathrm{mg}$ every two weeks, was effective.

3699 However, $20 \%$ of patients required longer than 8 weeks to discontinue the glucocorticoid

3700 (624). Levothyroxine may be employed during the hypothyroid stage, but should be

3701 withdrawn after 3-6 months with recovery of normal function verified by thyroid

3702 function testing. ATDs have no role in the treatment of subacute thyroiditis.

$3703 \quad$ [W2] Painless thyroiditis

3704 Painless or silent thyroiditis classically presents with the same triphasic course

3705 described for subacute thyroiditis, but with no prodrome, neck pain, or elevated ESR,

3706 white blood cell count, or CRP (625). The postpartum period is the most common time

3707 when painless thyroiditis is seen, but painless thyroiditis can also occur in nonpregnant 
3708 women and in men. Painless thyroiditis has been described in some types of drug-

3709 induced thyroid dysfunction, including that associated with lithium or cytokine therapy.

3710 Postpartum and drug-induced thyroiditis are discussed in detail in sections [T4] and [X],

3711 respectively. A small nontender goiter is common in all types of painless thyroiditis.

The thyrotoxic phase occurs in 5-20\% of patients and typically lasts 3-4 months.

3713 The hypothyroid phase is more common, or at least is recognized more often, lasting up

3714 to 6 months. Normal thyroid function is reestablished by 12 months in most patients, but

3715 10-20\% have persistent hypothyroidism. Recurrence rates are about 5-10\%, but may be

3716 higher in Japan, with one Japanese study reporting a long-term recurrence rate of $65 \%$

3717 (626). Recurrences are managed in the same manner as the initial occurrence, but rare

3718 patients with multiple recurrences have opted for surgery or RAI ablation of the gland

3719 following recovery from the thyrotoxic phase (626).

Painless thyroiditis is probably an autoimmune disease manifested by positive

3721 anti-TPO antibodies in about $50 \%$ of patients and findings of lymphocytic infiltration on

3722 pathology $(626,627)$. During the thyrotoxic phase, the serum TSH level is suppressed

3723 and free $T_{4}$ levels are elevated, often out of proportion to $T_{3}$ levels. Patients with painless

3724 thyroiditis have a low RAIU and low uptake on a thyroid scintigram during the thyrotoxic

3725 phase, and ultrasound often shows inhomogeneous hypoechogenic texture with decreased

3726 blood flow. These tests and the absence of TRAb antibodies help distinguish painless

3727 thyroiditis from GD (628). 
3729 Patients with symptomatic thyrotoxicosis due to painless thyroiditis should be treated 3730 with beta-adrenergic-blocking drugs to control symptoms. Strong recommendation, $3731 \quad$ low-quality evidence.

3732 Beta-adrenergic blockers can be used to treat thyrotoxic symptoms in patients

3733 with painless thyroiditis, but ATDs have no utility, since new hormone synthesis is

3734 already low in these patients. Rarely, corticosteroids have been used to ameliorate the

3735 severity and the time course of thyrotoxicosis due to painless thyroiditis (629), but they

3736 should be reserved only for more severe cases.

$3737 \quad[$ W3] Acute thyroiditis

\section{- RECOMMENDATION 119}

3739 Acute thyroiditis should be treated with antibiotics and surgical drainage as 3740 determined by clinical judgement. Beta blockers may be used to treat symptoms of 3741 thyrotoxicosis. Strong recommendation, low-quality evidence.

3743 thyroid abscess) are generally euthyroid. However, on occasion, the condition presents as

3744 destructive thyroiditis with thyrotoxicosis (630). Ultrasound or CT examinations are

3745 usually diagnostic, showing hypoechoic lesions in and around the affected thyroid lobe,

3746 destruction of the lobe, and abscess formation. However, early in the process, radiologic

3747 examination may be nonspecific, often leading to the erroneous diagnoses of subacute

3748 thyroiditis (631). The etiology of acute thyroiditis is most frequently a bacterial infection

3749 affecting the thyroid, either through hematogenous spread or direct extension through a 
3750 fistula from an infected pyriform sinus. Therapy involves systemic antibiotics as well as

3751 abscess drainage or removal, and excision or occlusion of the offending pyriform sinus.

3752 Thyrotoxicosis should be treated symptomatically with beta-blocking agents. As in other

3753 forms of destructive thyroiditis, there is no role for ATDs.

$3754 \quad$ [W4] Palpation thyroiditis

In 1975, Carney et al described a nonspecific multifocal granulomatous folliculitis

3756 in thyroid glands removed at surgery for thyroid-related or unrelated conditions (632).

3757 They named this pathologic entity "palpation thyroiditis," concluding that it was due to

3758 palpation of the thyroid gland at surgery. It was generally thought to be of little clinical

3759 importance, except for rare case reports of patients who developed thyrotoxicosis

3760 following manipulation of the thyroid gland during surgery (633-636). However, a recent

3761 study suggested that the rate of transient overt or subclinical thyrotoxicosis following

3762 parathyroid surgery may be as high as $30 \%$, although there was likely ascertainment bias

3763 because not all patients had post-operative TSH levels measured (637). There are no data

3764 on treatment of palpation thyroiditis, although the use of beta blockers for symptomatic

3765 thyrotoxicosis seems reasonable.

3767 [X] How should other causes of thyrotoxicosis be managed?

3768 Tables 16 and 17 summarize drug-associated and unusual causes of thyrotoxicosis. 
3770 Patients taking medications known to cause thyrotoxicosis, including interferon- $\alpha$, IL-

37712 2, tyrosine kinase inhibitors, and lithium, should be monitored clinically and

3772 biochemically at 6-month intervals for the development of thyroid dysfunction.

3773 Patients who develop thyrotoxicosis should be evaluated to determine etiology and

3774 treated accordingly. Strong recommendation, low-quality evidence.

$3775 \quad[\mathrm{X} 1]$ Interferon- $\alpha$ and interleukin-2

Interferon- $\alpha(\mathrm{IFN}-\alpha)$ and interleukin-2-treated patients are at increased risk for

3777 developing thyrotoxicosis, especially those with pre-existing thyroid autoimmunity. A

3778 recent study including 1233 patients who were euthyroid at baseline found that $79(6.4 \%)$

3779 patients developed a biphasic thyroiditis and an additional $57(4.6 \%)$ patients developed a

3780 suppressed TSH value (638). The latter group included 33 patients with mild TSH

3781 suppression and 24 with a TSH value $<0.1 \mathrm{mU} / \mathrm{L}$, among whom 11 had free $\mathrm{T}_{4}$ elevation

3782 and 5 required antithyroid drug therapy (638). Thyrotoxicosis in patients treated with

3783 IFN- $\alpha$ can be due to either painless thyroiditis or GD (639). In a review of published

3784 cases from 8 series, $69 \%$ of 49 patients with IFN- $\alpha$-associated thyrotoxicosis, for whom

3785 an etiology was identified, were found to have GD, based on either positive TRAb titers

3786 or requirement for a prolonged course of ATDs (640). An earlier literature review found

3787 that $2.4 \%$ of 1664 patients treated with IFN- $\alpha$ therapy for hepatitis $\mathrm{C}$ infection developed

3788 thyrotoxicosis, although the specific etiology was not consistently identified (641).

$3789 \quad$ [X2] Tyrosine Kinase Inhibitors 
3791 nilotinib (651) have each been associated with a transient thyrotoxicosis due to

3792 destructive thyroiditis. One study of 69 patients treated with sorafenib for metastatic

3793 renal cell carcinoma found that $11(16 \%)$ developed transient thyroiditis followed by

3794 hypothyroidism (649). Another study found that 6 of 31 (19.3\%) receiving sunitinib

3795 therapy for metastatic renal cell carcinoma developed thyrotoxicosis, including one case

3796 of thyroid storm (644).

[X3] Lithium

Patients taking lithium for bipolar disorder are at a high risk of developing thyroid

3799 dysfunction, including both hypothyroidism, and to a lesser extent, thyrotoxicosis (652).

3800 Two published series have identified the development of thyrotoxicosis in $0.6 \%$ and $3.0 \%$

3801 of patients, respectively $(653,654)$. An epidemiological study of hyperthyroidism

3802 occurring over a 3-year period in Denmark identified lithium-associated thyrotoxicosis as

3803 the etiology in $0.7 \%$ of all cases (432). A case series of 24 patients with lithium-

3804 associated thyrotoxicosis identified GD in $12(50 \%)$, painless thyroiditis in two patients,

3805 toxic multinodular goiter in 3 patients, and no identified etiology in 7 patients (655).

3806 Another more recent series found that $1.4 \%$ of referrals to a thyroid clinic over a 12 -year

3807 period were for lithium related thyrotoxicosis (656). Patients in this series had been

3808 taking lithium for a median duration of 6 years (range $0.6-25$ years), and $87 \%$ were

3809 women. Diagnostic evaluation found that $11(47.8 \%)$ had GD, $9(39 \%)$ had painless

3810 thyroiditis, 2 had toxic MNG, and one patient had subacute thyroiditis (656). A smaller

3811 series described three cases of GD occurring in patients receiving lithium (657). In a 
3812 retrospective review of 100 cases of thyroiditis and 400 cases of GD occurring at a single

3813 medical center, 6 cases of painless thyroiditis had a history of recent lithium exposure,

3814 representing a nearly 5-fold increase compared to cases of lithium exposure in patients

3815 with GD (19).

$3816 \quad$ [X4] TSH-secreting pituitary tumors

3817 - RECOMMENDATION 121

3818 The diagnosis of TSH-secreting pituitary tumor should be based on an inappropriately

3819 normal or elevated serum TSH level associated with elevated free $\mathrm{T}_{4}$ and total $\mathrm{T}_{3}$

3820 concentrations, generally associated with a pituitary tumor on MRI or CT, and the

3821 absence of a family history or genetic testing consistent with thyroid hormone

3822 resistance. Strong recommendation, low-quality evidence.

TSH-secreting pituitary adenomas are rare tumors and represent an even rarer

3824 cause of hyperthyroidism. Recent data from the Swedish registry reported an incidence of

38250.15 per 1 million inhabitants with a prevalence of 2.8 cases per million (658). After

3826 excluding laboratory interference with either the free $\mathrm{T}_{4}$ or TSH assay, as may occur with

$3827 \mathrm{~T}_{4}$ antibodies and heterophilic antibodies, respectively, distinction between a TSH-

3828 secreting adenoma and thyroid hormone resistance is important, since thyroid function

3829 test results are similar, yet management is quite different for these two disorders. TSH-

3830 secreting adenomas are more likely to have concurrent alpha-subunit elevation (not

3831 useful in postmenopausal women due to concurrent gonadotropin elevation), a blunted

3832 TSH response to thyrotropin-releasing hormone (TRH) (when available), elevated sex- 
3833 hormone-binding globulin and resting energy expenditure, and clinical evidence of

3834 thyrotoxicosis, as well as an anatomic abnormality on MRI of the pituitary. Finally, a

3835 response to somatostatin analog therapy with clinical improvement lends support to the

3836 diagnosis of a TSH adenoma in cases in which diagnostic uncertainty persists. Although

3837 most TSH-secreting pituitary adenomas only secrete TSH, co-secretion of prolactin or

3838 growth hormone is possible and should be assessed concurrently along with assessment

3839 of the pituitray-adrenal axis and pituitary-gonadal axes.

Technical remarks: Genetic testing for thyroid hormone resistance is

3841 commercially available and may be useful in equivocal cases, especially in those patients

3842 without family members available for thyroid function testing. Calculation of the molar

3843 alpha subunit/TSH ratio can be accomplished by dividing the alpha subunit concentration

3844 (ng/mL) by TSH (mU/L) and multiplying by 10. A ratio greater than 1 favors a TSH-

3845 secreting pituitary adenoma.

Pituitary surgery is generally the mainstay of therapy for TSH-producing pituitary

3847 tumors. In a recent series of 68 patients undergoing transsphenoidal surgery, $75 \%$

3848 normalize thyroid function after surgery, 58\% normalized both pituitary imaging and

3849 TSH hypersecretion, 9\% developed new deficiencies, and 3\% experienced tumor

3850 recurrence (659). The patient should be made euthyroid preoperatively. Long-term ATD

3851 therapy and other measures directed at the thyroid, such as RAI or thyroidectomy, are

3852 generally avoided due to theoretical concerns of tumor growth. Preoperative adjunctive

3853 therapy with octreotide and dopamine agonist therapy has been examined. Treatment

3854 with octreotide results in a $>50 \%$ reduction in serum TSH values in the majority of 
3855 patients treated, and a concurrent return to euthyroidism in most (43). A reduction in

3856 tumor size has been observed in $20 \%-50 \%$ of patients treated with octreotide $(43,660)$,

3857 but less impressive results have been obtained with bromocriptine therapy (660).

3858 However, presurgical medical treatment did not significantly improve surgical outcome

3859 (63\% vs $57 \%$ had negative tumor imaging after surgery) (659). In a recent case series of

38607 patients treated with octreotide without prior surgery, mean free $\mathrm{T}_{4}$ and $\mathrm{T}_{3}$ levels were

3861 reduced by nearly $50 \%$ in the first three months of therapy and 6 of 7 patients

3862 experienced tumor volume reduction (661).

3863 Sterotactic or conventional radiotherapy has also been used in cases that prove

3864 refractory to medical therapy. Radiotherapy controlled thyroid hypersecretion in $37 \%$ of

3865 patients treated with this modality, but hypopituitarism occurred in $32 \%$ of those treated

3866 (659). For patients with TSH-producing adenomas who are considered poor surgical

3867 candidates, primary medical therapy with octreotide can be considered (661). Patients

3868 who fail to respond to pituitary surgery and somatostatin analog therapy or have tumor

3869 enlargement despite these measures are sometimes treated with radiation therapy.

\section{- RECOMMENDATION 122}

3871 Patients with TSH-secreting pituitary adenomas should undergo surgery performed by

3872 an experienced pituitary surgeon. Strong recommendation, low-quality evidence.

3873 Technical remarks: Postoperative adjunctive therapy with octreotide and/or

3874 external beam radiation therapy may be useful in managing patients with persistent 
$3877 \quad[X 5]$ Struma ovarii

\section{- RECOMMENDATION 123}

Patients with struma ovarii should be treated initially with surgical resection following

3880 preoperative normalization of thyroid hormones. Strong recommendation, low-

$3881 \quad$ quality evidence.

3883 component of an ovarian tumor, is quite rare, representing $<1 \%$ of all ovarian tumors.

3884 Approximately 5-10\% of patients with struma ovarii present with thyrotoxicosis (662)

3885 due to either autonomous ectopic thyroid function or the coexistence of GD, and up to

$388625 \%$ of struma ovarii tumors contain elements of papillary thyroid cancer. Patients

3887 previously treated for GD may have persistent or recurrent hyperthyroidism due to the

3888 action of TRAb on the ectopic thyroid tissue (663). The diagnosis should be considered

3889 in any thyrotoxic patient with a very low or absent RAI uptake over the eutopic thyroid

3890 gland. Other conditions that present with this constellation of findings include various

3891 forms of thyroiditis, factitious thyrotoxicosis, and iodine-induced hyperthyroidism. In

3892 struma ovarii, the RAI is concentrated in the pelvic region over the teratoma.

3893 Cosynchronous primary thyroid cancer occurred in $9 \%$ of patients in one series of 68

3894 patients identified in the SEER database (664). Treatment of struma ovarii generally

3895 involves surgical removal, performed both to cure the hyperthyroidism and to eliminate 
3896 the risk of untreated ectopic thyroid cancer. Preoperative treatment with beta-adrenergic-

3897 blocking agents and ATDs is warranted to restore euthyroidism before surgery.

3899 is generally given following surgical removal of both the ovarian tumor and the patient's

3900 thyroid to facilitate delivery of isotope to any potential residual malignant cells.

$3901 \quad[X 6]$ Choriocarcinoma

3903 Treatment of hyperthyroidism due to choriocarcinoma should include both MMI and

3904 treatment directed against the primary tumor. Strong recommendation, low-quality

3905 evidence.

3906 Patients with choriocarcinoma, including molar pregnancy and testicular cancer,

3907 may present with thyrotoxicosis due to the effect of tumor-derived hCG upon the TSH

3908 receptor (665-668). This cross-stimulation only occurs at very high levels of hCG, since

3909 hCG is only a weak agonist for the TSH receptor. Therefore, patients with testicular

3910 choriocarcinoma presenting with overt thyrotoxicosis may have widely metastatic disease

3911 at presentation $(667,668)$. Treatment of hyperthyroidism due to choriocarcinoma involves

3912 both treatment directed against the primary tumor and ATDs.

$3913 \quad[X 7]$ Thyrotoxicosis factitia

3914 Thyrotoxicosis factitia includes all causes of thyrotoxicosis due to the ingestion of 3915 thyroid hormone. This may include intentional ingestion of thyroid hormone either 
surreptitiously or iatrogenically, as well as unintentional ingestion either accidentally,

3917 such as in pediatric poisoning or pharmacy error, or through ingestion of supplements

3918 that contain thyroid hormone (669). Historically, accidental thyroid hormone ingestion

3919 has occurred as a result of eating meat contaminated with animal thyroid tissue

3920 ("hamburger thyrotoxicosis") (670).

Whereas iatrogenic causes of thyrotoxicosis factitia are easily identified,

3922 surreptitious use of thyroid hormone may present a diagnostic quandary. Clues to this

3923 diagnosis are an absence of goiter, a suppressed serum thyroglobulin level, and a

3924 decreased RAI uptake. In a patient who has circulating antithyroglobulin antibodies that

3925 artifactually render the serum thyroglobulin undetectable in immunometric assays, the

3926 distinction between painless thyroiditis and factitious thyrotoxicosis can be difficult. In

3927 both situations there will be elevated levels of $T_{4}$, a high $T_{4} / T_{3}$ ratio (with exogenous

3928 levothyroxine), a small thyroid, and a low thyroidal RAI uptake. Thyroid ultrasound may

3929 be helpful, as the thyroid has a heterogeneous echotexture and is normal-sized or slightly

3930 enlarged in painless thyroiditis, while it is small with a normal echotexture in an

3931 otherwise normal individual who is ingesting thyroid hormone surreptitiously. Fecal

3932 levothyroxine has been measured as a means of distinguishing surreptitious use of thyroid

3933 hormone from painless thyroiditis (54). A disproportionately elevated $\mathrm{T}_{3}$ level suggests

3934 that the patient may be ingesting liothyronine or a combination $\mathrm{T}_{4} / \mathrm{T}_{3}$ preparation.

Severe thyrotoxicosis and rarely, thyroid storm, have been reported following

3936 thyroid hormone overdose or poisoning. Treatment with Cholestyramine (671) and

3937 charcoal hemoperfusion (672) have been used in this circumstance. 
3940 been described in a handful of cases. Typically, patients have either a very large primary

3941 follicular cancer or widely metastatic follicular thyroid cancer, and may have coexisting

3942 TRAb as the proximate cause of the thyrotoxicosis (673). In general, functioning

3943 metastasis are treated with RAI with the addition of ATDs as needed for persistent

3944 hyperthyroidism. Recombinant human TSH should be avoided in these patients. Patients

3945 with massive metastatic FC may also exhibit $\mathrm{T}_{3}$ thyrotoxicosis, most likely due to

3946 increased conversion of $\mathrm{T}_{4}$ to $\mathrm{T}_{3}$ by tumor expressing high type 1 and type 2 deiodinase

3947 activities (674). Thus, occasional measurement of serum $\mathrm{T}_{3}$ in addition to $\mathrm{FT}_{4}$ and $\mathrm{TSH}$ is

3948 recommended in patients with a large metastatic tumor burden, particularly if $\mathrm{FT}_{4}$

3949 decreases on fixed doses of levothyroxine. 